\title{
Nonlinear dynamics of semiconductor lasers with active optical feedback
}

\author{
D I S S E R T A T I O N \\ zur Erlangung des akademischen Grades \\ doctor rerum naturalium \\ (Dr. rer. nat.) \\ im Fach Physik \\ eingereicht an der \\ Mathematisch-Naturwissenschaftlichen Fakultät I \\ Humboldt-Universität zu Berlin \\ von \\ Herr Dipl.-Phys. Stefan Bauer \\ geboren am 24.01.1973 in Vilshofen
}

Präsident der Humboldt-Universität zu Berlin:

Prof. Dr. Jürgen Mlynek

Dekan der Mathematisch-Naturwissenschaftlichen Fakultät I:

Prof. Thomas Buckhout, PhD

Gutachter:

1. Prof. Dr. Fritz Henneberger

2. Prof. Dr. Oliver Benson

3. Prof. Dr. Wolfgang Elsäßer

eingereicht am: $\quad 01.07 .2004$

Tag der mündlichen Prüfung: $\quad$ 11.01.2005 



\section{Contents}

1 Introduction 1

2 Distributed feedback laser $\quad 5$

2.1 Theoretical description of the DFB laser . . . . . . . . . . . . 5

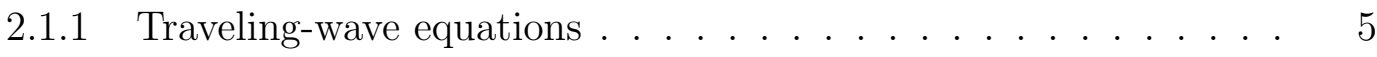

2.1.2 Dynamics of optical modes . . . . . . . . . . . . . . . . 10

2.1.3 Simulation tool LDSL . . . . . . . . . . . . . . . . . . . 11

2.2 Experimental characterization . . . . . . . . . . . . . . . 12

2.2.1 Design of investigated lasers . . . . . . . . . . . . . . 12

2.2.2 Parameters of investigated laser structure . . . . . . . . . . . 13

2.2.3 Single-mode operation due to LSHB . . . . . . . . . . . . . . 15

2.2.4 Solitary DFB laser dynamics . . . . . . . . . . . . . . . . 17

3 Delayed optical feedback $\quad 19$

3.1 Theoretical predictions . . . . . . . . . . . . . . . . . . . . . 19

3.1.1 Modes of DFB laser with feedback . . . . . . . . . . . 20

3.1.2 Folding of modes . . . . . . . . . . . . . . . . . . . 21

3.1 .3 Feedback regimes . . . . . . . . . . . . . . . . 23

3.1.4 Characteristic dynamics in the very short cavity limit . . . . . . 24

3.2 Experimental verification: the passive feedback laser . . . . . . . . . . . 27

$3.2 .1 \quad$ Device . . . . . . . . . . . . . . . . . . 27

3.2 .2 Overview on dynamics . . . . . . . . . . . . . . . . 28

4 The concept of active feedback lasers $\quad 30$

4.1 Idea and realization . . . . . . . . . . . . . . . . . 30

4.2 Integrated feedback cavity characteristics . . . . . . . . . . . . . . 32

4.3 Optical modes: Impact of strong feedback . . . . . . . . . . . . . . . . 34

4.4 Experimental exploration of device dynamics . . . . . . . . . . . . . . . . . 35

4.4 .1 Measurement setup . . . . . . . . . . . . . . 35

4.4 .2 Overview ............................ 37

4.5 Numerical simulation results . . . . . . . . . . . . . . . . . . . . . . 39

5 Bifurcations of the AFL: theoretical predictions 44

5.1 Bifurcation diagram . . . . . . . . . . . . . . . . . . . . . 44 
5.2 Folds of stationary states . . . . . . . . . . . . . . . . . . . 46

5.3 Hopf bifurcations . . . . . . . . . . . . . . . . . . . . 47

5.4 Bifurcations of limit cycles . . . . . . . . . . . . . . . . . . . . . . . 48

5.4.1 Homoclinic bifurcation . . . . . . . . . . . . . . . . 48

5.4.2 Saddle-node of limit cycles . . . . . . . . . . . . . . . . . . . . 48

5.4.3 Period doubling . . . . . . . . . . . . . . . . . . . . . . 49

5.4.4 Torus bifurcation ....................... 49

$6 \quad$ Experimental verification of bifurcations $\quad 51$

6.1 Detailed overview on device dynamics . . . . . . . . . . . . . . 51

6.2 Hysteresis . . . . . . . . . . . . . . . . . . . . 52

6.3 Hopf bifurcations . . . . . . . . . . . . . . . . 56

6.4 Homoclinic bifurcation . . . . . . . . . . . . . . . . . . . . . 59

6.5 Torus bifurcation . . . . . . . . . . . . . . . . . . 59

7 The torus regime $\quad 61$

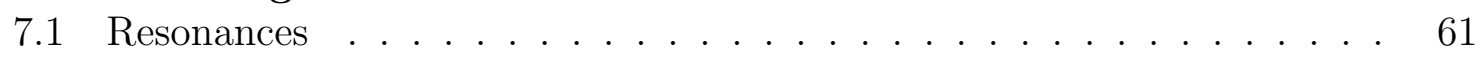

7.2 Break-up of torus . . . . . . . . . . . . . . . . . 67

7.3 Boundary crisis . . . . . . . . . . . . . . . . . . . . 69

8 Impact of noise $\quad \mathbf{7 2}$

8.1 Noisy precursors . . . . . . . . . . . . . . . . . . . . 72

8.2 Noise induced irregular dynamics . . . . . . . . . . . . . . . 74

9 Locking of AFL dynamics $\quad 77$

9.1 Locking of regular pulsations . . . . . . . . . . . . . . . . 77

9.2 Superharmonic locking of PD . . . . . . . . . . . . . . 80

10 Summary and Outlook $\quad 82$

A Appendix $\quad \mathbf{8 5}$

A.1 Device parameter used for simulation . . . . . . . . . . . 85

A.2 List of Abbreviations . . . . . . . . . . . . . . . . . 86

A.3 Modifications of TWE-model . . . . . . . . . . . . . . . 87

A.4 Stability of AFL dynamics . . . . . . . . . . . . . . . . . . 89 


\begin{abstract}
In this work, dynamical effects in semiconductor lasers due to active optical feedback are investigated. A novel laser type based on distributed feedback (DFB) structures is developed and realized. Experiments and simulations give a comprehensive overview on the nonlinear dynamics of this laser.

Based on the coupled-mode theory the basic characteristics of DFB lasers are introduced. Experiments show that controlled longitudinal spatial hole burning allows for stable singlemode lasing.

The impact of delayed feedback on the static mode structure is discussed. An important phenomenon is the folding of modes, which induces the coexistence of several laser states and gives rise to hysteresis effects. In addition to a modified stationary behavior, delayed optical feedback can cause unstable laser output. Depending on feedback delay and strength different dynamics is observed. The regime of interest is the non-zero short delay limit. Here, undamped relaxation oscillations and a beating of distinct compound-cavity modes are the characteristic instabilities. These oscillation types are experimentally confirmed by a passive feedback laser.

Since the feedback strength provided by a passive delay section is limited and lacks efficient control an improved laser type is developed: the active feedback laser (AFL). By the integration of separate phase tuning and amplifier sections, feedback strength and phase become adjustable.

Simulations and a complete bifurcation analysis are presented for the AFL. They demonstrate that this laser provides access to the full spectrum of laser dynamics in the very short delay limit. A detailed analysis of the device with respect to the most important bifurcation types is given. The hysteresis behavior is characterized. Supercritical Hopf bifurcations are identified to be responsible for the onset of both pulsation types. Since mode beating and relaxation oscillations can coexist in a large parameter range, characteristic phenomena of interacting nonlinear oscillators are observed. Resonant states are examined; a reduced spectral linewidth and a decoupling of oscillator frequencies from the control parameters occur. Furthermore, the coexistence of oscillations gives rise to the break-up of regular pulsations into chaotic emission. Simulations are employed to show the existence of a boundary crisis scenario.

The modification of the laser dynamics by the impact of spontaneous emission noise is shown with the example of noisy precursors. Irregular dynamics which is attributed to noise can be observed at the transition from undamped relaxation oscillations to beating pulsations. Finally, the applicability of the AFL is demonstrated by the locking to optical data signals with a data rate of $40 \mathrm{Gbit} / \mathrm{s}$. Operation points with coexisting oscillations are shown to be suited for all-optical frequency division.
\end{abstract}

\title{
Keywords:
}

semiconductor laser, nonlinear dynamics, optical feedback, bifurcation analysis 



\section{Zusammenfassung}

In dieser Arbeit werden dynamische Effekte in Halbleiterlasern aufgrund aktiver optischer Rückkopplung mit kurzer Verzögerungsstrecke untersucht. Auf der Basis von Laserstrukturen mit verteilter Rückkopplung wird ein neuartiger Lasertyp entwickelt und realisiert. Anhand von Experimenten und Simulationen wird ein umfassender Überblick der nichtlinearen Dynamik dieses Lasers gegeben.

Die grundlegenden Eigenschaften von Lasern mit verteilter Rückkopplung werden mittels der Theorie gekoppelter Moden diskutiert. Experimente belegen, dass kontrolliertes räumliches Lochbrennen eine stabil einmodige Laseremission ermöglicht.

Der Einfluss verzögerter Rückkopplung auf die statische Modenstruktur wird untersucht. Ein grundlegendes Phänomen ist die Faltung der Moden, wodurch mehrere Laserzustände koexistieren können und Hysterese verursachen. Neben einem modifizierten statischen Verhalten kann die verzögerte Rückkopplung Instabilitäten der Laseremission hervorrufen. In Abhängigkeit von der Verzögerungszeit und der Rückkoppelstärke entsteht unterschiedliche Dynamik. Schwerpunkt dieser Arbeit ist der Bereich nichtverschwindender, kurzer Rückkoppelverzögerung. Hier werden entdämpfte Relaxationsoszillationen und Schwebungsphänomene longitudinaler Kavitätsmoden beobachtet. Ein Laser mit kurzer passiver Rückkoppelkavität dient zur Verifikation dieser grundlegenden Instabilitäten.

Weil die Rückkoppelstärke einer passiven Verzögerungsstrecke durch Verluste begrenzt ist und nur unzureichend kontrolliert werden kann, wird in der vorliegenden Arbeit ein neuer Lasertyp mit aktivem optischen Rückkoppelweg entwickelt. Eine Phasensteuersektion und ein zusätzlicher Verstärker ermöglichen die Steuerung von Rückkoppelphase und -intensität.

Für dieses Bauelement werden Simulationen und eine umfassende Bifurkationsanalyse durchgeführt. Es zeigt sich, dass dieser Laser das ganze Spektrum nichtlinearer Dynamik im Bereich sehr kurzer Verzögerungszeiten abdeckt. Die wichtigsten Bifurkationstypen werden eingehend experimentell untersucht. Hierzu gehört das Hystereseverhalten. Superkritische Hopf-Bifurkationen werden als Ursache für entdämpfte Relaxationsoszillationen und Schwebungspulsationen identifiziert. Da diese Pulsationstypen in weiten Bereichen koexistieren, können Phänomene wechselwirkender, nichtlinearer Oszillatoren untersucht werden. Insbesondere werden resonante Zustände beobachtet. Sie zeichnen sich durch verringerte spektrale Linienbreiten und die Entkopplung der Pulsationsfrequenz von den LaserKontrollparametern aus. Darüber hinaus kann in diesem Bereich auch der Übergang von regulären Pulsationen zu chaotischem Verhalten beobachtet werden. Mit Hilfe von Simulationen wird die Kollision eines Attraktors mit dem Rand seines Einzugsgebiets (boundary crisis) nachgewiesen.

Der Einfluss von spontaner Emission auf die Laserdynamik zeigt sich durch rauschinduzierte Vorläufer von Bifurkationen. Im Übergangsbereich von entdämpften Relaxationsoszillationen zu Schwebungspulsationen verursacht Rauschen irreguläre Dynamik. Abschliessend wird die praktische Anwendbarkeit von Lasern mit aktiver optischer Rückkopplung mit Synchronisationsversuchen bei einer Datenrate von $40 \mathrm{Gbit} / \mathrm{s}$ verifiziert. Im Bereich koexistierender Oszillationen kann das interne Synchronisationsverhalten für die rein optische Frequenzteilung benutzt werden.

\section{Schlagwörter:}

Halbleiterlaser, nichtlineare Dynamik, optische Rückkopplung, Bifurkationsanalyse 



\section{Publications related to this thesis}

\section{Journals}

1. C. Bornholdt, B. Sartorius, S. Schelhase, M. Möhrle, and S. Bauer: "Self-pulsating DFB laser for all-optical clock recovery at 40 Gbit/s", Electronics Letters, vol. 36, no. 4, 327-328, 2000.

2. G. Grosskopf, D. Rohde, R. Eggemann, S. Bauer, C. Bornholdt, M. Möhrle, and B. Sartorius: "Optical millimeter-wave generation and wireless data transmission using a dual-mode laser", IEEE Photon. Tech. Lett., vol. 12, 1692-1694, 2000.

3. M. Möhrle, B. Sartorius, C. Bornholdt, S. Bauer, O. Brox, A. Sigmund, R. Steingrüber, M. Radziunas, and H.-J. Wünsche: "Detuned Grating Multisection-RWDFB Lasers for High Speed Optical Signal Processing", IEEE J. on Sel. Top. Quantum Electron., vol. 7, 217-223, 2001.

4. S. Bauer, O. Brox, J. Kreissl, G. Sahin, and B. Sartorius: "Optical Microwave Source", Electron. Lett., vol. 38, 334-335, 2002.

5. H.-J. Wünsche, M. Radziunas, S. Bauer, O. Brox, and B. Sartorius: "Modeling of Mode Control and Noise in Self-Pulsating PhaseCOMB Lasers", IEEE J. on Sel. Top. Quantum Electron., vol. 9, no. 3, 857-864, 2003.

6. O. Brox, S. Bauer, M. Radziunas, M. Wolfrum, J. Sieber, J. Kreissl, B. Sartorius, and H.-J. Wünsche: "Tunable High-Frequency Generation in DFB-Lasers with Amplified Feedback", IEEE J. Quantum Electron., vol. 39, no. 11, 1381-1387, 2003.

7. S. Bauer, O. Brox, J. Kreissl, B. Sartorius, M. Radziunas, J. Sieber, H.-J. Wünsche, and F. Henneberger: "Nonlinear dynamics of semiconductor lasers with active optical feedback", Phys. Rev. E, vol. 69, no. 1, 016206, 2004.

8. O. Ushakov, S. Bauer, O. Brox, H.-J. Wünsche, and F. Henneberger: "Self-Organization in Semiconductor Lasers with Ultra-Short Optical Feedback", Phys. Rev. Lett., vol. 92, no. 4, 043902, 2004.

9. S. Bauer, O. Brox, J. Kreissl, B. Sartorius, H.-J. Wünsche, and F. Henneberger: "Synchronization in Active Feedback Lasers", in preparation.

\section{Patents}

B. Sartorius, S. Bauer, C. Bornholdt, O. Brox: "Method for Controlling a Laser Based 3R Regenerator", WO 02/084901, 24.10.2002.

S. Bauer, O. Brox: "Optische Mikrowellenquelle", PCT/DE 02/04458, 29.11.2002. 


\section{Contributions to Conferences}

1. S. Bauer, O. Brox, D. Hoffmann, M. Möhrle, G. Sahin, B. Sartorius, S. Schelhase, B. Lavigne, and D. Chiaroni: "Ultrafast locking optical clock for IP packet switching applications", In Proceedings of OFC2000, Baltimore, USA, TuF5.1, 2000.

2. B. Sartorius, C. Bornholdt, S. Bauer, M. Möhrle, P. Brindel, and O. Leclerc: "System application of $40 \mathrm{GHz}$ all-optical clock in a $40 \mathrm{Gbit} / \mathrm{s}$ optical 3R regenerator", In Proceedings of OFC 2000, Baltimore, USA, vol. 4, 199-201, 2000.

3. M. Möhrle, B. Sartorius, C. Bornholdt, S. Bauer, O. Brox, A. Sigmund, R. Steingrüber, M. Radziunas, and H.-J. Wünsche: "Detuned grating multi-sectionRW-DFB-lasers for high speed optical signal processing", In Proceedings of the Semiconductor Laser Conference 2000, Monterey, USA, 143-144, 2000.

4. O. Brox, S. Bauer, C. Bornholdt, D. Hoffmann, M. Möhrle, G. Sahin, and B. Sartorius: "Optical 3R regenerator based on Q-switched laser", In Proceedings of OFC 2001, Anaheim, USA, MG6.1, 2001.

5. S. Bauer, O. Brox, C. Bornholdt, B. Sartorius, and M. Möhrle: "Laser Based 3R Regeneration for High-Speed All-Optical Networks", In Proceedings of WAON 2001, Zagreb, Croatia, 59-62, 2001.

6. B. Sartorius, C. Bornholdt, S. Bauer, and M. Möhrle: "40 GHz optical clock recovery for application in asynchronous networks", In Proceedings of ECOC 2001, Amsterdam, Netherlands, vol. 3, 442-443, 2001.

7. C. Bornholdt, S. Bauer, M. Möhrle, H.-P. Nolting, and B. Sartorius: "All-optical clock recovery at $80 \mathrm{GHz}$ and beyond", In Proceedings of ECOC 2001, Amsterdam, Netherlands, vol. 4, 502-503, 2001.

8. B. Sartorius, S. Bauer, C. Bornholdt, M. Möhrle, H.-P. Nolting, and J. Slovak: "All-optical clock recovery for signal processing and regeneration", In Proceedings of IEEE/LEOS Summer Topical Meetings 2002, Mont Tremblant, Canada, TuO1.29, 2002.

9. M. Möhrle, C. Bornholdt, O. Brox, S. Bauer and B. Sartorius: "Multi-section DFB lasers for high speed signal processing/regeneration", In Proceedings of OFC 2002, Anaheim, USA, 136-138, 2002.

10. S. Bauer, O. Brox, J. Sieber, and M. Wolfrum: "Novel Concept for a Tunable Optical Microwave Source", In Proceedings of OFC 2002, Anaheim, USA, vol. 1, 478-479, 2002. 
11. O. Brox, S. Bauer, J. Kreissl, M. Möhrle, B. Sartorius, and H.-J. Wünsche: "DFB-Lasers with Amplified Optical Feedback for Tunable High-Frequency Generation", In Technical Digest Optical Amplifiers and their Applications, OAA 2002, Vancouver, Canada, OWC2, 2002.

12. S. Bauer, O. Brox, C. Bornholdt, J. Slovak, J. Kreissl, and B. Sartorius: "SelfPulsating Multi-Section DFB Lasers and Their Applications", In Proceedings of IEEE LEOS 2003 Annual Meeting, Tucson, Arizona, invited talk, vol. 1, 45-46, 2003.

13. O. Brox, S. Bauer, C. Bobbert, G. Bramann, J. Kreissl, B. Sartorius, M. Schmidt, K. Schuh, B. Junginger, and E. Lach: "160 to $40 \mathrm{~Gb} / \mathrm{s}$ Demultiplexing Using a Self-Pulsating Laser Based Clock Recovery", In Proceedings of OFC 2003, Georgia, Atlanta, vol. 1, 105-106, 2003.

14. S. Bauer, O. Brox, M. Biletzke, J. Kreissl, M. Radziunas, B. Sartorius, and H.-J. Wünsche: "Speed Potential of Active Feedback Lasers", In Proceedings of CLEOe 2003, Munich, Germany, CC1M, 2003.

15. O. Brox, S. Bauer, C. Bobbert, M. Biletzke, J. Kreissl, H.-J. Wünsche, and B. Sartorius: "Timing Stability of Injection-Locked $40 \mathrm{GHz}$ Self-Pulsating DFBLasers", In Proceedings of ECOC 2003, Rimini, Italy, Tu4.5.3, 2003.

16. J. Kreissl, S. Bauer, O. Brox, E. Lenz, T. Gärtner, M. Biletzke, B. Sartorius, and H.-P. Nolting: "Self-Pulsating Lasers with complex-coupled DFBs", In Proceedings of ECOC 2003, Rimini, Italy, Tu4.5.2, 2003.

17. O. Brox, S. Bauer, M. Biletzke, H. Ding, J. Kreissl, H.-J. Wünsche, and B. Sartorius: "Short Feedback DFB for $40 \mathrm{GHz}$ Clock Recovery: Noise Induced Intensity Fluctuations and Jitter", In Proceedings of OFC 2004, Los Angeles, USA, MF55, 2004.

18. S. Bauer, B. Hüttl, O. Brox, M. Biletzke, J. Kreissl, B. Sartorius, and H.-J. Wünsche: "Unifying mode-locking and self-pulsations in a laser with active feedback", In Proceedings of OAA 2004, San Francisco, USA, OTuD2, 2004.

19. A. Siahlo, A. T. Clausen, J. Seoane, L. K. Oxenløwe, K. S. Berg, P. Jeppesen, O. Brox, S. Bauer, J. Kreissl, and B. Sartorius: "Pulse Compression of a $40 \mathrm{GHz}$ DFB based Optical Clock Recovery", In Proceedings of ECOC 2004, Stockholm, Sweden, We2.5.4, 2004. 



\section{Chapter 1}

\section{Introduction}

Optical technologies have become a part of everyday life. They revolutionized many economic fields, from telecommunication and life sciences to the consumer market, e.g. with the omnipresent CD-players. A key device for optical technologies is the semiconductor laser (SL) due to its compactness, high efficiency and reliability.

From the first demonstration of a SL in 1962 by Hall et al. [43] to a market volume of 6.5 billion euro in the year 2000 [44] many technological problems had to be solved. Today, optimized processing techniques and numerous different device concepts allow to fabricate lasers with tailored properties. The majority of applications require singlesection SLs which provide continuous wave (cw) emission or can be modulated with the injection current. Accordingly, main issues in device development are the laser linewidth, the modulation properties, or simply the output power, to name just a few.

Despite the refined fabrication methods, the dynamical behavior of SLs still remains a field of intense research. Already early investigations of different SL types have shown that their spectral properties are strongly affected by external perturbations. In particular, the problem of optical feedback has attracted much attention, since it occurs in numerous experimental situations. Examples are the reflections from an optical disc or the backscatter from optical fibers. Initiated by the seminal paper of Lang and Kobayashi in 1980 [58], the feedback induced effects for many configurations have been investigated. This research established a comprehensive understanding of nonlinear phenomena in the SL. As a consequence, SLs have become a preferred testing ground for nonlinear dynamics theory. Most of the research regarding delayed optical feedback to date aims at feedback lengths from many meters down to a few centimeters. Feedback setups with these external cavity lengths are interesting because of the very complex and irregular dynamics they can provide. A practical application is the generation of chaotic optical signals for encrypted communication [67]. The regime of very short feedback delay has attracted much less attention due to the expected trivial dynamics. Regular high-frequency pulsations have been predicted for strong feedback and very short feedback cavities [82], but were not experimentally employed so far.

The generation of optical pulses is of major interest due to the countless applications. Pulse sources are required in many fields, ranging e.g. from spectroscopy, where the laser wavelength and the pulse width are important, to optical data communica- 


\section{Introduction}

tion. Here, pulses with high repetition rates up to several tens of $\mathrm{GHz}$ and a timing stability in the sub-ps regime are needed. For communication applications typically mode-locked lasers (MLL) [3] or self-pulsating lasers (SPLs) [63] are used. These laser types employ different mechanisms for pulse generation. In the MLL several longitudinal cavity modes are coupled using a saturable absorber. This results in short pulses, however, with a fixed frequency defined by the device geometry. The SPLs distinguish from MLLs by tunable pulsations from several GHz up to several tens of GHz. Before the investigations presented in this thesis the typical SPL comprised two distributed feedback (DFB) lasers in a multi-section device. In general, the DFB sections have different gratings and thus allow for a tailoring of the laser dynamics. Two characteristic pulsation phenomena have been demonstrated. Dispersive Q-switching allows for frequencies in the range of the relaxation oscillations up to $10 \mathrm{GHz}$ [8]. Higher frequencies can be achieved by heterodyning two distinct modes generated by the DFB sections [64].

The flexibility in frequency tuning and the generation of pulses with high timing stability makes the SPL attractive as pulse source. Fields of application are optical transmitters or the use as all-optical clock recovery in optical networks. In these areas, the SPLs have become key components for future all-optical communication networks at $40 \mathrm{Gbit} / \mathrm{s}$ and higher data rates [10].

SPLs are developed and fabricated mainly at the Heinrich-Hertz-Institut für Nachrichtentechnik $(\mathrm{HHI})^{1}$, Berlin. In order to understand and optimize the SPLs, a close research cooperation with physicists at the Humboldt-University and mathematicians at the Weierstrass-Institut (WIAS) in Berlin has been established. This cooperation covers the whole range from theory to the fabrication, characterization, and the final test of the lasers in different applications. Within the last decade, the theoretical understanding and experimental know-how enabled an enormous progress. Today, a whole family of multi-section SPLs and the appropriate modeling tools to investigate and improve these devices exist.

Within this network of theoretical and experimental expertise the origins of this thesis can be found. The initial task was the improvement of the existing devices comprising two DFB lasers. My role as experimentalist was the integration of the theoretical and experimental research, translating new insights into improved devices. The simulation tools played a major role in this process, since they allowed for the easy verification of new ideas and concepts. The investigations started with a fundamental analysis of the pulsation mechanisms in multi-section lasers employing detuned DFB lasers. It turned out that a delayed feedback setup with a short feedback cavity should provide similar dynamics, while being much simpler in fabrication. Although this idea was already proposed by Tager and Petermann [82], an experimental proof was lacking. Consequently, a DFB laser with an integrated passive feedback section was realized for the experimental verification. Pulsations with frequencies in the range of $20 \mathrm{GHz}$ were obtained, which is not appropriate for the application in $40 \mathrm{Gbit} / \mathrm{s}$ data networks. The low feedback strength, which is only adjustable in a small range was identified as

\footnotetext{
${ }^{1}$ Since 2003: FhG-HHI
} 
a main limitation. To solve these problems, a novel device type has been developed which integrates an additional amplifier in the feedback section. On the course of investigating this device, it turned out that regular beating phenomena in the desired frequency range of $40 \mathrm{GHz}$ are only a small fraction of the manifold dynamics. In consequence, this thesis is dedicated to the investigation of nonlinear dynamics arising in lasers comprising a very short active feedback section.

The aim of the present work is to demonstrate that lasers integrating a monolithic feedback section in the non-zero short delay limit can provide complex dynamics. Undamped relaxation oscillations and the beating of laser modes are demonstrated as the dominant mechanisms giving rise to dynamical instabilities. It is shown that active optical feedback is essential for the preparation of the whole spectrum of dynamics for lasers with very short feedback cavities. A detailed experimental analysis of previously unexplored dynamics is carried out. In this way, the thesis completes the research on delayed feedback effects in semiconductor lasers, a field widely investigated in the last 25 years.

This thesis is organized as follows. Starting point in Chapter 2 is the coupled-mode theory to describe the basic behavior of a DFB laser. A detailed analysis of the mode structure arising from the distributed feedback is given. The experimental characterization of the employed DFB structures confirms the theoretical considerations on the laser modes. Additionally, the most important parameters of the laser structure are extracted. Based on the results for solitary DFB lasers, optical feedback is investigated in Chapter 3. Modifications of the static modes as well as the impact of feedback on the dynamics will be discussed. The focus lies on the regime of very short feedback delay. Undamped relaxation oscillations (ROs) and mode beating (MB) oscillations are shown to be the generic pulsation types. A DFB laser with an integrated passive feedback section is realized. It allows for the experimental demonstration of the characteristic pulsations in the short delay limit.

Since the device setup with passive feedback shows strong limitations regarding the control of feedback strength, Chapter 4 presents a new device: the active feedback laser (AFL). This laser type integrates an additional amplifier section in the compound-cavity; in this way, full control over the feedback parameters strength and phase is achieved. Experiments show that the AFL can provide RO and MB pulsations. Numerical simulations confirm the basic measurement results, making the further investigation of the device reasonable. An experimental setup allowing for a comprehensive characterization of the AFL is described.

To supplement the measurement results, a bifurcation diagram for the AFL is presented in Chapter 5. The most prominent bifurcation types of stable and unstable states are discussed with respect to the dynamics in the investigated laser system. Guided by the bifurcation overview, Chapter 6 reviews the experimental results. Hysteretic behavior due to coexisting laser states is investigated. Hopf bifurcations are shown to induce both $\mathrm{RO}$ and $\mathrm{MB}$ pulsations. In the torus regime these two oscillation types can coexist. Locking phenomena and chaotic behavior due to the mutual interaction of $\mathrm{MB}$ and $\mathrm{RO}$ are demonstrated in Chapter 7. Simulations enable the identification of a boundary crisis scenario in the torus break-up regime. 


\section{Introduction}

Chapter 8 gives examples where noise induced phenomena can be observed in the AFL. Noisy precursors and irregular emission at the transition from RO to MB pulsations induced by internal noise are presented. The synchronization of AFL dynamics to external optical signals is briefly sketched in Chapter 9, since it is the basis for the technical application of these devices. Finally, Chapter 10 gives a summary. 


\section{Chapter 2}

\section{Distributed feedback laser}

Distributed feedback (DFB) lasers are the essential part of the multi-section lasers investigated in this work. This Chapter summarizes the most important characteristics of DFB lasers. It is shown that wavelength selective feedback results in modes with different threshold gain and enables stable single-mode lasing. Rate equations are introduced to describe single-mode laser dynamics. In the experimental part of this Chapter the DFB laser structure is presented and the most relevant device parameters are determined. A focus lies in the investigation of longitudinal spatial hole burning (LSHB).

\subsection{Theoretical description of the DFB laser}

A complete description of semiconductor laser (SL) dynamics requires a quantummechanical treatment of both, the electromagnetic field and the gain medium. This leads to a problem of enormous complexity. Such an ab-initio investigation is dispensable for most investigations, since the large optical intensities allow for a classical description of the electromagnetic field. Maxwell-Bloch equations [89, 61] are commonly used to account for the interaction of light with the laser medium. They describe the electrical field, the material polarization and the inversion in the laser. In semiconductor materials the polarization relaxes with time scales in the order of $100 \mathrm{fs}$, being much faster than the other variables dominating the laser dynamics. Therefore, the polarization can be adiabatically eliminated if only the slower variables are of interest [41]. Thus, one ends up with two equations. One equation describes the complex electromagnetic field, which has to obey the Maxwell equations. The other is a rate equation accounting for the inversion dynamics. An appropriate theoretical framework for the investigated DFB lasers is now discussed.

\subsubsection{Traveling-wave equations}

Edge emitting SLs have a gain spectrum much broader than the spacing of longitudinal Fabry-Perot modes. A way to ensure dynamically stable single-mode emission is frequency selective feedback. It can be realized by a periodical corrugation of the 


\section{Distributed feedback laser}

waveguide, which couples forward- and backward-traveling waves in the laser resonator. This makes the resonator losses or gain strongly wavelength dependent. In practice, such a periodic modification is introduced by a grating etched into the waveguide or the active layer. Within this work only index-coupled lasers are investigated, where the waveguide is periodically structured to give an index variation with periodicity $\Lambda$ (Fig. 2.1). The coupled-mode theory is an appropriate description of contra-directional coupling in the laser cavity.

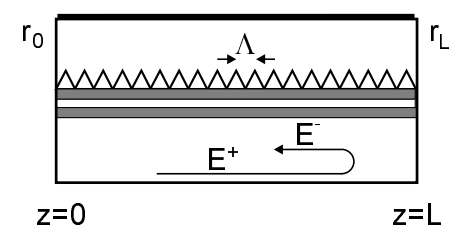

Figure 2.1: Schematic of a DFB laser. The active layer is embedded in a pair of optical waveguides. Index-coupling is realized by a periodic corrugation of the upper waveguide.

\section{The optical field}

The grating mainly influences the longitudinal propagation of the optical field. With respect to the investigated lasers, it is assumed that the mode is strongly guided and the laser oscillates in the fundamental transverse mode. Due to the stable lateral distribution of the optical field it plays no role for the device dynamics and can be separated. The longitudinal propagation of the optical wave with a frequency $\omega$ along the waveguide $(z \in[0, L])$ is characterized by a propagation constant

$$
k(\omega, n)=\frac{\omega}{c} n_{e f f}(\omega, n)+\frac{i}{2}\left(g(\omega, n)-\alpha_{0}\right) .
$$

Here, $n_{\text {eff }}$ denotes the effective refractive index, $g$ the optical gain (incorporating the transverse confinement factor $\Gamma), \alpha_{0}$ the waveguide losses, and $c$ the speed of light. Gain as well as refractive index can depend on the frequency $\omega$ and the carrier density $n$.

In the investigated DFB lasers, the waveguide is modified by a first-order Bragggrating with period $\Lambda$. It couples the forward- and backward-traveling waves in the cavity. Hence, the total optical field in the laser can be described by a superposition of two counter-propagating waves:

$$
E(z, t)=\left[E^{+}(z, t) e^{-i \pi z / \Lambda}+E^{-}(z, t) e^{+i \pi z / \Lambda}\right] e^{i \omega_{0} t} .
$$

The spatial variation due to the grating is taken into account by a propagation constant $\beta_{B}=\pi / \Lambda$. $\omega_{0}$ is the central frequency of the optical wave packet. It can be chosen arbitrarily; for convenience it will be later identified with the emission frequency of 
the solitary laser. $E^{ \pm}(z, t)$ are slowly varying amplitudes of forward- and backwardtraveling waves along the longitudinal axis of the laser. The field amplitudes have to fulfill the traveling-wave equations (TWEs) [84]

$$
+\frac{i}{v_{g}} \partial_{t} E^{ \pm}=\left(\mp i \partial_{z}+\left[k\left(\omega_{0}, n\right)-\frac{\pi}{\Lambda}\right]\right) E^{ \pm}+\kappa^{ \pm} E^{\mp}
$$

Here, $\partial_{t}$ and $\partial_{z}$ represent the partial derivatives with respect to time $t$ and longitudinal position $z, v_{g}$ is the group velocity. The strength of coupling is characterized by the coupling coefficient $\kappa^{ \pm}$. For homogeneous gratings $\kappa$ is constant within the DFB section. Noise is neglected here, because the main interest lies in the basic DFB characteristics. The Eqs. (2.3) have to be supplemented by boundary conditions at the laser facets. Considering a perfectly anti-reflection (AR) coated DFB laser with facet amplitude reflectivities $r_{0, L}=0$, the proper boundary conditions are $E^{+}(0, t)=$ $E^{-}(L, t)=0$.

\section{Coupled-mode equations}

Generally, the emission of a laser is governed by the resonator modes. In case of DFB lasers, these modes are solutions of the homogeneous TWEs and depend exponentially on time. The solutions of the TWEs can be decomposed in a spatial and a temporal part by

$$
E^{ \pm}(z, t)=\Phi^{ \pm}(z) e^{i \Omega t}
$$

Real and imaginary part of the complex mode frequency $\Omega$ describe the deviation from the reference frequency $\omega_{0}$ and the intensity decay rate, respectively. Using the decomposition Eq. (2.4) for stationary states, the TWEs reduce to the coupled-mode equations

$$
\left(\mp i \partial_{z}+\beta\right) \Phi^{ \pm}+\kappa^{ \pm} \Phi^{\mp}=0 .
$$

The propagation constant $\beta$ here accounts for the grating and the complex mode frequency by

$$
\beta=k\left(\omega_{0}, n\right)+\frac{\Omega}{v_{g}}-\frac{\pi}{\Lambda}=\beta_{0}+\frac{\Omega}{v_{g}} .
$$

\section{Resonator modes}

The mode structure of the DFB laser results from the coupled-mode equations. By setting the boundary conditions at the right facet to $E^{-}(L, t)=0$, the DFB reflectivity $r$ is obtained as ratio of out- and ingoing fields $E^{-} / E^{+}$at the left facet from Eq. (2.5) as $[52,23]$

$$
r(\beta L)=\frac{\kappa L \sin \gamma L}{i \gamma L \cos \gamma L-\beta L \sin \gamma L} .
$$

Here, $\kappa$ is the real coupling coefficient for the index grating, $L$ the device length, and $\gamma$ is defined by $\gamma^{2}=\beta^{2}-\kappa^{2}$. Thus, the reflectivity of the DFB section only depends 


\section{Distributed feedback laser}

on the device length and the coupling coefficient. The parameter $\kappa L$ is therefore an appropriate value to describe DFB lasers. Non-trivial solutions of the TWEs are obtained when the denominator in Eq. (2.7) becomes zero. This is equivalent to the zeroes of the inverse reflectivity $q$ :

$$
q(\beta L)=\frac{1}{r(\beta L)}=\frac{i \gamma L}{\kappa} \cot \gamma L-\frac{\beta L}{\kappa L}=0 .
$$

In general, this equation has to be solved numerically. As an example, the lowest threshold modes for $\kappa L=2.6$ are depicted in Fig. 2.2 by the real and imaginary part of $\beta_{m} L$. The set of solutions $\beta_{m} L$ completely determines the mode spectrum of the

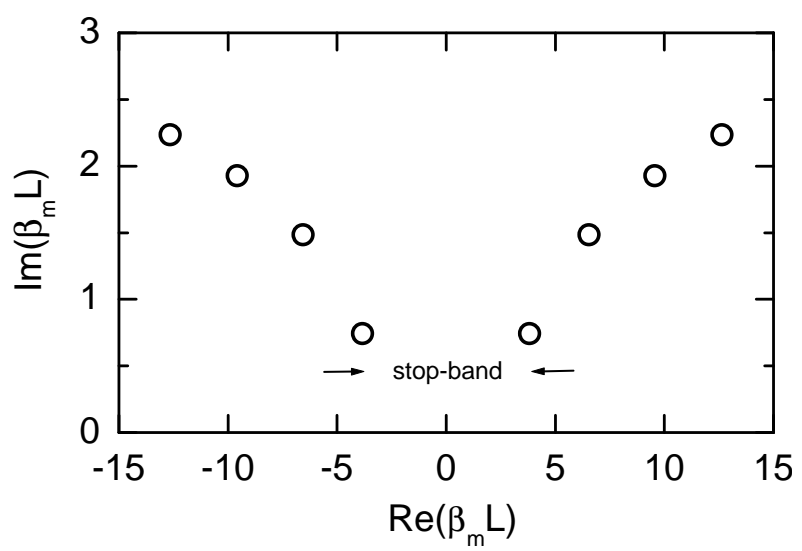

Figure 2.2: Real and imaginary part of $\beta L$ for a DFB laser $(\kappa L=2.6)$. Modes are located symmetrically to $\operatorname{Re}(\beta L)=0$. The stop-band is defined by the wavelength difference of the lowest threshold DFB modes.

DFB laser for a given value of $\kappa L$. Threshold gain and wavelength of the different modes $m$ are defined by

$$
\beta_{m} L=\left[\beta_{0}+\alpha_{H} \frac{g_{m}}{2}+\operatorname{Re} \frac{\Omega_{m}}{v_{g}}\right] L+i\left[\frac{1}{2}\left(g_{m}-\alpha_{0}\right)+\operatorname{Im} \frac{\Omega_{m}}{v_{g}}\right] L .
$$

The Henry linewidth enhancement factor $\alpha_{H}$ enters the real part of the propagation constant. It relates the changes of refractive index $n_{\text {eff }}$ and gain $g$ due to variations of the carrier density $n$ by

$$
\alpha_{H}=\frac{2 \omega}{c} \frac{\partial n_{e f f} / \partial n}{\partial g / \partial n} .
$$

For semiconductor lasers $\alpha_{H} \neq 0$ holds; typical values lie in the range of $-2 \ldots-10$. This results in a strong coupling of phase and intensity of the optical field, which is responsible for many phenomena arising in semiconductor lasers.

Since the solutions of the coupled-mode equations are of exponential type $\sim e^{i \Omega t}$, stationary lasing states require $\operatorname{Im}(\Omega)=0$. This condition defines the threshold gain 
$g_{m}$ and the lasing wavelength for the different resonator modes by

$$
\begin{aligned}
g_{m}^{t h} & =\alpha_{0}+2 \operatorname{Im} \beta_{m}, \\
\operatorname{Re} \frac{\Omega_{m}}{v_{g}} & =\operatorname{Re} \beta_{m}-\beta_{0}-\alpha_{H} \frac{g_{m}^{t h}}{2} .
\end{aligned}
$$

By the threshold gain the carrier density of the different solutions is defined. The laser typically emits on the mode $l$ showing lowest threshold and the gain is fixed at the threshold value $g=g_{l}^{t h}$. Hence, all other modes of the laser are below threshold, with a damping $\operatorname{Im}\left(\Delta \Omega / v_{g}\right)=\operatorname{Im}\left(\beta_{m}-\beta_{l}\right)>0$. The lasing process is therefore dominated by the mode having the lowest threshold gain.

Equation (2.8) defines pairs of modes with equal threshold located symmetrically around the Bragg-wavelength. Therefore, two lowest threshold modes occur in an AR coated DFB laser with homogeneous index grating. A situation with two modes having equal gain is unstable and small perturbations, e.g. due to a residual facet reflectivity, prefer one of the stop-band modes. A controlled definition of the lasing mode can be achieved by a detuning between the maximum gain- and Bragg-wavelength. Investigations in the experimental part of this Chapter will show that an inhomogeneous carrier density distribution due to spatial hole burning in general stabilizes the short wavelength stop-band mode.

Figure 2.3 shows the dependence of real and imaginary part of $\beta_{t h} L$ for the modes with lowest threshold. Both, threshold gain and the lasing wavelength of the modes depend on the value of $\kappa L$. The offset from the Bragg-wavelength increases with $\kappa L$,

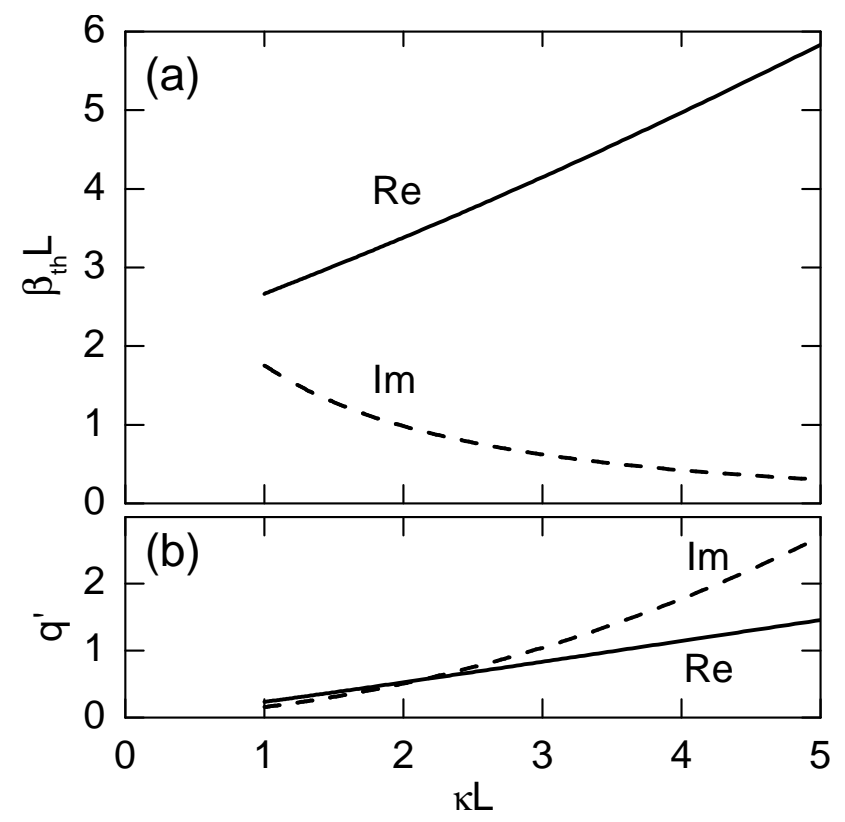

Figure 2.3: Characteristics of a DFB laser. (a) Real and imaginary part of $\beta_{t h} L$; (b) first derivatives $q^{\prime}$ of the inverse reflectivity at threshold with respect to $\beta L$.

whereas the lasing threshold decreases. The decrease in $g_{t h}$ corresponds to lower cavity 
losses; the threshold gain determines the photon lifetime $\tau_{p}$ by $g_{t h}=1 / \tau_{p}$.

External influences modify the stationary laser state. Restricting to small perturbations allows the use of linear approximations. For later investigations the values of the inverse reflectivity slope $q^{\prime}$ with respect to $\beta L$ at the position of the lowest loss mode are indicated in Fig. 2.3.

\subsubsection{Dynamics of optical modes}

The analysis of the coupled-mode equations has shown that frequency selective feedback in the DFB laser can provide single-mode emission. Consequently, all following considerations will focus on a single lasing mode. For convenience, the central frequency $\omega_{0}$ and the carrier density $n$ are fixed to the values at threshold by requiring

$$
k_{0}\left(\omega_{0}, n_{t h}\right)=\beta_{l},
$$

where $\beta_{l}$ denotes the lasing mode and $n_{t h}$ the threshold carrier density. Small deviations from the threshold values can be described by a linear approximation of the propagation constant

$$
\beta(\Omega, N)=\beta_{l}+\frac{\Omega}{v_{g}}+\frac{1}{2}\left(i+\alpha_{H}\right) g^{\prime} N .
$$

$N$ and $\Omega$ indicate the deviation from the threshold carrier density and lasing wavelength, respectively. By using the differential gain $g^{\prime}$, a linear relation between carrier density and gain is assumed. This is a good approximation for bulk lasers [25]. The optical field instantaneously follows any perturbation, e.g. in the carrier density, due to the different time scales defined by the inversion dynamics and the photon life-time. Hence, the condition $\beta(\Omega, N)=\beta_{l}$ is always fulfilled. This allows to describe the time dependency of the electrical field amplitude [66] by

$$
\frac{d}{d t} E(t)=i \Omega(N) E(t)=\frac{1}{2}\left(1-i \alpha_{H}\right) v_{g} g^{\prime} N E(t) .
$$

Due to the interaction with the optical field, also the carrier density becomes time dependent. For the deviation from the threshold value one obtains:

$$
\frac{d}{d t} N(t)=\frac{I-I_{t h}}{e V}-\frac{N}{\tau_{d}}-\left[\frac{1}{\tau_{p}}+v_{g} g^{\prime} N\right]|E(t)|^{2}
$$

$I$ and $I_{t h}$ denote injection and threshold current, respectively; additional parameters are the volume $V$ of the active layer, the electron charge $e$, and the photon-lifetime $\tau_{p}$. The differential carrier lifetime $\tau_{d}$ is defined by $1 / \tau_{d}=A+2 B n+3 C n^{2}$ and accounts for all channels of spontaneous recombination (A: non-radiative, B: bi-molecular, $\mathrm{C}$ : Auger recombination). $E(t)$ is normalized in such a way that the intensity $S=|E|^{2}$ equals the photon density in the laser cavity. The $\kappa L$-dependent threshold gain of a DFB laser enters the rate equations with the photon life-time $\tau_{p}$.

A few simple relations describing the laser behavior can be deduced from the rate equations. The trivial solution becomes unstable at the laser threshold $I_{t h}=e V n_{t h} / \tau_{d}$, 
resulting in a steady state cw emission with

$$
S=\left(I-I_{t h}\right) \tau_{p} / e V
$$

The carrier density remains clamped at the threshold value. The linear increase of the output power is in good agreement to experimental results.

A stability analysis of the rate equations shows that perturbations from a stationary state decay exponentially with the relaxation oscillation $(\mathrm{RO})$ angular frequency $\omega_{R O}$ and the damping rate $\gamma_{R O}[32,69]$ :

$$
\begin{aligned}
\omega_{R O} & =\sqrt{v_{g} g^{\prime} S / \tau_{p}}, \\
\gamma_{R O} & =\frac{1}{2}\left(\frac{1}{\tau_{d}}+v_{g} g^{\prime} S\right) .
\end{aligned}
$$

In a solitary semiconductor laser the relaxation oscillation frequency $f_{R O}=\omega_{R O} /(2 \pi)$ is in the order of several $\mathrm{GHz}$, with a damping rate $\gamma_{R O}>0$ under all injection conditions. Self-sustained oscillations therefore do not occur.

More complex dynamics (neglecting the spatial dynamics by LSHB [97, 79]) in semiconductor lasers requires additional degrees of freedom. Examples are the modulation of the injection current [59], the injection of light [92], adding a saturable absorber [31], or external optical feedback [58].

\subsubsection{Simulation tool LDSL}

So far, the theoretical framework illustrated the mode selection mechanisms in the DFB laser and the basic dynamical behavior. Several simplifications were assumed, including homogeneous carrier densities. Aiming at a quantitative modeling of DFB and multi-section lasers, an appropriate description has to rely directly on the TWEs and the corresponding carrier density equation. Additional effects like spontaneous emission noise, inhomogeneous carrier density distribution, gain dispersion and nonlinear gain saturation have to be taken into account. Appendix A.3 gives details on the implementation of these effects.

The software suite LDSL (Longitudinal Dynamics in multisection Semiconductor Lasers) of the Weierstrass Institute ${ }^{1}$ implements a comprehensive TWE model for multi-section lasers. LDSL allows the simulation of laser dynamics with high accuracy, enabling qualitative and quantitative predictions of dynamical phenomena. Throughout this work it is used for the simulation of the investigated structures. Additionally, LDSL is employed to supplement experimental results where a direct measurement of laser parameters is not possible. Here, simulations help to clarify the underlying physics.

\footnotetext{
${ }^{1}$ http://www.wias-berlin.de/software/ldsl/
} 


\subsection{Experimental characterization}

\subsubsection{Design of investigated lasers}

Switching of the laser modes due to small perturbations as it appears in FabryPerot (FP) lasers complicates the investigation of feedback effects. As a consequence, throughout this work DFB lasers and adequate multi-section modifications thereof are used. Figure 2.4 shows a schematic of the employed DFB structure.

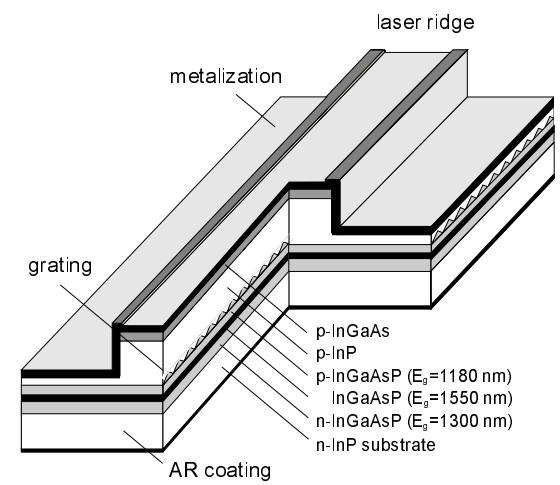

Figure 2.4: Schematic illustration of ridge-waveguide DFB laser. Multi-section devices are modifications of this design.

The lasers are based on a InGaAsP-InP bulk hetero-structure grown by low-pressure metal-organic vapor phase epitaxy. The peak gain wavelength of the $150 \mathrm{~nm}$ thick InGaAsP active layer is centered at $1550 \mathrm{~nm}$, owing to the absorption minimum of optical fibers. A bulk active layer is sandwiched in an asymmetric 1180-nm-/1300-nmInGaAsP optical waveguide of $150 \mathrm{~nm}$ and $250 \mathrm{~nm}$ thickness, respectively. Lateral and transversal single-mode lasing is achieved by a ridge waveguide of $2.4 \mu \mathrm{m}$ width and $1.5 \mu \mathrm{m}$ height. Frequency selective feedback is introduced by structuring the upper waveguide by e-beam lithography and reactive ion etching. Subsequently, the homogeneous first-order index grating is overgrown with InP. The coupling coefficient $\kappa$ is defined by the etching depth of the grating, which is typically $100 \mathrm{~nm}$. Low- and high-index regions of the grating have equal lengths. TE polarization is enhanced by a slight compressive strain of the active layer to suppress TE/TM lasing competition (TE: transversal electric, i.e. the electrical field lies in the plane of the active layer, accordingly TM: transversal magnetic). Both facets are AR coated to eliminate additional lasing of Fabry-Perot modes. Typical values of the power reflectivity $R$ for AR coated facets are $10^{-4}$. The lasers are thinned to $125 \mu \mathrm{m}$ and soldered to a diamond heatsink to enable a fast heat-transfer away from the active layer. The untapered waveguides at the device facets require lensed single-mode optical fibers to increase the coupling efficiency. A characteristic value for the losses at the laser/fiber interface is $6 \mathrm{~dB}$. These coupling losses have to be taken into account when modeling and measurement results are compared. 


\subsubsection{Parameters of investigated laser structure}

This Section summarizes the key parameters which are later required for the design of multi-section lasers. Additionally, these values serve for realistic simulations. All subsequently investigated test-structures, DFB lasers and multi-section devices are realized from a single wafer, guaranteeing consistent parameters. For the following investigations the laser structures are clamped to a heatsink and the temperature is controlled to $20^{\circ} \mathrm{C}$. The laser output is coupled to an optical fiber. Optical spectra are characterized using an Advantest Q8384A optical spectrum analyzer (OSA). A calibrated HP71400C Lightwave Analyzer is used to measure the dynamical characteristics of the DFB lasers.

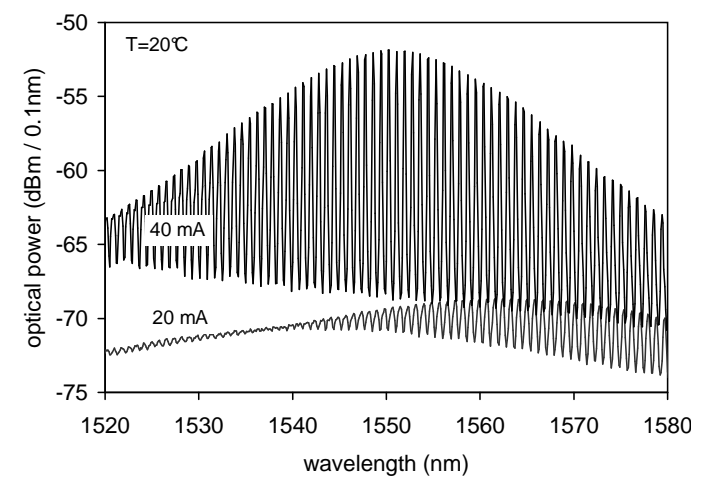

Figure 2.5: Optical spectra for a FabryPerot laser (facet reflectivity $R=0.3$ ).

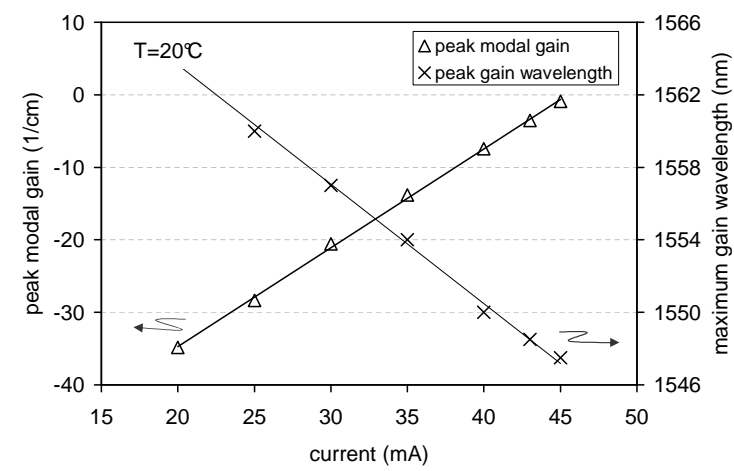

Figure 2.6: Current dependence of peak modal gain and wavelength.

Optical gain and losses are the fundamental properties of a semiconductor laser. Fabry-Perot lasers are used for the characterization. ${ }^{2}$ The wavelength dependence of the amplified spontaneous emission (ASE) intensity of a FP laser is given by

$$
I(\lambda) \propto \frac{\left(1+\operatorname{Re}^{\left(g-\alpha_{0}\right) L}\right)(1-R)}{\left(1+R e^{\left(g-\alpha_{0}\right) L}\right)^{2}-4 \operatorname{Re}^{\left(g-\alpha_{0}\right) L} \sin ^{2}\left(\frac{2 \pi n_{g r} L}{\lambda}\right)} .
$$

It depends on the laser length $L$, the gain $g$, the facet reflectivity $R$, the internal losses $\alpha_{0}$, and the group refractive index $n_{g r}$. The proportionality is defined by the amount of spontaneous emission coupled into the lasing modes. From Eq. (2.20) the modal gain $G(\lambda)=g(\lambda)-\alpha_{0}-\alpha_{\text {mirror }}$ can be determined as [42]

$$
G(\lambda)=\frac{1}{L} \ln \frac{\sqrt{r(\lambda)}-1}{\sqrt{r(\lambda)}+1}
$$

where $r(\lambda)=\frac{I_{\max }(\lambda)}{I_{\min }(\lambda)}$ denotes the peak-to-valley ratio of adjacent ASE ripples. Figure 2.5 shows TE-ASE spectra measured for a $400 \mu \mathrm{m}$ FP laser and different injection

\footnotetext{
${ }^{2}$ The FP lasers used here are identical to the DFBs, however, omitting the grating. Cleaved facets act as mirrors.
} 
currents below threshold. The contrast of the FP resonances scales with the gain of the active section. Band-filling shifts the peak position to shorter wavelengths. The peak modal gain increases linearly with the injection current, until threshold $(G=0)$ is reached at $47 \mathrm{~mA}$ (Fig. 2.6). A full-width at half-maximum (FWHM) of $30 \mathrm{~nm}$ for the gain spectrum at threshold results from the low gain dispersion of the bulk active layer.

Since the mirror losses of an as-cleaved FP laser only depend on the facet reflectivity $R$, the material gain can be determined once the internal losses $\alpha_{0}$ are known. A method proposed by Andrekson [2] allows the determination of losses from measurements on a single device by using its detection properties. At a bias below the point of material transparency, i.e. $g=0$, photons of a modulated probe light are absorbed (provided a wavelength within the gain spectrum); above transparency stimulated emission occurs. Hence, the transparency current can be detected by a phase change of the diode junction voltage of $180^{\circ}$. The ASE ripple at transparency determines the material gain, which is equal to the losses. An average value of $25 \mathrm{~cm}^{-1}$ has been obtained for wavelengths in the range from $1530 \mathrm{~nm}$ to $1550 \mathrm{~nm}$. Taking into account the mirror losses for the FP device with $\alpha_{\text {mirror }}=(1 / L) \ln (1 / R) \approx 30 \mathrm{~cm}^{-1}$, the material gain at threshold amounts to $g=55 \mathrm{~cm}^{-1}$. The values for internal loss and threshold gain are in good agreement to results from the usually applied cut-back method [16] for identical laser structures [17].

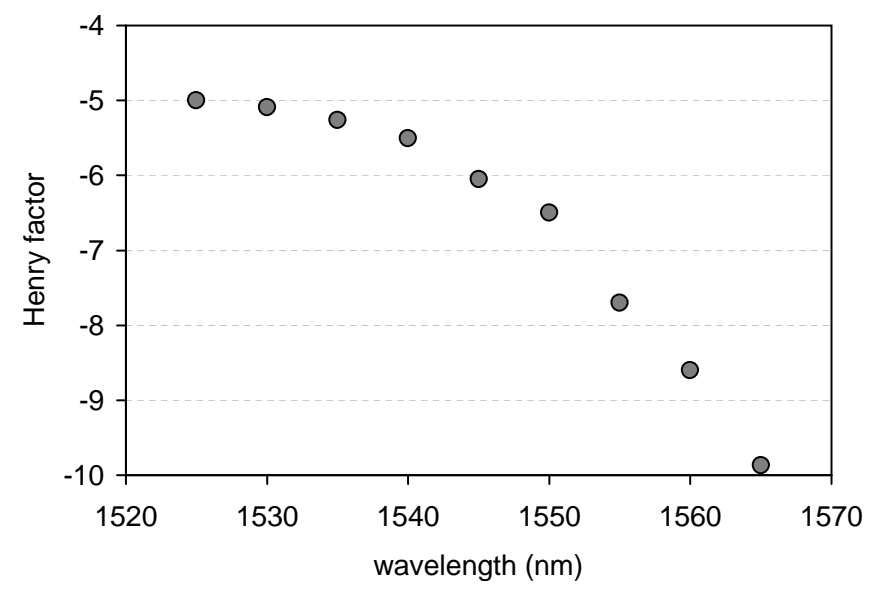

Figure 2.7: Dependence of the Henry factor $\alpha_{H}$ on lasing wavelength.

An important parameter for the dynamical behavior is the Henry factor $\alpha_{H}$. It relates the change of real and imaginary part of the refractive index due to a change in carrier density [68]. Although the carrier density within a laser is a value which is not directly accessible, $\alpha_{H}$ can be extracted from sub-threshold FP spectra. At a given wavelength, the shift $d \lambda_{i}$ and the increase in ASE modulation $d r_{i}$ of the $i$-th ASE ripple has to be determined for different injection currents [47]. Since varied injection currents correspond to well-defined carrier densities, the Henry factor results from the 
change of wavelength and modulation depth as

$$
\alpha_{H}=\frac{2 \pi}{\delta \lambda_{i}} \frac{d \lambda_{i}}{d[\ln ((\sqrt{r}-1) /(\sqrt{r}+1))]} .
$$

The spacing of FP-ripples at the $i$-th mode is denoted by $\delta \lambda_{i}$. Figure 2.7 summarizes the measurement results for the investigated laser structure. Compared to quantumwell active layers the bulk structures show large values of $\alpha_{H}$. This is a result of the low differential gain $d g / d n$ [23]. In the wavelength range of interest $(1535-1550 \mathrm{~nm})$ values of $\alpha_{H}=-5$ to -6 are found. The absolute value strongly increases towards the band-gap of the active material.

In summary, values for gain and internal losses have been determined. For the investigated structures a peak gain wavelength of $1550 \mathrm{~nm}$ at threshold and internal optical losses of $25 \mathrm{~cm}^{-1}$ have been obtained in the wavelength range employed for the DFB structures around $1540 \mathrm{~nm}$. Here, the Henry factor has a value of $\alpha_{H}=-5.5$.

\subsubsection{Single-mode operation due to LSHB}

DFB lasers can be realized with the same hetero-structure as Fabry-Perots by modifying the waveguide with an index grating. In this work, DFB lasers with homogeneous gratings and coupling coefficients $\kappa$ larger than $100 \mathrm{~cm}^{-1}$ are investigated. Already short laser cavities result in high $\kappa L$-values and require a careful adjustment of laser lengths to maintain longitudinal single-mode operation. Appropriate parameter combinations are discussed in this Section.

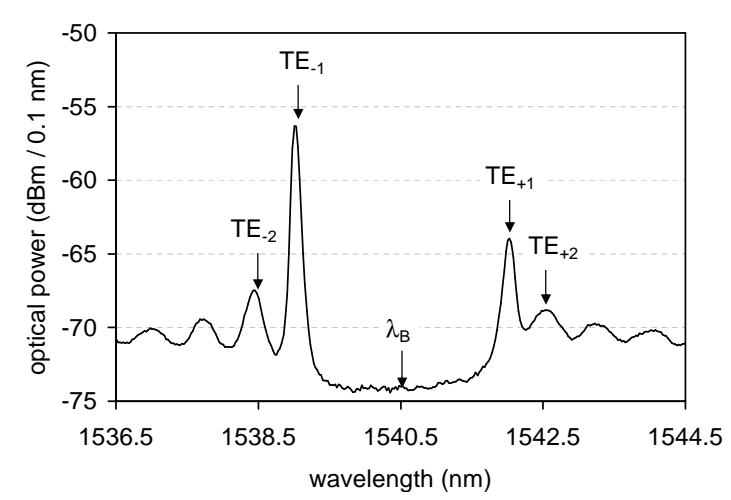

Figure 2.8: Sub-threshold optical spectrum of a $400 \mu \mathrm{m}$ DFB laser. The main spectral features are indicated for further discussion.

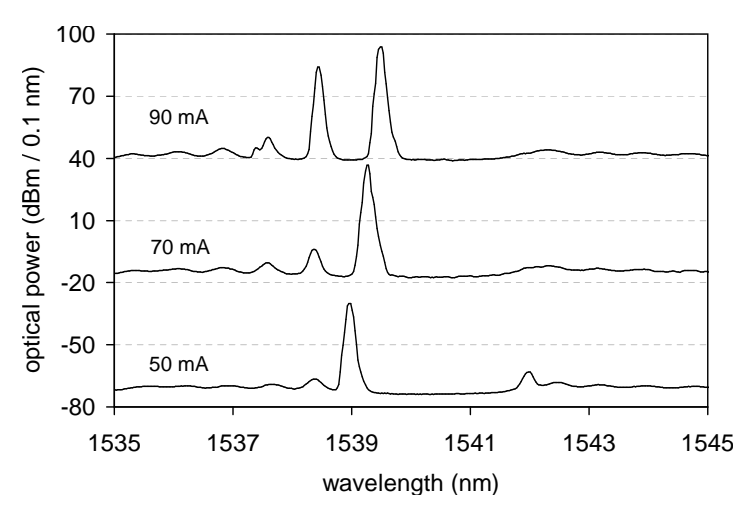

Figure 2.9: Impact of LSHB on the optical spectra ( $55 \mathrm{~dB}$ offset between spectra).

Figure 2.8 shows the TE spectrum of a $400 \mu \mathrm{m}$ DFB laser below threshold $\left(I_{t h}=\right.$ $35 \mathrm{~mA}$ ). Facets are AR coated (residual power reflectivity $10^{-4}$ ). The stop-band width is defined by the two modes $\mathrm{TE}_{-1}$ and $\mathrm{TE}_{+1}$ located symmetrically around the Braggwavelength $\lambda_{B}$. Fitting the spectrum in Fig. 2.8 with a method proposed by Wenzel in [90], a value of $\kappa=130 \mathrm{~cm}^{-1}$ is obtained, corresponding to $\kappa L=5.2$. Above-threshold 
spectra are shown in Fig. 2.9 at different injection currents. The degeneracy of stopband modes is lifted for currents already slightly above threshold. Towards higher injection, additional longitudinal modes on the short stop-band side are successively undamped. Both observations can be explained by LSHB, which has not been taken into account in the idealized considerations of Section 2.1.

The low mirror losses due to the high $\kappa L$-value cause an increased photon density in the center of the resonator. The consequence is a decrease of the local carrier density and a longitudinal variation of the refractive index (the effect is therefore called longitudinal spatial hole burning). At the center of the device, the Bragg-resonance is shifted towards shorter wavelengths with respect to the facet regions. In effect, modes at the short wavelength stop-band side have a lower threshold. The reason is, that long wavelength modes located in the laser center can not propagate in the stop-band of the outer grating regions. Equivalently, only the short wavelength modes located in the facet regions are supported by the high reflectivity of the central region. In this way, the emission of a laser showing LSHB is centered at the short stop-band side. Strong injection therefore has to be avoided for high $\kappa L$-values [74], when single-mode behavior is required.

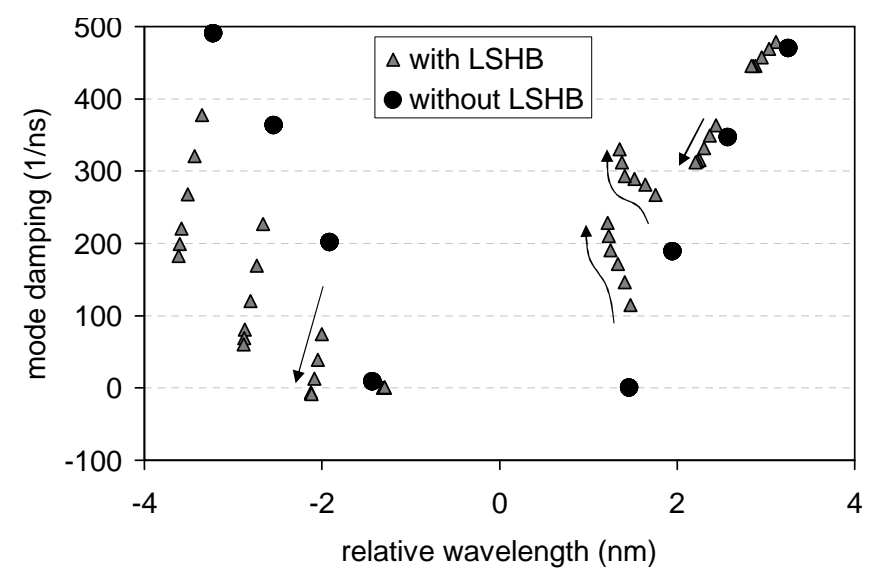

Figure 2.10: Damping of longitudinal modes with homogeneous carrier distribution and with LSHB. Arrows indicate the change for increasing current. LSHB stabilizes the emission on the short wavelength stop-band mode.

A numerical investigation of stationary LSHB effects with LDSL is very instructive. Laser parameters for simulation are chosen according to the experimental values (summarized in Appendix A.1). Figure 2.10 shows a comparison of the mode damping for currents from $50 \mathrm{~mA}$ to $100 \mathrm{~mA}$. Two situations were investigated. The idealized case of zero series resistance $R_{s}=0 \Omega$ of the cladding layers provides a homogeneous carrier distribution, although the photon density varies along the laser cavity. The mode spectrum remains symmetric with respect to the Bragg-wavelength, short and long wavelength modes have equal damping. Therefore, the simulation results are in full agreement to the coupled-mode theory. 
The second investigation takes into account a resistance of $R_{s}=5 \Omega .{ }^{3}$ Here, LSHB strongly modifies the mode spectrum. As already observed in experiment, the short wavelength mode $\mathrm{TE}_{-1}$ always shows a lower damping than $\mathrm{TE}_{+1}$, even for injection levels only slightly above threshold. Increasing injection, further modes on the left stop-band side are successively undamped, finally causing the simultaneous emission on several longitudinal modes. The different longitudinal distribution of modes results in a varying average refractive index and a shift of relative mode positions. Experimental results in Fig. 2.9 confirm the shift of modes. In experiment, the mode positions are additionally modified by the thermal change of refractive index due to current heating of the laser. An average value for the thermal wavelength shift is $0.02 \mathrm{~nm} / \mathrm{mA}$. It strongly depends on the mounting of the laser on the heatsink.

A commonly applied technique to achieve single-mode lasing is the introduction of a $\lambda / 4$ phase shift in the center of the grating. In this way, the degeneracy of the stop-band modes $\mathrm{TE}_{-1,+1}$ disappears and a single mode at the center of the stop-band exhibits the lowest threshold even without LSHB. The effectiveness of a phase shift in the DFB grating, however, is strongly dependent on a longitudinally symmetric distribution of field and carriers [26]. Since optical feedback breaks this symmetry, phase shifted DFB lasers are not appropriate candidates for such experiments. It will be shown that multi-section devices with homogeneous grating can exhibit stable lasing on the LSHB mode, provided a proper design of the feedback cavity.

\subsubsection{Solitary DFB laser dynamics}

To this point, only the static characteristics of DFB lasers has been discussed. For the later assessment of the feedback induced effects, first the solitary DFB laser dynamics has to be known.

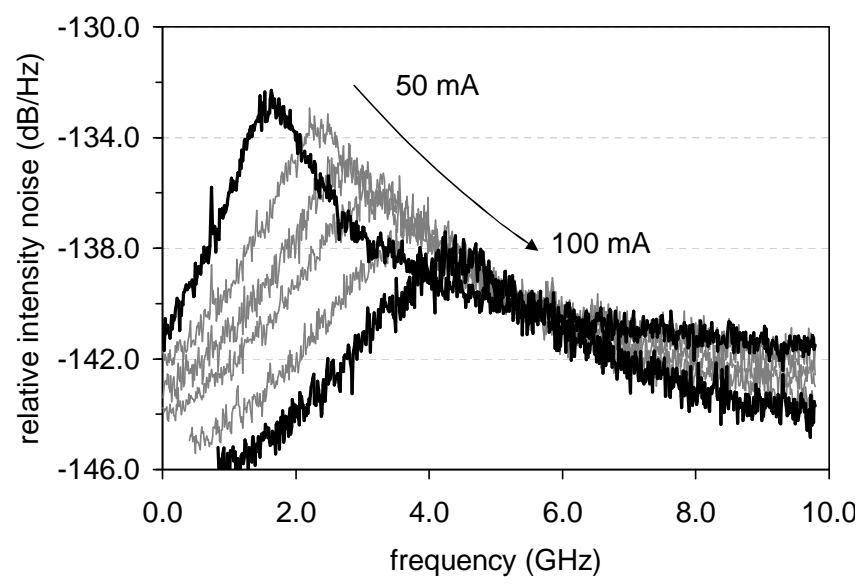

Figure 2.11: RIN spectra of a single-mode DFB laser measured for different injection currents.

\footnotetext{
${ }^{3}$ This is a typical value for the investigated devices.
} 
Relative intensity noise (RIN) measurements were carried out to characterize the dynamics of a single-mode DFB laser. The RIN is associated with the fluctuations of carrier- and photon-density caused by spontaneous emission. Deviations from the equilibrium values lead to damped oscillations with the relaxation oscillation frequency $f_{R O}$ and the damping rate $\gamma_{R O}$. Figure 2.11 shows RIN spectra for a $300 \mu \mathrm{m}$ DFB device, which is emitting single-mode $\left(\mathrm{TE}_{-1}\right)$ in the whole current range from $50 \ldots 100 \mathrm{~mA}$. The relaxation oscillation frequency and its damping determined from the RIN [38] spectra show an increase of $f_{R O}$ proportional to $\sqrt{I-I_{t h}}$ and a linear increase of the damping $\gamma_{R O}$. This is in good agreement with the predictions from the rate equations. The strongly damped relaxation oscillation dynamics is limited to frequencies up to a few $\mathrm{GHz}$ by the time constants in the laser. Self-sustained oscillations are not observed.

Besides the RIN dynamics undamped pulsations due to LSHB can occur [60]. Characteristic frequencies are in the range of $1 \mathrm{GHz}$. Pulsations due to spatial hole burning can be prevented by using moderate $\kappa L$-values and by limiting the injection current [97]. 


\section{Chapter 3}

\section{Delayed optical feedback}

The basic characteristics of single-mode DFB lasers have been introduced. This Chapter investigates the modification of solitary laser behavior under the impact of external feedback. Changes of the stationary mode spectrum and the laser dynamics are discussed. The focus lies on the phenomena occurring for very short feedback cavities. A passive feedback laser (PFL) is realized for experimental investigations. Undamped relaxation oscillations $(\mathrm{RO})$ and mode beating $(\mathrm{MB})$ oscillations are demonstrated as characteristic phenomena.

\subsection{Theoretical predictions}

It has been discussed in the previous Chapter, that small perturbations modify the modes of a DFB laser. The scenario investigated here is a DFB laser subject to external feedback, i.e. a fraction of the emitted light is re-injected into the laser cavity. Figure 3.1 sketches such a setup. Three parameters are characterizing the feedback.

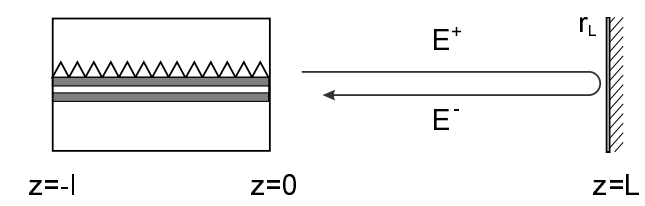

Figure 3.1: Scheme of a feedback setup. A DFB laser of length $l$ is coupled to a feedback section of length $L$.

First, the feedback strength $K$ gives the ratio of in- and outgoing field amplitudes $\left|E^{-} / E^{+}\right|$at the laser facet $(z=0){ }^{1}$ In the idealized case of no losses and a perfect coupling between feedback cavity and DFB section, the relation $K=r_{L}$ holds. The second parameter is the feedback delay time $\tau=2 L / c$, which depends on the feedback cavity length. Together with the lasing frequency $\omega_{0}$ of the solitary laser it gives the third parameter, the feedback phase $\phi=\omega_{0} \tau$.

\footnotetext{
${ }^{1}$ Please note the change of coordinates compared to the solitary DFB laser.
} 


\section{Delayed optical feedback}

Different approaches can be used to investigate the delayed feedback setup. One possibility are the full traveling-wave equations (TWEs) introduced in Section 2.1. They can be easily modified to describe external cavity setups. The boundary conditions at the feedback cavity interface $(z=0)$ are given by

$$
E^{-}(t)=K e^{-i \phi} E^{+}(t-\tau),
$$

i.e. a fraction $K$ of the field is re-injected into the laser cavity with a phase shift $\phi$ and a delay $\tau$. The resulting TWEs can be solved numerically, e.g. with the LDSL simulation tool. This allows to regard the impact of LSHB as well as effects like gain-compression and gain-dispersion. Although giving a detailed description of the experimental situation [54, 12], this approach has one important drawback: the TWEs are not very instructive and basic mechanisms are hidden by the complexity of the model.

For the understanding, it is helpful to have simple equations which qualitatively describe the dynamical phenomena. The rate equations are commonly used for such investigations. In a phenomenological approach, the equation for the optical field can be complemented by an additional feedback term accounting for the re-injected light. This results in the mean-field rate equation

$$
\frac{d}{d t} E(t)=\frac{1}{2}\left(1-i \alpha_{H}\right) v_{g} g^{\prime} N E(t)+\eta e^{-i \phi} E(t-\tau) .
$$

Here, $\eta$ denotes the feedback rate. This equation was originally introduced by Lang and Kobayashi (LK) [58] for the description of feedback effects in semiconductor lasers. By the feedback term the laser equations are transformed into a pair of coupled delaydifferential equations, which in general have complex solutions [55]. The LK equations are widely used to describe lasers with feedback. A large number of investigations have shown that this approach gives a good description in the long- and short-cavity regimes $[65,46,94]$.

The equivalence of the TWEs with modified boundary conditions and the LK rate equations can be shown [95] under the assumption of single-mode lasing and weak feedback. Hence, both approaches describe the same dynamics and can be used complementary as long as these conditions hold. In this way, investigations based on the rate equations can supplement the results obtained with the TWE model.

\subsubsection{Modes of DFB laser with feedback}

Before concentrating on the dynamical effects in feedback lasers a few considerations on the stationary states are presented. Stationary states have to fulfill the boundary condition Eq. (3.1). When restricting to single-mode lasing by requiring solutions $E^{ \pm}(t)=E_{0}^{ \pm}(\omega) e^{i \omega t}$, additionally the relation

$$
E_{0}^{+}(\omega)=r(\omega) E_{0}^{-}(\omega)
$$

holds. The optical frequency is defined by $\omega=\omega_{0}+\Omega$, where $\Omega$ describes the deviation from the solitary wavelength $\omega_{0} ; r(\omega)$ accounts for the amplitude reflectivity of the 
DFB section. The relations of forward- and backward-traveling waves result in the roundtrip condition for the compound-cavity modes:

$$
\begin{aligned}
1 & =K r e^{-i \Omega \tau} e^{-i \phi}, \quad \text { or } \\
q e^{i \Omega \tau} & =K e^{-i \phi} .
\end{aligned}
$$

The inverse amplitude reflectivity of the DFB laser is denoted by $q$. This characteristic equation allows to determine the lasing modes of the feedback setup. For moderate feedback strengths only small changes of the laser state are expected and the modes in the feedback setup are located close to the solitary positions. Accordingly, the changes of the inverse reflectivity can be approximated by

$$
\Delta q(\Omega, N)=q^{\prime} \frac{\tau_{l}}{2}\left[\Omega+\frac{1}{2}\left(i+\alpha_{H}\right) v_{g} g^{\prime} N\right]
$$

In this equation $\tau_{l}=2 l / v_{g}$ gives the roundtrip time in the DFB cavity, with $v_{g}$ as group velocity. The roundtrip condition Eq. (3.4) thus results in

$$
\left[\Omega+\frac{1}{2}\left(i+\alpha_{H}\right) v_{g} g^{\prime} N\right] e^{i \Omega \tau}=\eta, \quad \text { with } \quad \eta=\frac{2 K e^{-i \phi}}{q^{\prime} \tau_{l}} .
$$

The slope of the inverse reflectivity $q^{\prime}$ takes into account the characteristics of the DFB laser close to the lasing mode. The mode equation is a good approximation in the feedback range where Eq. (3.5) remains valid. It is obvious that Eq. (3.6) and the rate equations Eq. (3.2) describe the same behavior. A comparison of the coefficients shows that the relation $\eta=\frac{2 K}{q^{\prime} \tau_{l}}$ provides equal solutions.

The deviation of the lasing frequency $\Omega$ and the threshold gain $\Delta G=v_{g} g^{\prime} N$ from the solitary values have to fulfill the following conditions:

$$
\begin{array}{rlr}
\Omega & =\eta \sqrt{1+\alpha_{H}^{2}} \sin \left[(\Omega \tau+\phi)+\arctan \alpha_{H}\right], \quad \text { and } \\
\Delta G & =-2 \eta \cos (\Omega \tau+\phi) .
\end{array}
$$

In the phase space of lasing wavelength and carrier density, the modes therefore lie on an ellipse located around the solitary lasing mode. Changing the feedback phase $\phi$, the compound-cavity modes move on the ellipse. Increasing the feedback strength blows up the ellipse and additional modes appear. The number of solutions of the Eqs. (3.7) is determined by the feedback delay $\tau$, the feedback strength $\eta$, and the value of the Henry factor $\alpha_{H}$. A characteristic feedback parameter $C_{f}=\eta \tau \sqrt{1+\alpha_{H}^{2}}$ $[70]$ is therefore used for the further considerations.

\subsubsection{Folding of modes}

The previous investigations have shown that small feedback modifies the lasing wavelength and the threshold gain compared to the solitary values. Compound-cavity modes lie on ellipses located around the solitary mode and their number scales with $C_{f}$. Two qualitatively different situations arise, depending on the value of the characteristic feedback parameter. For values $C_{f}<1$, only one solution of the mode equations exists. 


\section{Delayed optical feedback}

This is valid for any feedback phase $\phi$ and leads to a continuous variation of the lasing wavelength. When $C_{f}$ exceeds unity, additional solutions are created as pairs of modes and antimodes. These additional modes differ with respect to their stability: modes are stable when feedback phase changes, whereas antimodes are unstable [62].
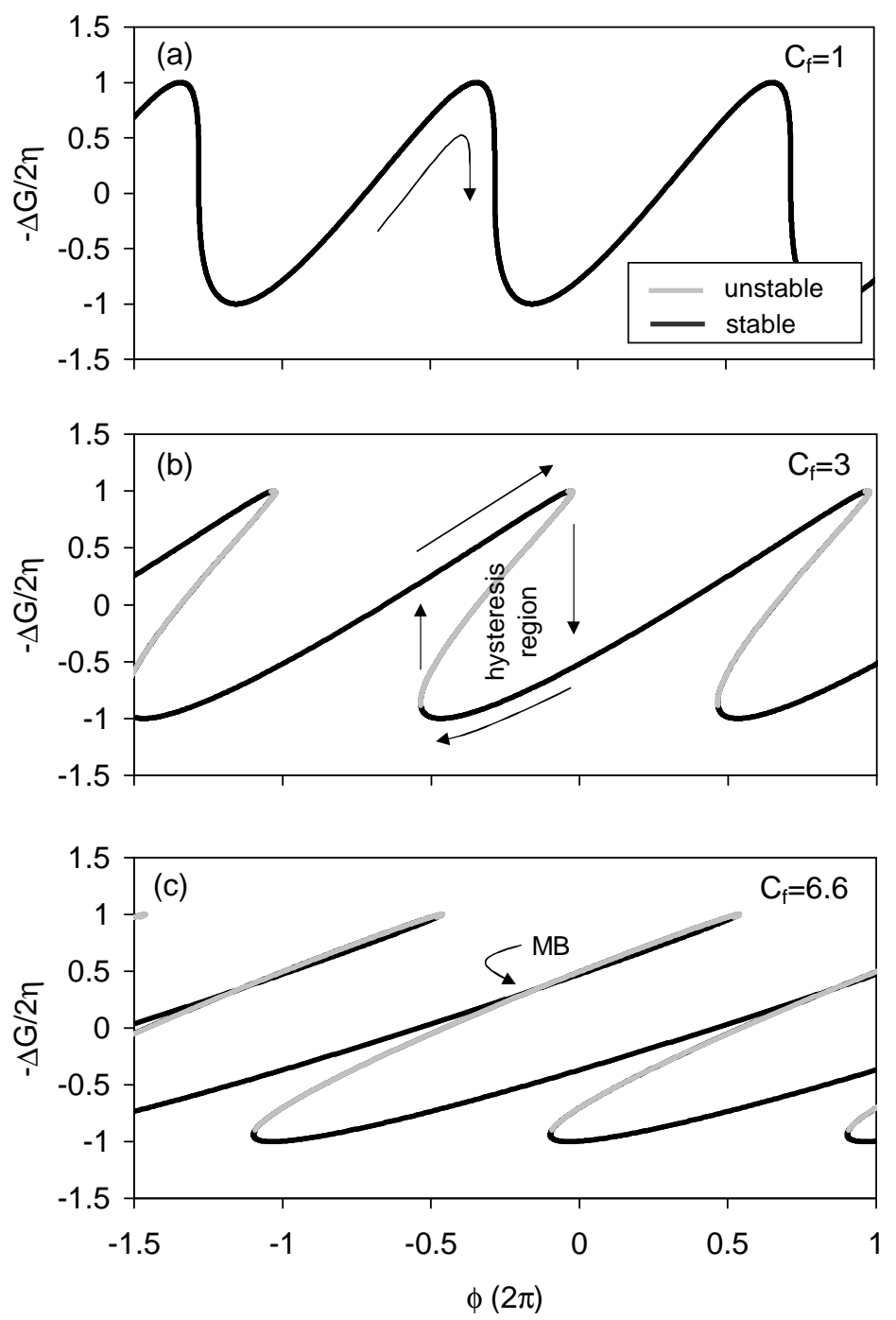

Figure 3.2: Qualitative illustration of normalized threshold gain variation vs feedback phase $\phi$. Different feedback strengths $C_{f}$ are illustrated. (a) Critical case of $C_{f}=1$, one distinct solution exists for any $\phi$; (b) $C_{f}=3$ : regions of coexisting modes give rise to hysteresis; (c) $C_{f}=6.6$ : mode beating can occur for the situation of two modes with equal threshold gain.

To illustrate the mode behavior, Fig. 3.2 qualitatively shows the normalized threshold gain variation when changing the feedback phase, i.e. the projection of the ellipse onto the threshold gain axis. The shift of lasing wavelength is omitted in this representation. Below the critical value $C_{f}=1$ one distinct solution exists and the threshold gain varies smoothly with $\phi$. Exceeding unity, the effective phase variation due to 
the change of the lasing wavelength can be larger than $2 \pi$. This results in additional modes fulfilling the roundtrip condition in the EC. In general, these modes show different threshold gain (Fig. 3.2(b)). Only for distinct feedback phase conditions a pair of mode and antimode have equal threshold gain (Fig. 3.2(c)).

The complexity of the experimental situation is strongly dependent on the number of coexisting modes. Redefining the feedback phase as $\phi_{0}=\omega_{0} \tau+\arctan \alpha_{H}$, borders

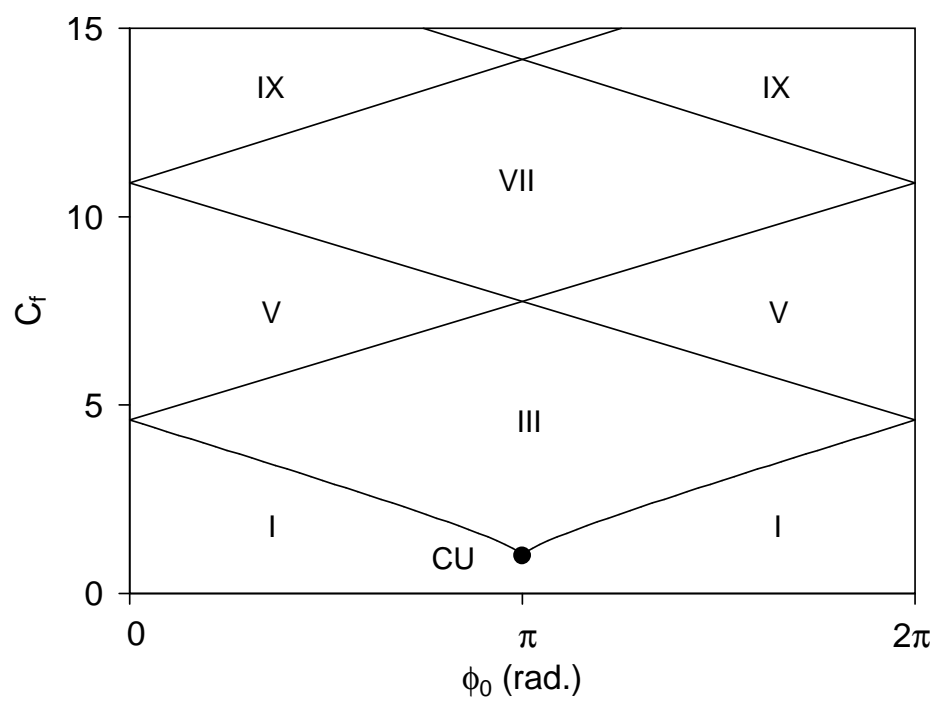

Figure 3.3: $\quad$ Borders between states with a different number of modes in the plane of feedback parameter $C_{f}$ and feedback phase $\phi_{0}$. The number of coexisting modes is indicated. $\mathrm{CU}$ defines the lower boundary for the coexistence of three modes.

between states with a different number of modes can be obtained from Eqs. (3.7) $[87,94]$ as

$$
\phi_{0}=\left[ \pm \arccos \left(\frac{1}{C_{f}}\right) \mp C_{f} \sin \left(\arccos \left(\frac{1}{C_{f}}\right)\right)\right] \bmod 2 \pi
$$

where $C_{f} \geq 1$. Figure 3.3 gives an overview. The borders are emanating from the cusp point $(\mathrm{CU})$, which gives the lower boundary for the coexistence of three modes. Increasing $C_{f}$, additional mode-pairs are created. The coexistence of several modes at distinct feedback phase conditions gives rise to a fold structure, introducing hysteresis for feedback parameter variations.

\subsubsection{Feedback regimes}

The stationary behavior of a laser subject to feedback is strongly dependent on the feedback parameter $C_{f}$. Very different setups can lead to identical values for $C_{f}$, e.g. by having a long feedback section which provides weak feedback or vice versa. With respect to the laser dynamics, an adequate description additionally has to take into account the time constants in the system. In a feedback setup, the characteristic 


\section{Delayed optical feedback}

times are the delay $\tau$ and the period of the relaxation oscillations $\tau_{R O}$. For typical semiconductor lasers $\tau_{R O}$ is in the order of 0.1 to $1 \mathrm{~ns}$. Three regimes can be addressed by changing $\tau$ with the feedback cavity length.

Long cavity regime The situation where the external cavity length is in the order of meters is typically referred to as long cavity regime (LCR). Here, $\tau$ is much larger than $\tau_{R O}$ and the laser interacts with a quasi-continuum of ECMs. Apart from stable cw emission for extremely low $\left(<10^{-8}\right)$ or very strong feedback $\left(>10^{-1}\right)$ [83], dynamical states of high complexity can be observed. Coherence collapse (CC) [65] and low frequency fluctuations (LFF) [37, 78] are the typical phenomena, arising from the interaction of relaxation oscillation dynamics with the mode spectrum. For both, CC and LFF regime, the change of feedback phase does not influence the global dynamics. This is obvious from the irregular switching of the laser between the compound-cavity modes, which makes the definition of a feedback phase useless.

Short cavity regime Very recently, short external cavities $(1-5 \mathrm{~cm})$ have gained interest [45]. In this regime, the delay time is of the same order as the relaxation oscillation period $\left(\tau \approx \tau_{R O}\right)$. As a consequence, only a few external cavity modes participate in the dynamics, reducing the experimental situation to an intermediate complexity compared to the LCR. A characteristic phenomenon in the short cavity regime (SCR) is the occurrence of regular and irregular pulse packages [46]. They originate from the laser mode visiting different ECMs. The most distinguishing feature of the SCR, compared to the long feedback setups, is the sensitivity on the feedback phase. It allows to switch the device dynamics from stationary emission to the generation of regular and irregular pulse packages.

Very short feedback cavities The relaxation oscillation dynamics becomes slow compared to the roundtrip time for very short feedback cavities. Here, the delay section has a similar length as the laser (some $100 \mu \mathrm{m}$ ). Such a setup was first investigated by Tager and Petermann [82]. By numerical and analytical investigations of the LK equations they found parameter combinations, where two longitudinal modes can be adjusted for simultaneous lasing. For the preparation of this experimental situation, the feedback parameter $C_{f}$ has to be chosen according to Fig. 3.3 within a range where three modes can coexist. Due to the short feedback delay high feedback strengths are required. The rest of this thesis is dedicated to the investigation of dynamical phenomena arising in the very short feedback regime.

\subsubsection{Characteristic dynamics in the very short cavity limit}

The dynamics in the very short cavity limit strongly depends on the feedback intensity. For low feedback strengths, the experimental situation is similar to a solitary laser. Hence, the dynamics is governed by the relaxation oscillations, which can be modified by the re-injection of light. Once the feedback is strong enough that a pair of 
longitudinal modes can coexist, additional dynamics is induced by beating phenomena. Both situations are briefly discussed here.

\section{Mode beating}

Mode beating requires two longitudinal modes which are simultaneously lasing. In order to achieve high frequencies and to allow for dynamics at higher frequencies than the ROs, the spacing of the modes has to be large, i.e. a short feedback cavity is required. From the practical point of view integrated devices are preferable. Here, the length of laser and feedback section is in the $100-\mu \mathrm{m}$ range, resulting in a ratio $\tau / \tau_{R O}=0.01$. This corresponds to the configuration first investigated by Tager and Petermann [82]. The most important finding of their work was a stable coexistence of mode and antimode, provided sufficiently strong feedback and an appropriate feedback phase. These results were later confirmed by analytical investigations in Refs. [94, 36]. ${ }^{2}$

The parameter conditions for the coexistence of two longitudinal modes can be derived from Eqs. (3.7). Beating phenomena occur, when two modes have equal threshold gain $G_{1}=G_{2}$, but different lasing frequencies $\Omega_{1} \neq \Omega_{2}$. The beating frequency is defined by the spacing of the compound-cavity modes. Figure 3.2(c) illustrates the threshold gain of modes for a fixed feedback strength $\left(C_{f}=6.6\right)$ and changing phase. Due to the large $C_{f}$ three or five modes fulfill the roundtrip condition. Mode beating can occur for the intersection of stable and unstable branches of the mode equation solutions. An investigation of the mode stability [86] shows that both modes stabilize each other in a small range of parameters. In this way, stable beating is achieved.

A few important results regarding the MB can be obtained analytically from the LK equations $[94,21]$. The relation between feedback strength $K$ and beating frequency $f$ is given by

$$
\begin{aligned}
K & =K_{0}\left|\frac{\pi f \tau}{\sin \pi f \tau}\right|, \quad \text { with } \\
K_{0} & =\frac{q^{\prime} \tau_{l}}{2 \tau} .
\end{aligned}
$$

$K_{0}$ determines the minimum feedback strength necessary for the occurrence of two modes with equal threshold. It is defined by the value of the inverse reflectivity slope $q^{\prime}$ and the roundtrip times in the EC and DFB cavity, $\tau$ and $\tau_{l}$, respectively. Since the feedback strength $K$ is limited to unity (i.e. the full emission is re-injected), a lower boundary for the EC-length is given by the condition $K_{0}<1$.

The minimum feedback strength required for MB pulsations depends on the DFB characteristics in different ways. With $q^{\prime}$ the readjustment of DFB wavelength and threshold due to the feedback is regarded. The value of $q^{\prime}$ increases as a function of $\kappa L$ (see Fig. 2.3). Hence, low $\kappa L$ values reduce $K_{0}$ or increase the resulting frequency for constant $K$. Furthermore, the DFB characteristics enters $K_{0}$ by the effective value

\footnotetext{
${ }^{2}$ For long external feedback cavities situations with coexisting modes having equal threshold gain already exist for low feedback strength. The close spacing of ECMs and fluctuations due to spontaneous emission make these configurations unstable [72].
} 


\section{Delayed optical feedback}

of $\tau_{l}$. In a laser, the dispersion close to the lasing mode strongly depends on the inversion. The dispersion results in an additional phase shift for the propagating light in the laser cavity, which can be accounted for by introducing an effective length of the DFB section $[56,53]$. The effective length can exceed the physical length, leading to an increased $K_{0}$ and lower pulsation frequencies for a given feedback strength $K$. Since an increased effective length results in a lower mode spacing close to the main DFB modes, this effect is called mode pulling. The impact of mode pulling will be experimentally demonstrated in Section 4.2. As a rule of thumb, for high-frequency beating oscillations a short feedback cavity which provides high feedback strength is necessary.

\section{Undamped relaxation oscillations}

Below the feedback strength required for MB only single-mode dynamics occurs. An analysis of the short cavity regime in [94] has shown that the single-mode scenario can give rise to self-sustained pulsations. These oscillations are similar as in multisection lasers comprising two detuned gratings $[8,85]$. The setup in these devices involves a DFB mode positioned spectrally on a decreasing reflectivity slope of the other DFB section. Mediated by $\alpha_{H}$, any fluctuation in the lasing DFB section leads to a variation of the wavelength. In presence of dispersive feedback this induces a change of the feedback strength, i.e. the resonator quality of the cavity is modified. For this reason, the effect is also called dispersive Q-switching (DQS). As a result of DQS, periodic modulations of the laser intensity are observed; frequencies are slightly enhanced compared to the damped ROs of a solitary laser.

A phenomenological explanation for undamped ROs (the term is used synonymous to DQS) can be obtained from modified rate equations for the DFB laser. Taking into account the impact of a dispersive reflectivity [5], one obtains

$$
\frac{d S}{d t}=\left[v_{g} \Gamma(n) g(n, S)-\gamma_{i}-\gamma_{m}(n)+\frac{1}{2} \frac{d}{d t} \ln K_{z}(n)\right] S .
$$

Here, $S$ denotes the photon and $n$ the carrier number. $\Gamma(n)$ describes the fraction of $S$ located in the active section, $g(n, S)$ the material gain in the active section, $v_{g}$ the group velocity, and $\gamma_{i}$ and $\gamma_{m}$ are the internal and the mirror loss rate. The spectral correlation of the lasing mode and the feedback dispersion makes both the mirror loss and the longitudinal excess spontaneous emission factor $K_{z}$ dependent on the carrier density. In the feedback setup with a fixed reflectivity the mirror losses are independent of the carrier density.

A small signal analysis [51] of Eq. (3.10) and the corresponding carrier equation gives a modified resonance frequency $f_{r}$ for the compound cavity

$$
f_{r}=\frac{1}{2 \pi} \sqrt{\left(\gamma_{m}+\gamma_{i}\right) S \frac{\partial}{\partial n}\left(v_{g} \Gamma g-\gamma_{m}\right)} .
$$

It is obvious that the carrier dependence of the longitudinal confinement factor and mirror loss can either enhance or decrease the resonance frequency. In case of a decreasing reflectivity slope the effective differential gain and the resonance frequency 
increase. The stability of the mode is described by its damping rate

$$
\Gamma_{r}=\frac{1}{\tau_{d}}+\left[v_{g} \frac{\partial(\Gamma g)}{\partial n}-v_{g} \Gamma \frac{\partial g}{\partial S}+\frac{1}{2}\left(\gamma_{m}+\gamma_{i}\right) \frac{\partial \ln \left(K_{z}\right)}{\partial n}\right] S
$$

with the differential carrier lifetime $\tau_{d}$. Again, the damping decreases for a negative reflectivity slope due to the last term in this equation. This explains that ROs can be undamped and self-sustained pulsations occur. Additionally, the RO frequency is enhanced by an increase of the effective differential gain. In the investigated setup of a laser with short feedback the external cavity itself acts as dispersive reflector.

In summary, two different pulsation types can be expected for a very short delayed feedback setup. At low feedback strength undamped relaxation oscillations occur. High feedback intensity causes beating phenomena.

\subsection{Experimental verification: the passive feedback laser}

This Section gives an experimental verification of feedback phenomena in the short cavity limit. Although the first numerical investigation of MB pulsations in this regime dates back to 1994 [82], a practical demonstration was lacking due to experimental difficulties. The problems arise from the length of the feedback cavity in the sub-mm range. An appropriate setup has to provide the control over the feedback phase, i.e. the length of the feedback cavity has to be adjustable in the sub-wavelength domain. Additionally, high feedback strengths are necessary to achieve MB pulsations. This requires monolithically integrated devices.

\subsubsection{Device}

A passive feedback laser (PFL) was realized to verify the predicted dynamical phenomena for very short feedback cavities. Figure 3.4 shows a schematic of the device. The

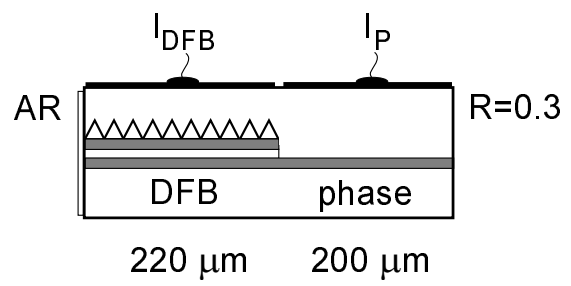

Figure 3.4: Schematic of realized PFL. A passive feedback section is monolithically integrated with a DFB laser. A cleaved facet acts as mirror.

DFB section length is $220 \mu \mathrm{m}$, with a coupling coefficient of $\kappa=130 \mathrm{~cm}^{-1}$. Integrated with the DFB laser is a $200 \mu \mathrm{m}$ long passive delay section. The DFB section facet is 


\section{Delayed optical feedback}

anti-reflection $(\mathrm{AR})$ coated, while a cleaved facet with a power reflectivity of $R \approx 0.3$ acts as mirror. Details on the device realization will be summarized in Section 4.1. Using the injection of free carriers into the passive section [14], the refractive index and hence the feedback phase can be tuned. The delay time $\tau$ is not affected measurably by a change of injection current nor by a slight change of lasing wavelength due to a shift in laser threshold. Due to free-carrier absorption any change of feedback phase induces a modification of feedback strength. Although both feedback parameters in the PFL setup can be changed by current injection, the range of accessible feedback strengths for a given facet reflectivity is limited. Strong variations of $K$ require the modification of the device, e.g. by using a highly reflective coating on the mirror facet.

\subsubsection{Overview on dynamics}

An extensive investigation of the PFL has been presented by Ushakov et al. in [86]. For the sake of completeness and to show the validity of the previous considerations, an overview on the device dynamics is presented here. Figure 3.5 shows pulsation frequencies in the parameter plane of phase and DFB current. ${ }^{3}$ The white areas indicate

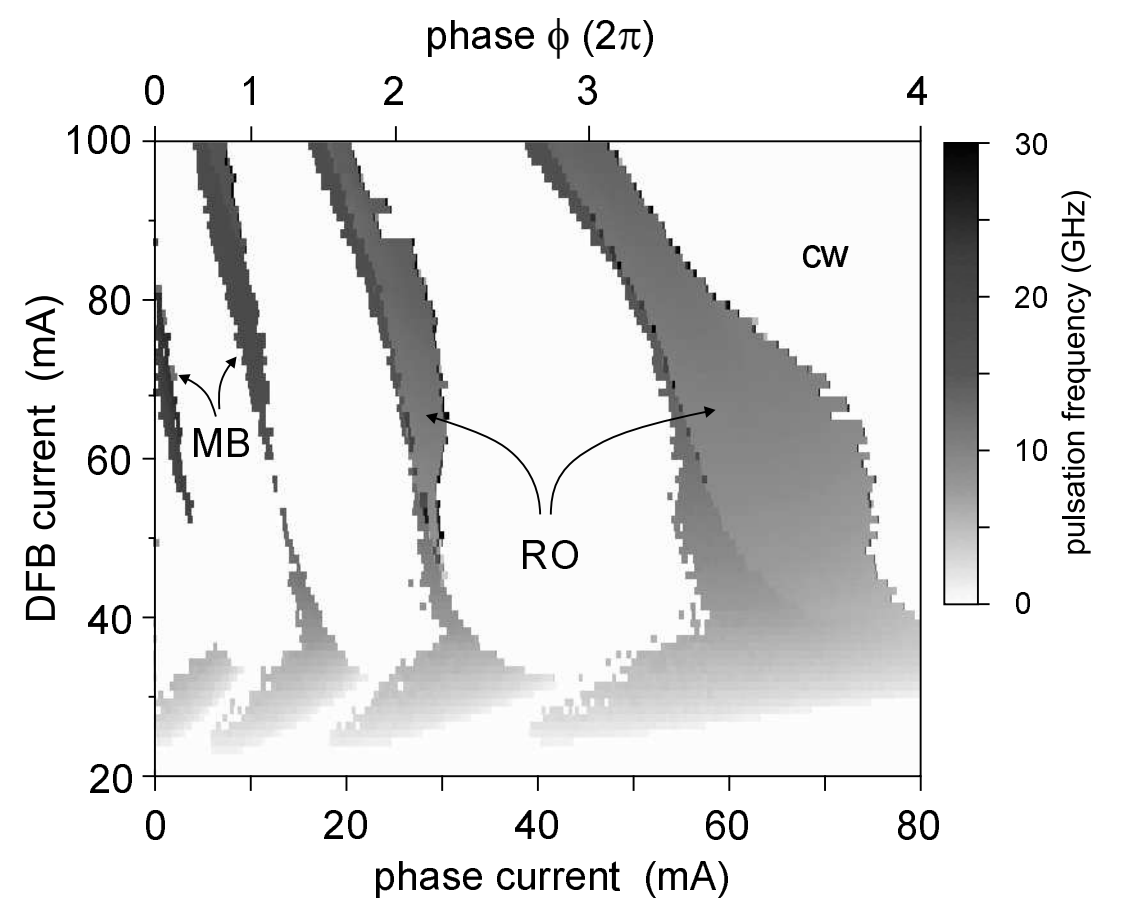

Figure 3.5: Overview on PFL dynamics. Pulsation islands are recurring with $I_{P}$, frequencies vary due to changing feedback strength. Besides the large areas of cw output, MB and undamped RO pulsations are observed.

operation points of $\mathrm{cw}$ emission or with an rf power less than $5 \mathrm{~dB}$ above the noise-floor of the electrical spectrum-analyzer (ESA). Four distinct islands can be addressed by the

\footnotetext{
${ }^{3}$ Measurement setup was identical to the investigations in Chapter 4.4.2.
} 
index change in the passive section. The characteristics of the pulsations differs from island to island. One reason is the decreasing phase shift efficiency, which broadens the pulsation areas. Additionally, the feedback strength decreases with increasing $I_{P}$. Hence, the MB-type pulsations gradually change into ROs. The DFB current has a minor impact on the basic device behavior. It shifts the DFB wavelength due to thermal effects and causes a tilting of the pulsation islands. Additionally, an enhancement of the pulsation frequency due to an increased photon density is observed for the undamped RO pulsations.

The investigations of the short feedback cavity regime to this point have shown that although providing only one or two lasing modes, various dynamics can be prepared. For an experimental investigation integrated devices are required, which employ freecarrier absorption for phase tuning. A drawback of integrated phase tuning sections is the lack of an efficient control for the feedback strength. Changes in $K$ beyond the range accessible by the excess-loss due to free-carrier absorption requires a modification of the device, i.e. the reflectivity of the feedback section. An analysis of the whole $(\phi, K)$ parameter range is therefore not possible with a single PFL.

With respect to the technical application, these results clearly show that the initial aim of beating frequencies considerably exceeding the frequency of RO pulsations has not been achieved. The reason is the limited amount of feedback. A possible improvement enabling higher pulsation frequencies is a high-reflection $(R>0.99)$ coating for the rear facet of the device.

These limitations were the reason that the PFLs were not further investigated in the framework of this thesis. The devices were transferred to Humboldt-University for a detailed experimental analysis. Results regarding different dynamical scenarios can be found in Refs. [86, 15, 96]. A modified device concept was developed for further studies of the characteristic dynamics. It provides the access to the same experimental scenario as the PFL, however, with independent control of feedback strength and phase. 


\section{Chapter 4}

\section{The concept of active feedback lasers}

In this Chapter the active feedback laser (AFL) is introduced as new laser type. It integrates an active feedback section, which allows the separate control of feedback phase and strength. An experimental overview on the dynamics of a realized AFL verifies the basic considerations underlying its design. Simulations substantiate that the investigated device exhibits characteristic behavior.

\subsection{Idea and realization}

Figure 4.1 sketches an AFL [11]. The basic setup is similar to the PFL, however, the feedback cavity is implemented as a combination of passive and amplifier section. This allows for a separate control of feedback phase (phase section) and feedback strength (amplifier section) using current injection. Strong feedback is adjustable since the

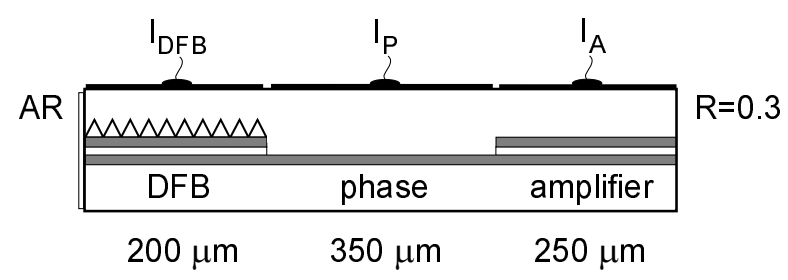

Figure 4.1: Schematic of an AFL. An additional amplifier section enables the control of feedback strength. The different sections are controlled by independent currents.

amplifier can compensate for losses arising in the device (i.e. in the phase tuning section, at the section interfaces, and the amplifier facet). Moreover, the gain in the amplifier section can increase the overall internal photon density, producing new effects in comparison to passive feedback. The dynamics of the additional carrier density in the amplifier will turn out as important for the device dynamics.

An AFL has been fabricated for experimental investigations. Section lengths are $200 \mu \mathrm{m}, 350 \mu \mathrm{m}$, and $250 \mu \mathrm{m}$ for laser, phase, and amplifier section, respectively. The 
employed hetero-structure is equivalent to the DFB lasers described in Chapter 2. Main parameters are a Bragg-wavelength of $1539 \mathrm{~nm}$ and a coupling coefficient $\kappa=130 \mathrm{~cm}^{-1}$. The front facet of the device is AR coated.

The phase section is realized by removing the active layer and the upper waveguide of the DFB structure. The $1.3 \mu \mathrm{m}$ waveguide layer is directly overgrown with $\mathrm{p}^{+}-\mathrm{InP}$ and structured with a $2.4 \mu \mathrm{m}$ ridge for lateral waveguiding. Active and passive sections are electrically isolated (typical resistance between sections $5 \mathrm{k} \Omega$ ) and can be separately biased. The interface between the sections is tilted by $10^{\circ}$ in order to suppress residual reflections. The single-pass power loss at the interface amounts to $10 \%$, in addition to waveguide losses of $\alpha_{P}=8 \mathrm{~cm}^{-1}$ for the passive section [17].

The amplifier comprises the same transversal structure as the DFB section, only the grating in the upper waveguide is omitted. The amplifier facet remains as cleaved with a power reflectivity of $R \approx 0.3$. In this way, it acts as mirror, but preserves the possibility to measure at this facet. The total length of the integrated feedback section is chosen in order to provide $40 \mathrm{GHz} \mathrm{MB}$ pulsations. Thus, the interesting dynamics lies in a frequency range accessible for available spectrum analyzers, while MB and RO pulsations act on different time scales. A numerical investigation of the relation between feedback cavity length and upper frequency limit is given in [9].

Figure 4.2(a) shows a multi-section laser mounted on a heatsink. Both facets are coupled to an optical fiber, the electrical connections are given by bond-wires. Additionally, the heatsink acts as ground contact. The whole setup is mounted in a module, providing easy handling and the long-term stability of fiber/chip coupling (Fig. 4.2(b)). A temperature sensor and a Peltier element are integrated into the module for stabilizing the laser temperature.

(a)

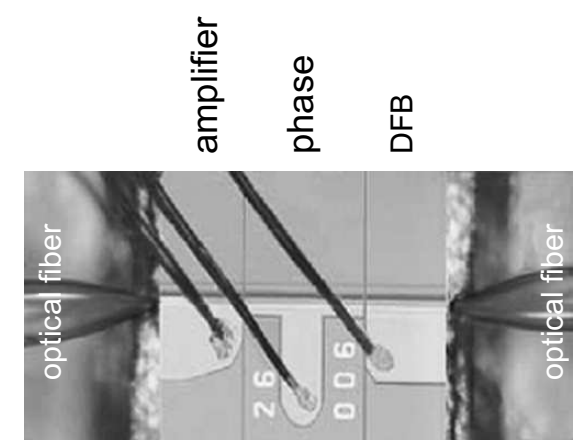

(b)

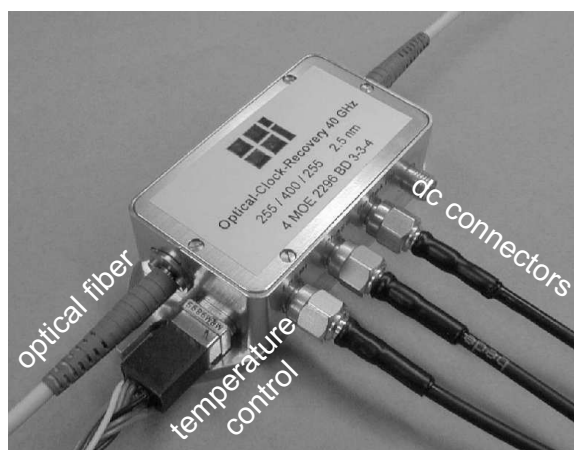

Figure 4.2: Self-pulsating lasers. (a) The multi-section laser chip mounted on a heatsink, optical fibers and electrical connections are shown. (b) Module comprising a multi-section laser. 


\subsection{Integrated feedback cavity characteristics}

\section{Adjusting the feedback cavity length}

When designing an AFL, the first consideration regards the length of the feedback cavity, because it has to be defined in the fabrication process and remains fixed afterwards. Besides controlling the maximum number of coexisting modes, the feedback cavity length can additionally influence the selection between longitudinal modes.

In the solitary DFB laser, longitudinal spatial hole burning (LSHB) provides a gain margin which stabilizes the short-wavelength mode. However, feedback modifies the threshold of longitudinal modes due to different feedback phases. Once the gain-margin by LSHB becomes smaller than the feedback-induced gain shift, mode switching occurs when the feedback phase is varied. To counteract this effect, the feedback cavity length has to be adjusted to the DFB characteristics. The aim in cavity design is to provide similar feedback phase conditions for both stop-band modes. Hence, their threshold is modified in the same way when the phase changes (i.e. they move along the ellipses on the same position). Given such a configuration, already a small gain margin due to LSHB is sufficient for stable emission on the short wavelength mode. The relative phase $\Delta \phi$ for the two stop-band modes is defined by

$$
\Delta \phi=4 \pi n_{e f f} L_{E C}\left(\frac{1}{\lambda_{\mathrm{TE}_{-1}}}-\frac{1}{\lambda_{\mathrm{TE}_{+1}}}\right) .
$$

It therefore depends on the length of the feedback cavity $L_{E C}$ and the refractive index $n_{e f f}$, as well as from the value of $\kappa L$, which influences the wavelengths $\lambda_{\mathrm{TE}_{-1}, \mathrm{TE}_{+1}}$ of the dominant modes. A relative phase shift of $\Delta \phi=\pi$ requires $\sim 40 \mu \mathrm{m}$ variation of the EC-length, assuming the typical laser parameters of the investigated devices. Therefore, these modifications imply only minor changes of the device geometry. Given a proper EC-length, additional technological modifications for mode selection, e.g. by an inhomogeneous grating in the DFB section [50], are dispensable.

\section{Phase and amplitude characteristics}

After realizing an AFL with appropriate feedback cavity parameters, the first steps in characterization regard the feedback phase and strength. In an AFL, the feedback cavity characteristics can be determined for an individual multi-section device, instead of relying on results obtained from test-structures. The measurements are enabled by the amplifier in the feedback cavity.

Optical spectra measured from the amplifier facet are shown in Fig. 4.3. DFB and amplifier current were adjusted to $20 \mathrm{~mA}$ and $45 \mathrm{~mA}$, respectively. The phase section bias is varied in order to investigate the dependence of the feedback phase from the injected current. The region of high ASE modulation-depth indicates the DFB stopband. Within the stop-band, the intensity modulation is defined by the geometrical EC-length plus the penetration depth of the light into the grating. The $0.5 \mathrm{~nm}$ ripplespacing at the stop-band center corresponds to an effective length of $650 \mu \mathrm{m}$ (i.e. $50 \mu \mathrm{m}$ penetration depth into the DFB section). At the edges of the stop-band, mode pulling 


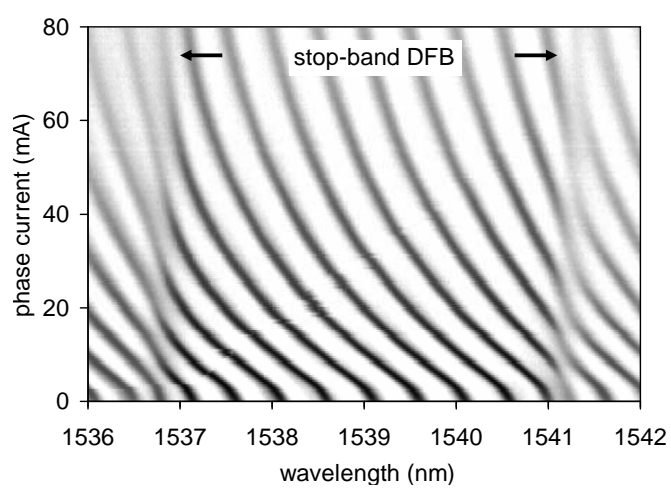

Figure 4.3: Optical spectra measured from the amplifier facet for changing $I_{P}$. Intensity is encoded in gray scale (log-scale).

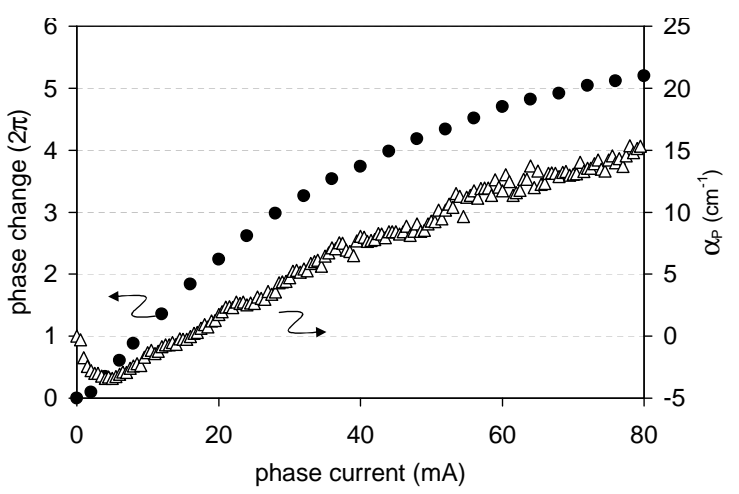

Figure 4.4: Dependence of feedback phase $\phi_{P}$ and excess loss $\alpha_{P}$ from $I_{P}$.

reduces the spacing of modes to approximately $0.3 \mathrm{~nm}$, corresponding to an effective length of $1070 \mathrm{~nm}$. This gives a first indication that the upper frequency limit for the investigated device will be in the range of $40 \mathrm{GHz}$.

From the wavelength shift of ASE modulation the current dependency $\phi_{P}\left(I_{P}\right)$ can be determined (Fig. 4.4). A change of feedback phase by five periods is observed, before heating-effects counteract the carrier-induced phase shift. An analysis of the ASE modulation results in an excess loss of $4 \mathrm{~cm}^{-1} / 2 \pi$. This is in good agreement to the values reported for the PFL, which were determined from test-structures [86]. The origin of the decreasing loss within the first $5 \mathrm{~mA}$ is not clear. A possible reason could be leakage currents from the passive to the active sections, which unintentionally increase the gain. However, the resistance between passive and active sections is $5 \mathrm{k} \Omega$, thus excluding currents which could explain the observed decrease in losses. Measurements with the DFB section pumped at transparency showed the same effect. Thus, the decrease of losses is not related to a change in DFB reflectivity due to gain or absorption.

The second important characteristics of the feedback cavity is the dependence of feedback strength on the amplifier current $I_{A}$. It is possible to determine the gaincurrent characteristics of the amplification from the ASE modulation depth. The DFB section has to be pumped at its material transparency level to suppress the interaction with the feedback cavity light. A reference point in the determination of the absolute $K$-value is given by the lasing threshold of the feedback cavity modes. From Eq. (3.4) it is known that feedback cavity modes at threshold have to fulfill the condition of unity roundtrip gain, i.e. the relation $q=K$ holds. The inverse DFB reflectivity $q$ at transparency can be determined from simulations. Additionally, losses at the DFB/feedback cavity interface have to be taken into account. The full $K\left(I_{A}\right)$ characteristics below threshold is then easily obtained by scaling the threshold feedback strength $K_{t h}$ with the gain-current characteristics of the ASE. The drawback of this straightforward method is that it can only be used for the unsaturated feedback characteristics $K_{\text {unsat }}\left(I_{A}\right)$. As soon as the DFB section starts lasing, the high photon density induces gain saturation in the amplifier, resulting in a saturated feedback strength $K_{\text {sat }}<K_{\text {unsat }}$. 
A further complication in determining the feedback strength arises from the coupling of the carriers in the amplifier section to the overall photon density. The carrier density - and therefore the gain - is no longer governed by the injection into the amplifier section only. Instead, it becomes dependent on the lasing state of the AFL. Later investigations will show that the dependence of feedback parameters on the lasing state is responsible for stabilizing effects in the AFL dynamics.

\subsection{Optical modes: Impact of strong feedback}

The higher feedback level enabled by the active section in the AFL makes it worthwhile to redo the mode analysis presented in Section 3.1. Position and the respective damping for the dominant modes are investigated using the parameters given in Appendix A.1. The approach is similar as for low feedback strength $K$, now taking into account the large range for $K$ by solving the full characteristic Eq. (3.4). $K$ is directly controlled by the gain in the amplifier section, which depends on the averaged carrier density $\bar{n}_{A}$. For the mode analysis the carrier density in the DFB section is kept fixed at the solitary laser threshold.
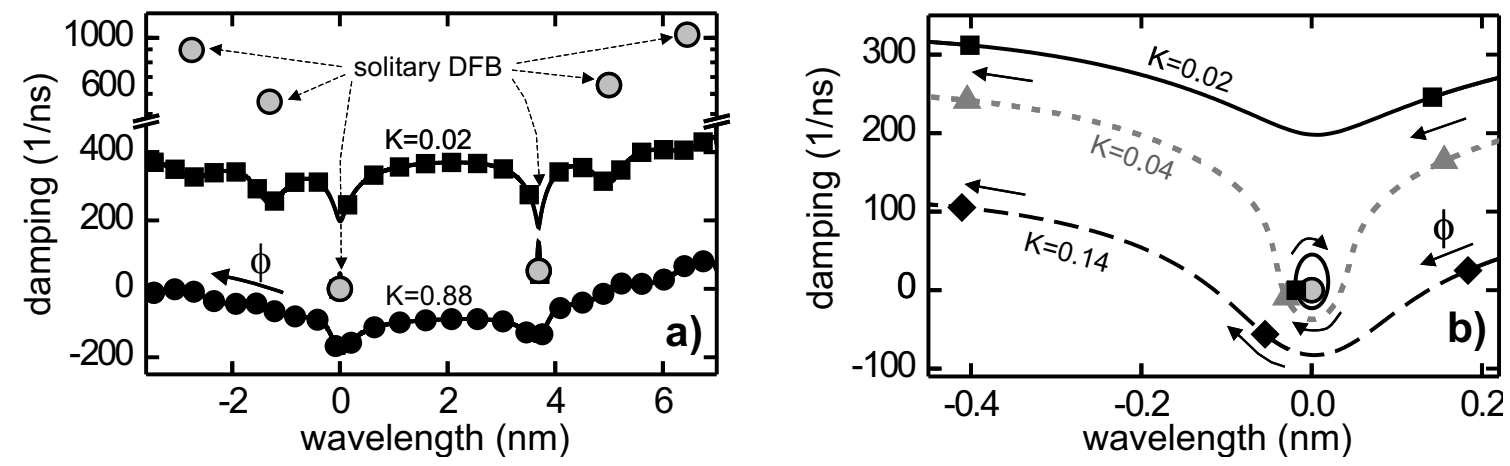

Figure 4.5: Spectrum of optical modes for the investigated AFL geometry. (a) Overview; (b) environment of the lasing mode. Bullets: solitary laser $(K=0)$. Full symbols: $K=$ $0.02,0.04,0.14$ and 0.88 , calculated under following assumptions: no gain saturation, no LSHB, feedback phase fixed. Lines: locations of modes for all possible feedback phases $\phi$. Arrows: direction of increasing $\phi$. After one phase period each mode replaces its formerly next neighbor on the same line.

Figure 4.5(a) illustrates the change of the mode spectrum for different feedback strengths $K$. Three distinct areas occur. Outside the stop-band of the DFB, the mode spacing is $0.4 \mathrm{~nm}$ and defined by the total length $(800 \mu \mathrm{m})$ of the device. Within the stop-band, a FP-like cavity is formed by the DFB grating on the one side and the amplifier reflectivity on the other. The shorter cavity length results in a mode spacing of $0.5 \mathrm{~nm}$. Mode pulling occurs from the excess phase shift of the laser section at the stop-band edges. The spacing of the compound-cavity modes is almost independent of the feedback strength $K$. These observations are in full agreement to the measurements presented in Fig. 4.3. 
While the mode positions remain unchanged, the damping of the FP-type modes is strongly affected by $K$. For $K=0.02$, corresponding to $I_{A} \approx 0 \mathrm{~mA}^{1}$, the decay times are shorter than 5 ps and therefore these modes are not essential for the device dynamics. However, the mode damping is strongly reduced with increasing amplification. Negative values, as occurring for the highest $K$-value, are an artifact due to the fixed carrier density $n$ in the DFB section. If this constraint is relaxed, $n$ will adjust until the damping of the lowest-loss mode reaches a minimum value of zero.

Besides introducing additional modes, the feedback modifies the lasing mode as shown in Fig. 4.5(b). For low feedback, the impact is weak. When tuning the feedback phase by one period, the lasing mode moves around an ellipse in the dampingwavelength plane. The behavior is analogous to results obtained by the linear approximation Eq. (3.6) of mode positions close to the DFB resonance. Additional FP-type modes move on a separate nonclosed line, replacing the formerly next neighbor after one period. Single-mode dynamics is expected in this regime. With increasing feedback, the ellipse blows up until it touches the FP-like branch and merges with it. Similar results were described for a double-cavity laser in Ref. [30]. Beyond this feedback strength all modes are located on a single open line, exhibiting a deep valley close to the wavelength of the solitary laser. When increasing feedback phase, the modes move from right to the left through this valley and take over lasing as long as they are the one of lowest damping. This repeats with a period of $2 \pi$, always with a new mode. As the new mode moves down, its predecessor climbs up on the opposite side of the valley. In a certain phase range, both modes have comparable damping and contribute to the laser dynamics. Stronger amplification, i.e. feedback, makes the valley less distinct, so that even more than two modes can come into play. Regions of coexisting MB and RO pulsations can arise for a situation where two modes stabilize at threshold, but start to oscillate around their stable position.

\subsection{Experimental exploration of device dynamics}

\subsubsection{Measurement setup}

One basic measurement setup has been employed for the characterization of the AFL dynamics throughout this work. It consists of

- the device under test,

- current supplies and a temperature control for the device,

- and measurement equipment for characterizing the optical output of the laser.

An additional optical pulse source is used for investigations which require external perturbations. The whole measurement setup is controlled with a computer.

\footnotetext{
${ }^{1}$ Absorption in the amplifier generates carriers and decreases losses, i.e. $K \neq 0$ for $I_{A}=0$.
} 


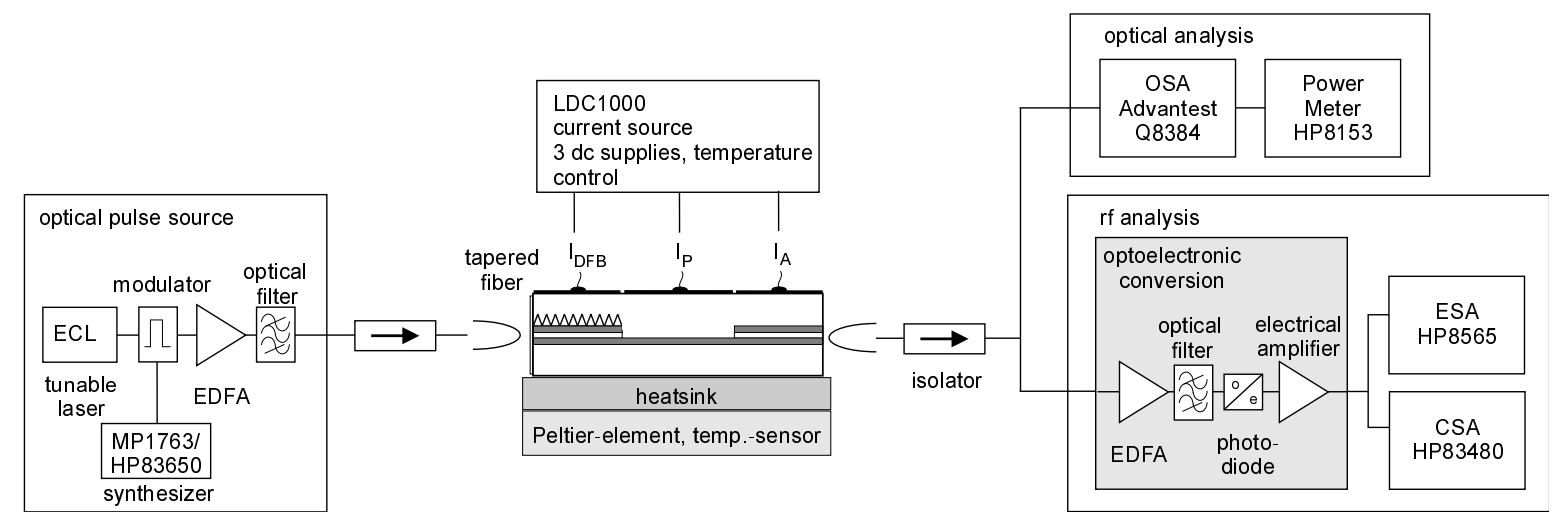

Figure 4.6: Diagram of the measurement setup. The main building blocks are: the investigated laser, a current supply, devices for characterizing the optical signal, and an optical pulse source.

All analyzed devices are packaged into modules, which provide a stable coupling between laser and optical fiber, shield the laser from electromagnetic perturbations, and allow for a constant device temperature. Perturbations of the laser by spurious back-reflections from the measurement setup are prevented by an optical isolator. The laser sections are driven with a constant current provided by a Profile LDC1000. This unit also includes a temperature control to stabilize the device temperature at $20^{\circ} \mathrm{C}$ $\left( \pm 0.01^{\circ} \mathrm{C}\right)$.

Several instruments are applied for the measurements. An optical power meter (Hewlett-Packard HP8153A) is used for the determination of the total optical power coupled into the fiber. Optical spectra are recorded with an Advantest Q8384A optical spectrum analyzer (OSA) providing a minimum resolution bandwidth of $0.01 \mathrm{~nm}$. The dynamics of the optical output is analyzed by an electrical spectrum analyzer (ESA) HP8565E after converting the optical signal into the electrical domain by a u2t photodiode. ESA and photodiode provide a bandwidth of $50 \mathrm{GHz}$. With an electrical amplifier (HP83050A) the electrical rf signal can be boosted in order to improve the signal-to-noise ratio at the ESA. Additionally, the optical signal can be amplified by an erbium doped fiber amplifier (EDFA). Noise added by the EDFA is removed with a subsequent optical bandpass filter (bandwidth 1-3 nm). The temporal characteristics can be analyzed by a sampling oscilloscope HP83480 (50 GHz bandwidth). An optical pulse source is employed for synchronization and response measurements. It consists of an external cavity laser (ECL, Photonetics Tunics) which provides a tunable lasing wavelength with a narrow linewidth (typically $10 \mathrm{kHz}$ ). An electro-absorption modulator (OKI EAM OM5642W-30B) is used to generate optical signals with frequencies up to $50 \mathrm{GHz}$. It is either driven by a pulse pattern generator (Anritsu MP1763) or directly by an electrical synthesizer (HP83650). The complete measurement setup is sketched in Fig. 4.6. Modifications of this setup are denoted in the text. 
The AFL provides three control currents to adjust the device behavior. Since already minor changes of a single parameter can induce strong variations in the dynamics, a complete characterization requires a large number of measurements. To enable comprehensive investigations, a standardized measurement routine has been implemented in a LabVIEW ${ }^{\mathrm{TM}}$ program. It varies the injection currents in three nested loops. At each current combination the optical peak-power and wavelength, as well as the rf peak-power and frequency are recorded. Additionally, optical and electrical spectra can be saved in predefined steps. To achieve reasonable measurement times, the AFL behavior is typically investigated with respect to the feedback parameters only.

\subsubsection{Overview}

After having verified the functionality of the feedback sections, a characterization of the overall dynamics can proof the basic considerations underlying the AFL device. In contrast to the PFL, there are two external control parameters for the feedback: the phase and the amplifier current. Accordingly, the device behavior is characterized in the plane given by the feedback parameters $I_{P}$ and $I_{A}, I_{D F B}$ is kept fixed at $70 \mathrm{~mA}$. The DFB mode at the left stop-band side remains dominant within the investigated parameter range. ${ }^{2}$ Similar to the PFL, a change in the DFB injection modifies the device behavior only slightly. An investigation of the DFB current as parameter is given in Appendix A.4.

Measurements were done using the setup described in Section 4.4.1. Main parameter is the phase current, which is varied from $0 \ldots 50 \mathrm{~mA}$ (inner loop). After $I_{P}$ has reached $50 \mathrm{~mA}$, it is reset and the amplifier current is increased. When changing the feedback parameters, the laser output undergoes various transitions from steady-state to self-pulsation, or between self-pulsations of different frequencies. Figure 4.7 gives an overview on the pulsation frequency (only the main peak of the rf spectrum is indicated, ESA resolution bandwidth $(\mathrm{RBw}) 1 \mathrm{MHz}, 1 \mathrm{~mW}$ optical power at photodiode, electrical post-amplification). Several islands of similar behavior are detected. Each island corresponds to a distinct mode or mode-pair and shows its behavior in dependence of the feedback parameters. Only phenomena arising in measurement direction are detected; they may be modified due to hysteresis.

The location of mode-pairs is defined by the refractive index change in the feedback cavity. Along an island, the total change of feedback phase remains within $2 \pi$. The tilt of the islands indicates the amplifier contribution to the feedback phase. It is negative for low amplifier current, where the refractive index is decreased by the growing inversion; the inversion saturates beyond $I_{A}=80 \mathrm{~mA}$. Thermal effects due to current heating result in a reversed tilting of the islands. A comparison among the different mode-pairs shows that the behavior is not fully periodic. Phase shift efficiency depends nonlinearly on the injection, therefore larger current variations are required at high injection levels (cf. Fig. 4.4). The excess loss from injected carriers in the phase section

\footnotetext{
${ }^{2}$ Relating the DFB injection to a threshold current is not reasonable. The threshold of the AFL without injection into the amplifier section is $I_{D F B}=42 \mathrm{~mA}$. It decreases to $20 \mathrm{~mA}$ for $I_{A}=100 \mathrm{~mA}$.
} 


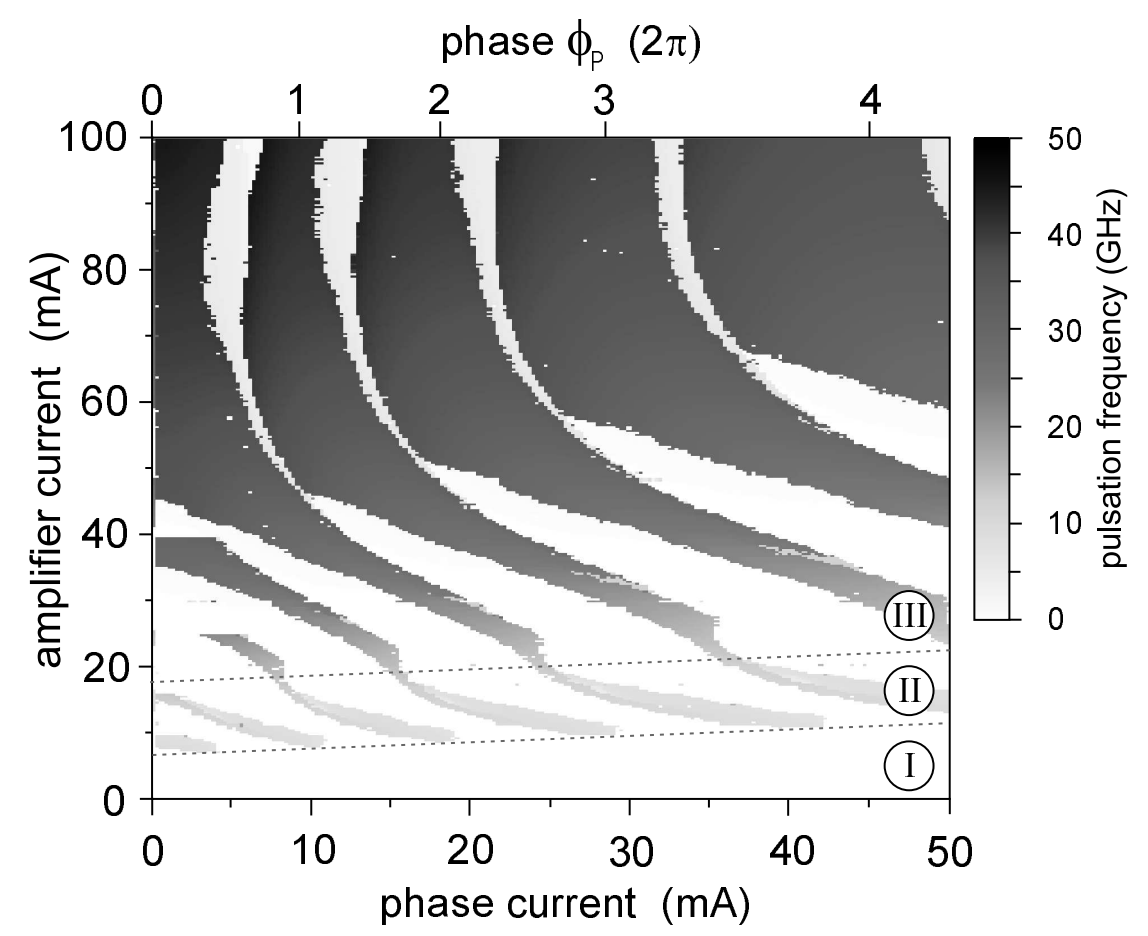

Figure 4.7: Overview on AFL dynamics. Rf power of indicated points is more than $10 \mathrm{~dB}$ above ESA noise-floor. Only the dominant spectral component is indicated. The areas of predominant cw emission (I), RO- (II) and MB- (III) dynamics are labeled.

causes an offset to higher amplifier currents, where the additional gain compensates for the phase section losses.

The observed dynamics can be classified into three regimes. For very low feedback (regime I, amplifier current $<8 \mathrm{~mA}$ in the first period of $\phi_{P}$ ) only cw emission is observed. Undamped relaxation oscillations occur in regime II between $8 \mathrm{~mA}$ and $17 \mathrm{~mA}$. Pulsations with frequencies in the order of $10 \mathrm{GHz}$ can be measured, independent of the feedback strength. The extension of the islands with respect to feedback phase is comparable to a PFL ( $\sim 0.2$ of a period) in the $\mathrm{RO}$ regime. Increasing the feedback strength $K$, the pulsations undergo a transition to MB (regime III). Here, the pulsation frequency is distinctly higher and scales with $K$. In comparison to results for passive feedback, shown in Fig. 3.5, the width of the MB regime in the AFL is increased, significantly exceeding the width of RO pulsations. The islands of MB pulsations widen with increasing feedback strength, until the islands coalesce and pulsating laser output is observed for any feedback phase. Additional ROs can dominate the behavior at high amplifier injection. This is indicated by the light gray regions in between the MB areas in the measurement overview. Further details observed in this overview will be discussed when the respective features are investigated in more detail.

In conclusion, the overview on the dynamical regimes shows behavior already known from the PFL, but modified in some respects. Regimes I and II in the AFL are similar to a PFL which covers the same range of feedback strength. The limited dynamics of the 
amplifier section close to transparency gives only minor modifications. Accordingly, the extension of the undamped RO area is comparable to the PFL, apart from the tilt due to the index change introduced by the amplifier section. Once the amplifier is pumped sufficiently above the transparency level (regime III), it contributes significantly to the device dynamics. An obvious modification is the large extension of MB areas.

\subsection{Numerical simulation results}

Studying the nonlinear behavior of a complex system in experiment, one has to cope with the fundamental problem of identifying generic behavior. A possible approach to separate it from phenomena occurring only in an individual device is the investigation of a large number of identical lasers. Due to the experimental complexity this is not practicable for the present system. Instead, numerical simulations using LDSL were employed to confirm the measurement results.

The simulation of the AFL device is straightforward. A phase section of length $L_{P}$ enters the TWEs by a modified propagation constant

$$
\beta_{P}=-\frac{\phi_{P}}{2 L_{P}}
$$

where $\phi_{P}$ denotes the phase shift in the passive section. For the amplifier section, a coupling coefficient $\kappa=0 \mathrm{~cm}^{-1}$ and boundary conditions accounting for the end-facet reflectivity $R$ are a proper description. Laser parameters for all sections are summarized in Appendix A.1. In analogy to the experimental procedure, the injection into the DFB section is fixed to $70 \mathrm{~mA}$. Losses in the phase section are assumed to $\alpha_{P}=20 \mathrm{~cm}^{-1}$, i.e. slightly higher than the measured values $\left(8 \mathrm{~cm}^{-1}\right)$. In this way, the loss at the section interfaces is taken into account ( $\mathrm{DFB} \leftrightarrow \mathrm{P}, \mathrm{P} \leftrightarrow \mathrm{A})$. Additionally, the current dependency of phase section losses is regarded by $\alpha_{P}=20 \mathrm{~cm}^{-1}+5 \mathrm{~cm}^{-1}\left(\phi_{P} / 2 \pi\right)$.

The carrier-induced change of the gain is by far dominant in the amplifier section and the modification of background losses $\alpha_{A}$ can be ignored. However, current heating alters the background detuning. Following Ref. [8] it is taken into account by assuming $\delta_{A}=\beta_{c} I_{A} / L_{A}$ with the thermal detuning coefficient $\beta_{c}=40 \mathrm{~A}^{-1}$ and the amplifier section length $L_{A}$. In addition to the laser output the feedback strength $K$ is computed as

$$
K=\sqrt{R} e^{-\alpha_{P} L_{P}+\left(g_{A}-\alpha_{A}\right) L_{A}},
$$

where $g_{A}$ is taken at the average density $\bar{n}_{A}$. The upper limit for $K$ is thus defined by the onset of gain saturation. For regular pulsations, the value of $K$ is determined by averaging over one pulsation period.

Figure 4.8 summarizes the numerical results in a contour plot showing the dependency of pulsation frequency from the feedback parameters. The very good agreement with the experimental results (Fig. 4.7) is evident. The different dynamical regimes observed in the experiment are confirmed. Deviations are mainly caused by uncertainties concerning the translation of experimental currents into more fundamental parameters such as feedback strength. The shift of the whole plot to lower amplifier currents is 


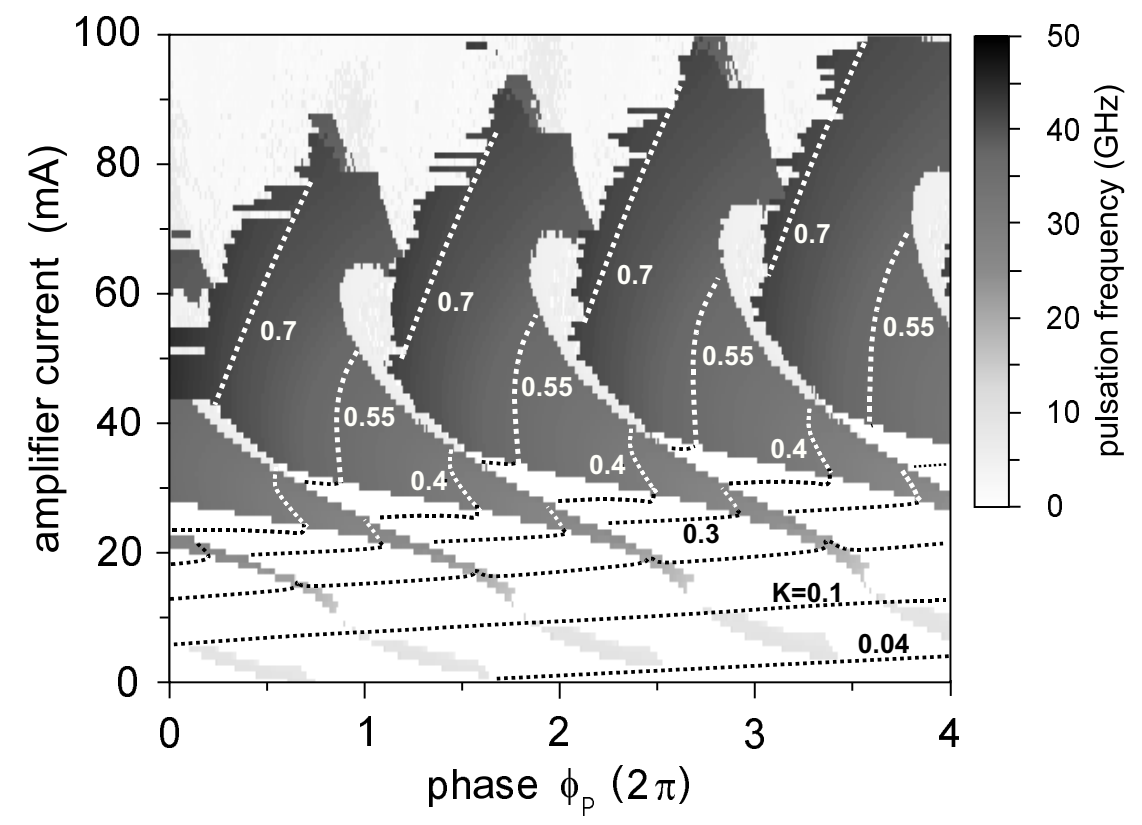

Figure 4.8: Calculated areas of self-pulsation in the plane of $\phi_{P}$ and $I_{A}$. White areas indicate cw emission, gray areas non-stationary laser output. Dotted lines represent curves of constant $K$ (simulations: M. Radziunas [12]).

attributed to underestimated losses in simulation. Borders between the regimes I, II, and III can be determined to $K=0.04$ and 0.12 , respectively. Due to the higher feedback strength in simulation, an additional regime IV arises above $I_{A}=70 \mathrm{~mA}$. Here, undamped ROs again dominate the device behavior. The agreement between simulation and experiment confirms that the experimentally demonstrated dynamics can be regarded as generic for AFL devices.

In order to get a clearer relation between $I_{A}$ and the pulsation frequency, both experimental and numerical results have been re-plotted for a single mode-pair in Fig. 4.9. Neglecting differences in the laser parameters, both data sets display an equivalent evolution of dynamical scenarios with feedback strength. Above the undamped RO region, the increase of the MB pulsation frequency with the amplifier current is obvious. Two observations in the high injection regime are important for the further investigations.

First, the frequency of MB pulsations changes when the amplifier injection is fixed and feedback phase is tuned. This results in the vertical width of the pulsation island. Starting from the onset of MB pulsations, there is a significant increase of the tuning range by feedback phase at higher injection into the amplifier section. The frequency change becomes by far stronger than expected from the loss variation within one period of feedback phase. Reason for this behavior is the decoupling of $K$ from the control parameter $I_{A}$ in the range where MB occurs. The almost vertical alignment of constant$K$ lines in Fig. 4.8 confirms these considerations.

Second, MB and RO oscillations can coexist for strong injection into the amplifier section. These situations arise, when the stable configuration of beating modes is su- 


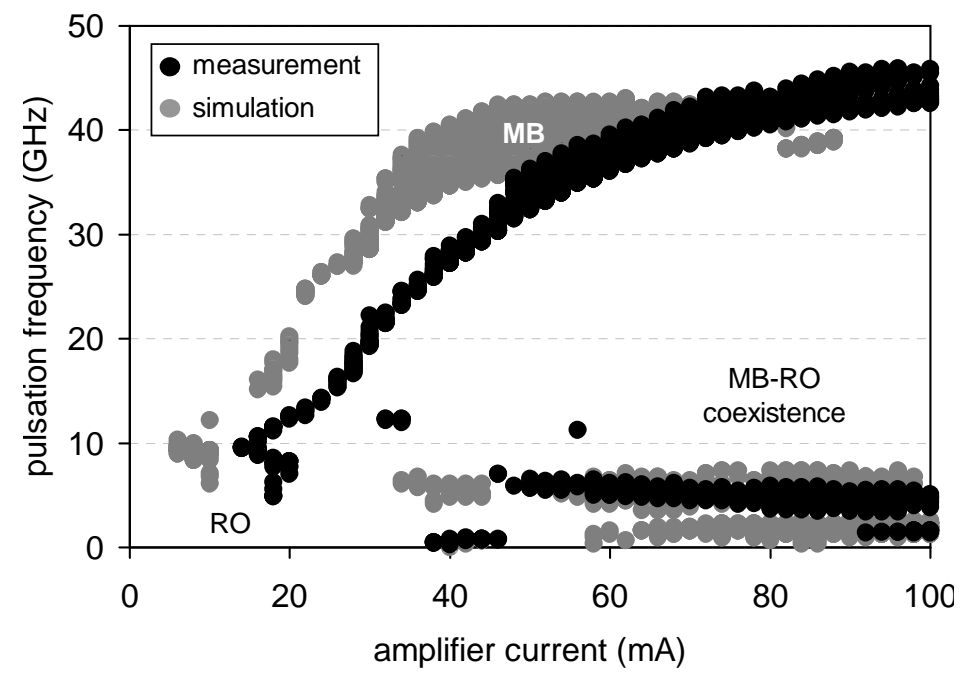

Figure 4.9: Pulsation frequency in dependence of amplifier current. The plot summarizes data from Fig. 4.7 and Fig. 4.8 along a single mode-pair.

perposed by additional relaxation oscillations. Both carrier densities oscillate around their average value while maintaining the MB condition. The modulation of device output due to the carrier density oscillations can become dominant over the MB intensity or even lead to chaotic emission. Due to the coexistence of two oscillations this scenario is called torus regime. Section 6.5 presents the intricate dynamics in detail.

\section{Self-stabilization of feedback phase}

Comparing pulsation islands in the MB regime for the PFL (Fig. 3.5) and the AFL (Fig. 4.7), the difference in their extension is obvious. In the PFL, beating pulsations are found only in a small range of feedback phase, whereas in the AFL the pulsations can be stable over a whole period.

A detailed investigation of the effects stabilizing the beating pulsations has been given by Brox et al. in [22]. The reason for the broad pulsation areas in the parameter plane of $I_{P}$ and $I_{A}$ is the interrelation of feedback strength and feedback phase, which is introduced by the carrier density in the amplifier. Changing $\phi_{P}$ also modifies the carrier density in the amplifier section. This gives an additional contribution $\Delta \phi=$ $-\alpha_{H} \Delta g_{A}\left(n_{A}\right) L_{A}$ to the effective feedback phase. When fixing the amplifier current and increasing $\phi_{P}$, the feedback strength $K$ falls within an MB-island due to a readjustment of the carrier density $n_{A}$ in the amplifier section. The shift of the total feedback phase varies less than $\phi_{P}$ by the same reason. Due to the dependency of pulsation frequency on the feedback strength $K$, a change of $\phi_{P}$ tunes the frequency. Once the amplifier reaches saturation, $K$ becomes independent from $I_{A}$ and the frequency has to be adjusted by the feedback phase (cf. Fig. 4.9).

The self-adjustment of the feedback parameters allows for the preparation of robust high-frequency pulsations over a wide range of injection currents. Additionally, the 
self-stabilization of MB pulsations makes them robust against internal and external perturbations.

\section{Importance of passive section}

Improving the PFL and solving the problem of controlling feedback strength, the integration of an additional amplifier section seems a logical step in the device development. However, changing $I_{A}$ already modifies both feedback parameters $K$ and $\phi$ due to the non-zero linewidth enhancement factor $\alpha_{H}$. Therefore, a device integrating only an amplifier section for feedback control (subsequently called DFB-A configuration) would be even more straightforward than the AFL.

Since the simulation results for the AFL showed very good agreement with the measurements, the DFB-A device concept was investigated by simulations. The compound feedback cavity was replaced by an active section of $600 \mu \mathrm{m}$ length, while other device parameters remained identical. This enables the direct comparison to the results obtained for the AFL. The DFB current is varied from 30 to $100 \mathrm{~mA}$ and the amplifier current from 0 to $100 \mathrm{~mA}$. Therefore, when both currents act orthogonally, all combinations of feedback strength and phase should be accessible.

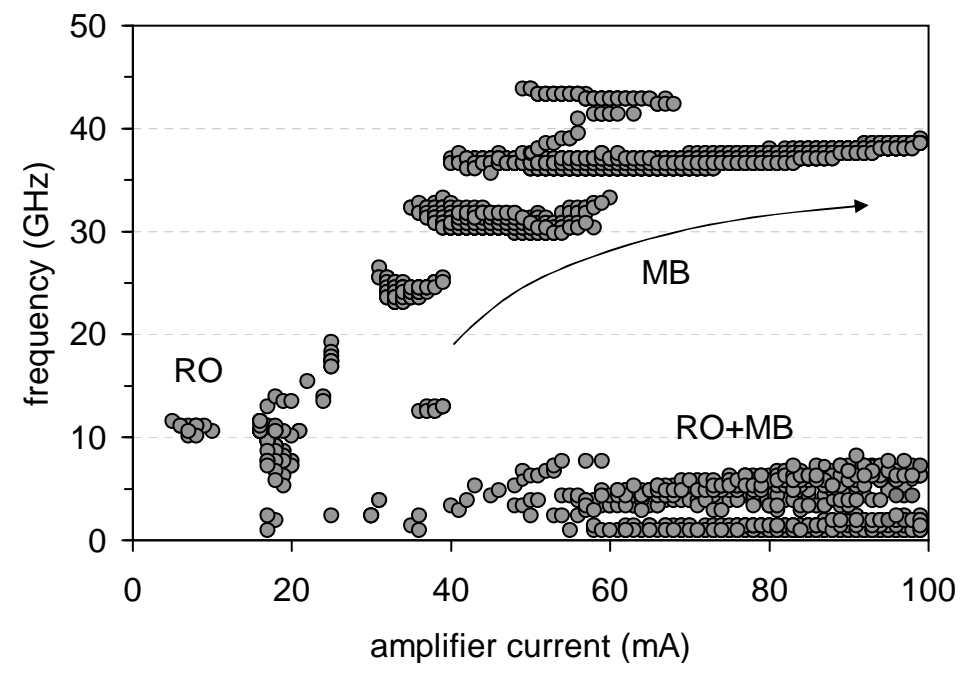

Figure 4.10: Control of pulsation frequencies with the amplifier current in a DFB-A configuration. Results for DFB currents from 30 to $100 \mathrm{~mA}$ are summarized. Frequency gaps occur due to dynamical interaction of carrier densities in the active sections.

Figure 4.10 summarizes the pulsation frequencies when changing the injection into both laser sections. Comparing the simulations for the DFB-A device with the according results for the AFL in Fig. 4.9, it is obvious that the same pulsation types occur. The upper frequency limit corresponds to the AFL ( $44 \mathrm{GHz}$ at $\left.I_{A}=50 \mathrm{~mA}\right)$. Operation points with higher amplifier currents are characterized by a RO/MB coexistence.

A striking difference to the AFL is, that some frequency areas are not addressable. The reason is the self-adjustment of feedback parameters. As soon as mode beating occurs, the lasing modes stabilize each other at the threshold density. Hence, an effective 
means for changing $\phi$ is lacking, because it is a slaved parameter of the carrier density $n_{A}$. To enable a continuous frequency tuning range, a separate control mechanism for the feedback phase is required. In consequence, the AFL represents the minimum setup for a DFB laser with active feedback which allows to address all possible dynamical states.

In summary, this Chapter has shown that the concept of the AFL enables devices with a separate control of feedback strength and phase. Due to the large range of accessible feedback strengths RO and MB pulsations can be prepared. For a detailed understanding of the dynamics it is necessary to know the bifurcations organizing the transitions between different states. The next Chapter summarizes theoretical results obtained for the AFL. They will be experimentally verified in the subsequent Chapters. 


\section{Chapter 5}

\section{Bifurcations of the AFL: theoretical predictions}

The first exploration of AFL behavior has shown that various dynamical phenomena occur. Since lasers with optical feedback are highly nonlinear systems, bifurcation theory is an appropriate tool for analyzing the dynamics. This Chapter presents a complete bifurcation diagram for the AFL [12]. It serves as a guideline for the further characterization. Additionally, the relevant bifurcation types are discussed.

\subsection{Bifurcation diagram}

Bifurcation theory is a powerful instrument for the investigation of nonlinear dynamical systems (for an overview the reader is referred to Refs. [40, 57]). It is routinely applied for the investigation of laser dynamics [55].

An interdisciplinary collaboration with mathematicians at the WIAS has been started, in order to obtain an overview on the bifurcations arising in the AFL. In the framework of this cooperation a complete bifurcation diagram has been calculated by J. Sieber [12] using path-following techniques. This method is based on detecting different bifurcation types. Subsequently, these bifurcations are followed in parameter space, giving the boundaries between regions of qualitatively different dynamics.

To make the traveling-wave description of the AFL tractable for the available pathfollowing tools $[28,35]$, an approach based on expanding the optical field in terms of hot cavity modes was used $[4,91]$. Restricting to RO and MB phenomena, which dominate the AFL dynamics, only two master modes are taken into account [80].

While yielding a less accurate description of the total mode spectrum, these simplifications reproduce the relations between the two closely spaced master modes sufficiently well and thus have only marginal influence on the dynamics of this subsystem [75]. For the bifurcation analysis LSHB is ignored $(n=\bar{n})$, as well as gain dispersion $(\bar{g}=0)$ and nonlinear gain suppression $(\varepsilon=0)$ in the active sections.

Figure 5.1 depicts in the $\left(\phi_{P}, I_{A}\right)$-parameter plane all bifurcations obtained for $\mathrm{cw}$ states and the physically most relevant bifurcations of self-pulsations. For consistency reasons, the DFB injection is fixed to $70 \mathrm{~mA}$. The amplifier current axis is limited to 


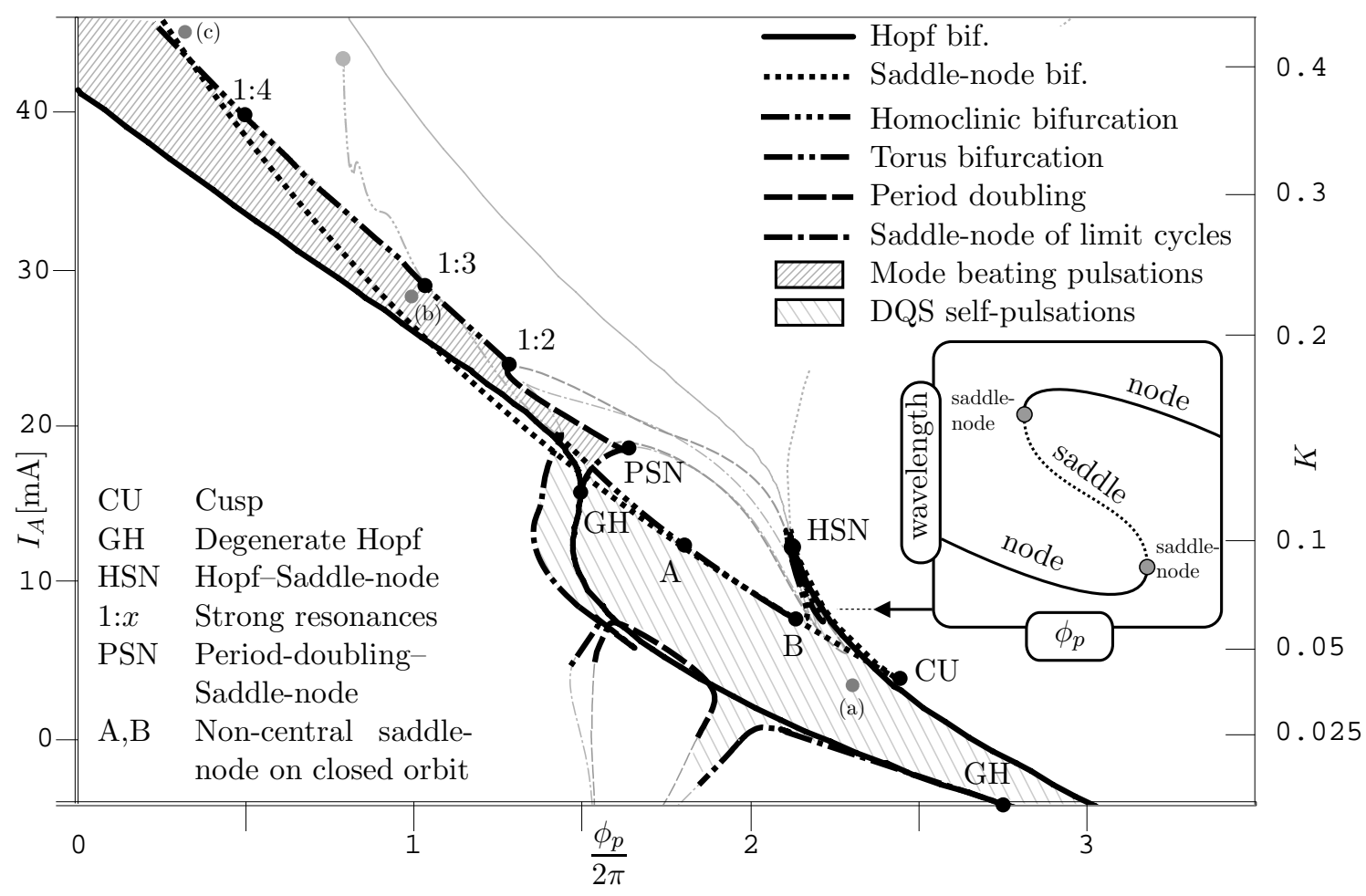

Figure 5.1: Bifurcation curves and islands of stable self-pulsations of the simplified twomode model in the $\phi_{P}$ vs $I_{A}$ parameter plane. Shaded areas indicate stable RO (DQS) and MB pulsations. The right hand scale is the feedback strength at the lower Hopf bifurcation. Parameters are identical to Appendix A.1, except $\bar{g}=0, \varepsilon=0, \alpha_{H}=-4$, and fixed $\alpha_{P}=$ $20 \mathrm{~cm}^{-1}$. Inset: wavelength of stationary state vs phase shift $\phi_{P}$ schematically illustrating the double-fold structure above the cusp. The dynamics in the points (a)-(c) is illustrated in Fig. 5.2 (bifurcation diagram: J. Sieber [12]).

the range where no more than two modes are dominant in the simulation of the full TWEs. Shaded areas represent the islands of different types of stable self-pulsations. The lower one corresponds to undamped single-mode ROs, MB self-pulsations occur in the upper island. The two islands are tilted and extend over several phase periods. All these features agree very well with the numerical and experimental findings. The extension of the islands is larger than in the full numerical solution (Fig. 4.8). This is mostly due to the disregard of gain saturation, by which the damping of the ROs is underestimated. Recurring islands at higher phase periods do not occur, as only one selected pair of modes is considered. Bifurcations are shown in gray where the mode approximation leaves the range of validity.

Based on the overview given in Fig. 5.1 the different bifurcations relevant for the device dynamics are now introduced. Consequences for the dynamics are discussed with respect to the AFL. 


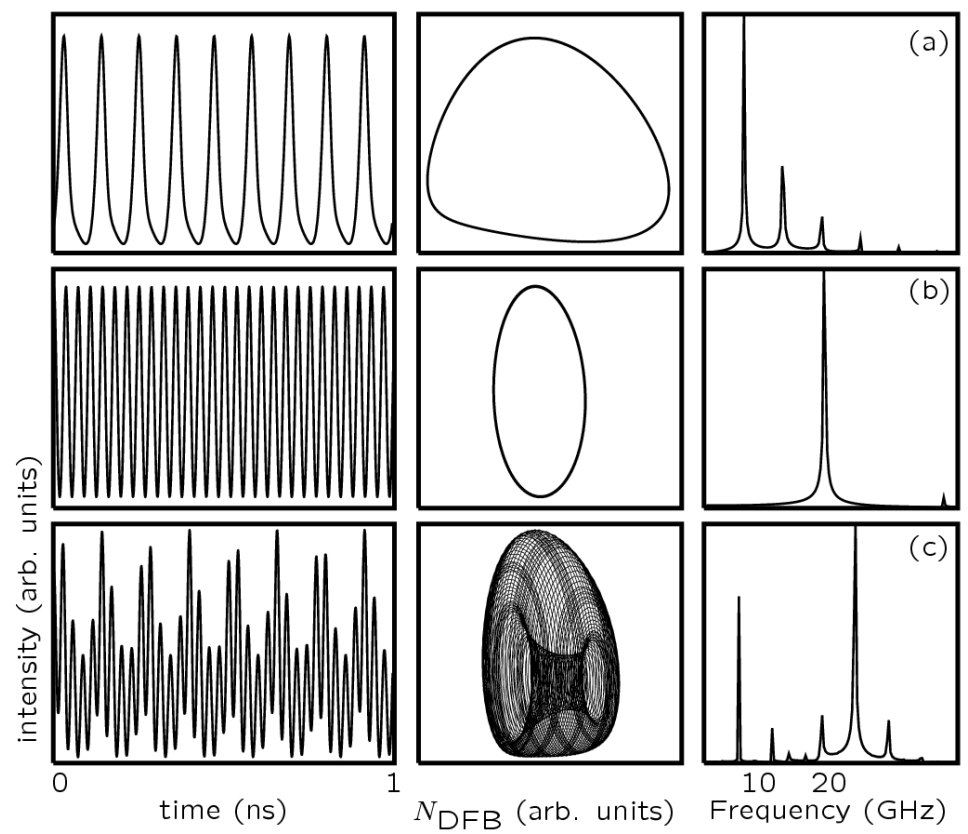

Figure 5.2: Dynamics in the points labeled (a)-(c) in Fig. 5.1. (a) Typical low frequency RO self-pulsation, (b) MB self-pulsation, and (c) beyond the torus bifurcation. Left column, output power vs time; middle column, projection of the phase space trajectory onto the plane of output power vs DFB carrier density, and right column, power spectrum.

\subsection{Folds of stationary states}

Equilibrium states in the AFL can undergo two types of bifurcations. Under the variation of a single parameter (co-dimension one) either Hopf or saddle-node (SN) bifurcations are observed.

SN bifurcations in the feedback setup correspond to the creation of additional modepairs [65]. The basic fold structure due to the coexistence of several compound-cavity modes has already been discussed in Section 3.1.2. In our investigation of the AFL the feedback range where a single or three stationary states coexist are of interest. A comparison of Fig. 3.3 with the bifurcation diagram in Fig. 5.1 shows a similar fold structure due to SN instabilities. The amplifier section gives an additional contribution to the feedback phase, therefore modifying the location of SN bifurcations. From the cusp (CU) point the SN curves (dotted lines) emanate, separating the regions with a different number of $\mathrm{cw}$ states. One branch of the SN curve traverses to HSN (Hopf saddle-node), the other to the points A and B. In between the SN branches three cw states coexist. Above the point HSN the SN curve is shown in gray since it not accessible in experiment. This is due to other bifurcations, which destabilize the stationary state already before reaching the right SN branch. An example for such an instability is the Hopf bifurcation discussed in the next Section. 


\subsection{Hopf bifurcations}

Hopf bifurcations are another co-dimension one bifurcation of stationary states. Here, a fixed point solution looses stability and a periodic orbit with small amplitude is born. Two different modifications of the Hopf bifurcation occur - the sub- and the supercritical type. In the supercritical case, a stable state bifurcates into an unstable equilibrium and an attracting limit cycle. When moving away from the bifurcation point the orbit expands. The subcritical case is characterized by an exchanged stability of equilibrium state and limit cycle, giving rise to a discontinuous change of the laser state. This scenario can be the boundary of a hysteresis loop. Both types of Hopf bifurcations are illustrated in Fig. 5.3.

a) supercritical Hopf

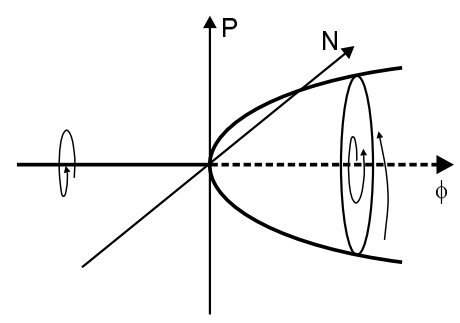

b) subcritical Hopf

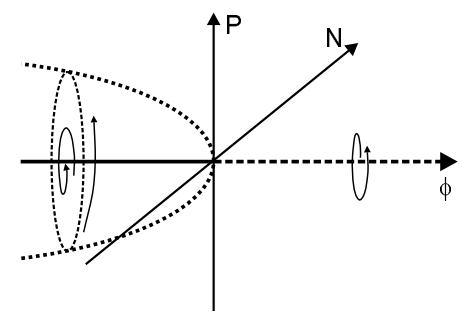

unstable

stable

Figure 5.3: Schematic illustration of Hopf bifurcations in phase space. Coordinates: optical power $P$, carrier density $N$, and bifurcation parameter $\phi$.

The bifurcation diagram Fig. 5.1 shows that the onset of undamped $\mathrm{RO}$ and $\mathrm{MB}$ pulsations at the left island border is given by Hopf bifurcations (solid lines). Below the CU point undamped ROs also end in a Hopf bifurcation (for increasing $\phi_{P}$ ).

At low feedback strengths the Hopf bifurcations induce undamped RO pulsations. Here, the damping of the relaxation oscillations becomes zero and periodic pulsations of the laser output occur. The dynamics is illustrated in the first row of Fig. 5.2. Pulses have a non-sinusoidal shape, leading to higher harmonics of the fundamental frequency and a deformed trajectory in phase space. The RO self-pulsation is stable for the supercritical Hopf bifurcation above the upper Generalized Hopf (GH, also called Degenerate Hopf) point and below HSN. Between the two GH points the Hopf bifurcation is subcritical. Section 5.4.2 will illustrate the scenario in this area.

MB pulsations occur on the Hopf curve at higher amplifier current. A second mode reaches threshold here, whereas the relaxation oscillations remain strongly damped. Periodic solutions appear as bridges between stable and unstable modes [71]. The middle row of Fig. 5.2 illustrates the MB dynamics. Within the MB island, the power is distributed between the two modes such that both keep at threshold.

Once the laser is in a pulsating RO or MB state, further bifurcations of periodic orbits can occur. These will be discussed in the following Sections. 


\subsection{Bifurcations of limit cycles}

\subsubsection{Homoclinic bifurcation}

After the onset of pulsations at the left island border different bifurcations can occur, either causing a pulsation break-down or leading the system into more complex dynamics. A possible scenario is the collision of a limit cycle with a saddle-point, giving rise to a homoclinic bifurcation. The bifurcation analysis for the AFL shows that the right border of the $\mathrm{RO}$ island above point $\mathrm{B}$ is given by a homoclinic bifurcation (triple-dot-dashed curve in Fig. 5.1). The situation is illustrated in Fig. 5.4.

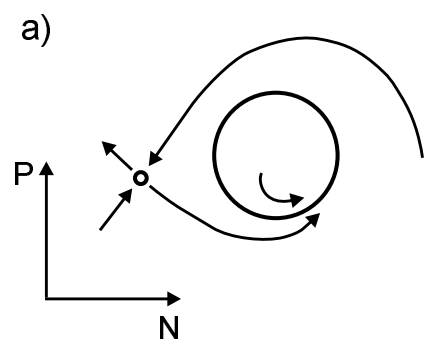

b)

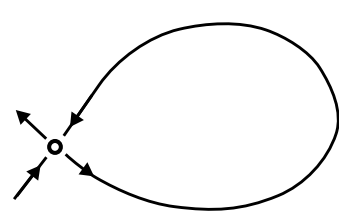

Figure 5.4: Illustration of the phase space scenario for a homoclinic bifurcation. (a) The periodic orbit comes close to a saddle; (b) the collision of periodic orbit and saddle results in a homoclinic connection of stable and unstable manifold.

When approaching the homoclinic, the limit cycle of the RO pulsations comes close to a saddle. Due to the saddle, the trajectory is strongly slowed down. Consequently, the frequency of the self-pulsation becomes smaller. As soon as the limit cycle and the saddle collide, the RO pulsations disappear and the laser switches to a stable cw state. The switching between the periodic orbit and the stable state occurs without hysteresis if the connection in the phase space is actually towards a saddle-node, i.e. if the curve of homoclinic bifurcations coincides with the curve of SNs between the points $\mathrm{A}$ and B in Fig. 5.1.

\subsubsection{Saddle-node of limit cycles}

The other bifurcations bordering the RO island were not directly observed in experiment. However, they influence the device dynamics with respect to hysteresis and the impact of noise. For completeness they are briefly discussed here.

Saddle-nodes of limit cycles (SNOLC, single-dot-dashed line in the bifurcation diagram) appear at the left boundary of the DQS island located close to the Hopf bifurcation. Figure 5.5(a) illustrates the phase space scenario for a SNOLC bifurcation. At the point 1 an attracting and a saddle-type periodic orbit are born. Both periodic orbits move away from each other, until at point 2 the unstable attractor disappears and only the stable orbit remains. Depending on the direction in which the SNOLC is approached, different dynamics can be expected. From the left, a subcritical Hopf 
a)

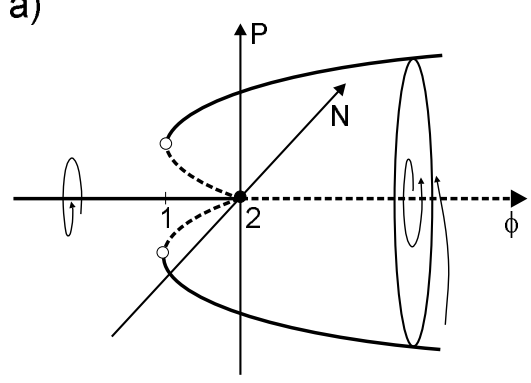

b)

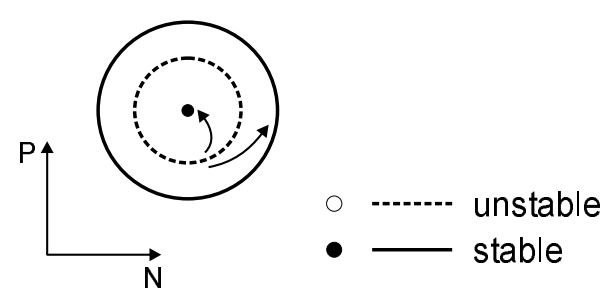

Figure 5.5: (a) Illustration of saddle-node of limit cycle bifurcation. (b) Coexisting stable and unstable limit cycles between the points 1 and 2 .

bifurcation is observed, i.e. a sudden jump to a limit cycle. In the reverse direction, a jump from the stable limit cycle to another regime can be expected.

In the bifurcation diagram, the SNOLC width shrinks to zero at the upper GH point, changing the Hopf bifurcation from subcritical to supercritical for increasing $\phi_{P}$. There is not much hysteresis with respect to a parameter variation even at the subcritical Hopf bifurcation boundary of the DQS island between the two GH points. The reason is the proximity of the Hopf curve to the curves of PD and SNOLC.

\subsubsection{Period doubling}

Another bifurcation of limit cycles is period doubling (PD, dashed line). In general, a PD bifurcation creates a new limit cycle with twice the old period (compare Fig. 5.6(a)). PDs are predicted for the undamped ROs in the area enclosed by the dashed line. For the MB pulsations, PD appears at the right island border. Cascades of PDs are a possible route leading the AFL dynamics into chaos.

\subsubsection{Torus bifurcation}

Torus bifurcations arise for laser states with MB pulsations, when a limit cycle undergoes a second Hopf instability. This results in a motion located on a torus in phase space. Figure 5.6(b) schematically depicts the scenario of a torus bifurcation. Similar to the PD, torus bifurcations can be sub- or supercritical. For the AFL, the torus onset is supercritical along most parts of the MB island, i.e. a stable torus is born. This has been validated by numerical tests. Both, the fast $\mathrm{MB}$ and the ROs are undamped here (cf. lower row in Fig. 5.2). A stable torus bifurcation manifests itself by a smooth rise of secondary peaks in the power spectrum below and above the main frequency peak. Close to the strong resonances $(1: 2,1: 3,1: 4)$, the secondary peaks are of low rational order and should be particularly pronounced.

Quasiperiodic orbits occur for non-rational frequencies of MB and RO. The periodicity of a torus can be analyzed using Poincaré maps. They are obtained as sections of the trajectory in phase space. For a locked state, discrete points appear; a quasiperiodic 
a)

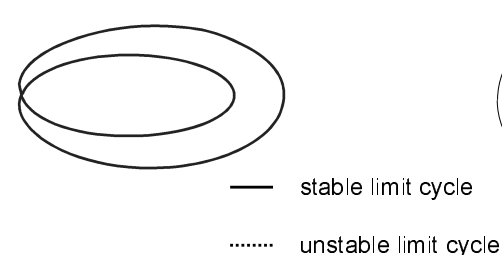

b)

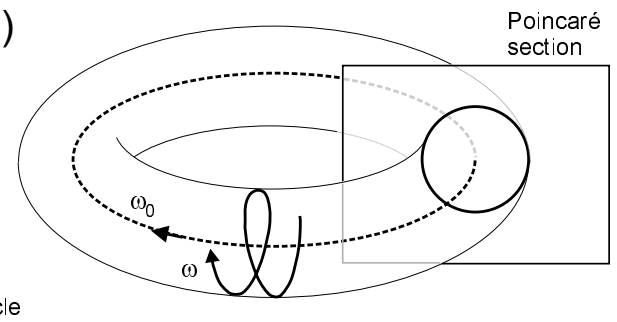

Figure 5.6: Phase space illustration of dynamics after (a) PD bifurcation, (b) torus bifurcation. The motion on the torus is characterized by different frequencies $\omega$ and $\omega_{0}$. A Poincaré section for a quasiperiodic torus is illustrated.

orbit gives a closed curve in the Poincaré section.

The presented bifurcation analysis has not been used to investigate the behavior in the torus region. Here, several interesting phenomena can occur. Detailed experiments will be presented in following Chapters. 


\section{Chapter 6}

\section{Experimental verification of bifurcations}

So far, experiments and simulations have shown good agreement with respect to the basic dynamical phenomena in the AFL. Based on the bifurcation overview, this Chapter gives a detailed experimental analysis of dynamics. Bifurcations of $\mathrm{cw}$ and pulsating states are investigated.

\subsection{Detailed overview on device dynamics}

The overview extracted from the main peaks of the power spectrum (Fig. 4.7) verified that the global experimental behavior is conform with simulations as well as with the results from the bifurcation analysis. An in-depth comparison, however, requires a closer look to the dynamics.

Figure 6.1 shows optical spectra and $\mathrm{rf}$ power spectra for parameter combinations $I_{D F B}=70 \mathrm{~mA}, I_{P}=8 \mathrm{~mA}$, and $I_{A}=[0-100] \mathrm{mA}$ (left and right columns). A representative overview on the characteristic phenomena in the AFL is obtained by a variation of $I_{A}$ only. The optical spectra are shown close to the left stop-band mode, which remains dominant in the investigated range $(>20 \mathrm{~dB}$ suppression of modes not involved in dynamics). An additional measurement for decreasing amplifier current illustrates hysteresis effects (middle column). The laser output is measured from the amplifier facet, explaining the increase of optical power with $I_{A} \cdot{ }^{1}$

$\mathrm{Rf}$ and optical spectra allow for the identification of characteristic dynamical states. At low feedback cw emission occurs (I), changing into undamped ROs for higher feedback (II). Increasing $I_{A}$, the transition region from RO to MB pulsations (III) is crossed. In the $\mathrm{MB}$ regime (IV, V, VI) pure $\mathrm{MB}$ or the interaction with the undamped ROs (torus scenario) arises. Starting from this overview, the predictions from the bifurcation analysis are now verified.

\footnotetext{
${ }^{1}$ Spectra measured at the DFB laser facet show the same features, however, individual modes may have differing amplitudes due to spectral filtering effects of the DFB section.
} 


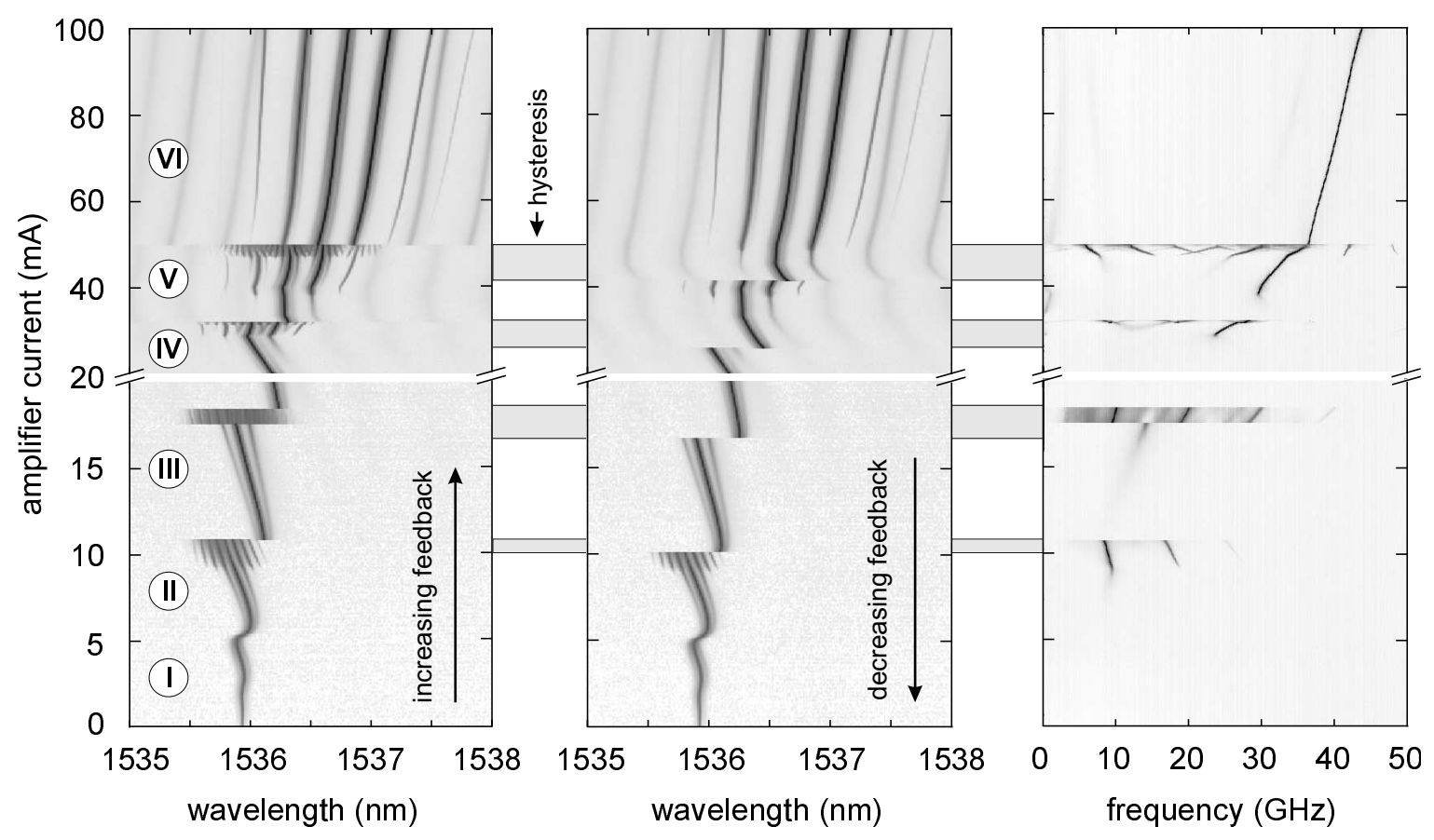

Figure 6.1: Overview on dynamical regimes in AFL. Phase current fixed at $8 \mathrm{~mA}, I_{A}$ varied. The left two diagrams show intensity plots of optical spectra, recorded for increasing (left) and decreasing injection (middle), hysteresis regions are indicated. The right diagram displays power spectra measured for increasing feedback. Intensities in the diagrams are encoded with logarithmic gray scale.

\subsection{Hysteresis}

The overview measurement in Fig. 6.1 reveals large areas of hysteresis in the device dynamics. Hysteretic behavior is induced, when several stable states coexist at a parameter combination. It has already been discussed in the previous Chapter, that a main source for hysteresis in the AFL is the fold structure due to the saddle-node (SN) bifurcations (see Fig. 5.1). The basic fold structure is modified by additional bifurcations, which destabilize the stationary states. As a result, the right branch of the SN curves can not be detected in experiment and the hysteresis behavior of the AFL is governed by the left SN branch and the respective bifurcation bordering the pulsation islands.

In order to identify the borders of hysteresis, it is required to measure the device output in every operation point for the different directions of feedback parameter variation, similar as it was presented in Fig. 6.1. The measurement time, however, limits the application of this method to the most interesting points. ${ }^{2}$ A rather time consuming part is the measurement of the optical spectra. Therefore, a fast characterization

\footnotetext{
${ }^{2} \mathrm{~A}$ few numbers should illustrate this. The spectra in Fig. 6.1 were recorded with steps of $0.02 \mathrm{~mA}$ in $I_{A}$, requiring two hours of measurement time. This makes an equally dense variation of all other parameters not feasible.
} 

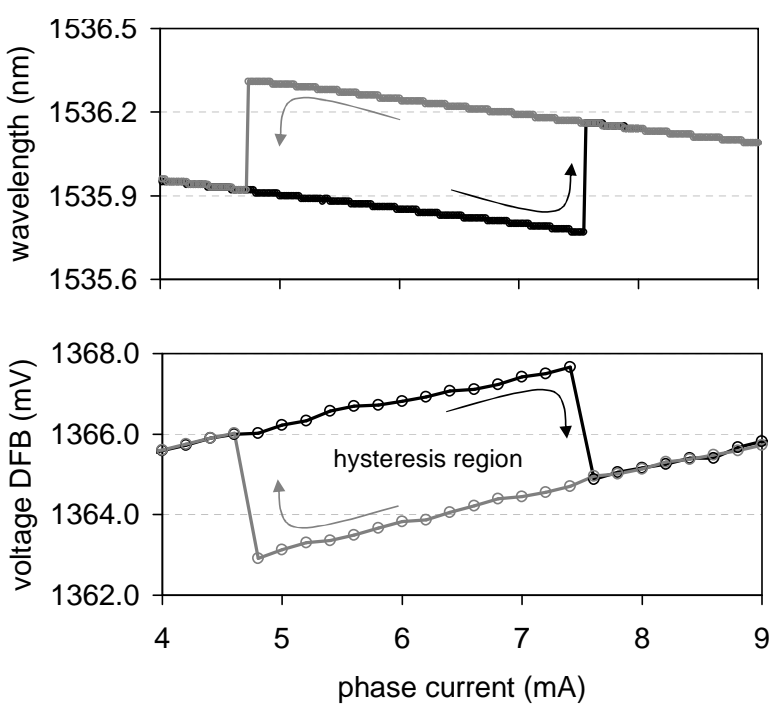

Figure 6.2: Hysteresis at $I_{A}=21 \mathrm{~mA}$ for $I_{P}$ variation. The upper curves show the evolution of peak wavelength, the lower diagram indicates the junction voltage measured at the DFB section $\left(I_{D F B}=70 \mathrm{~mA}\right)$.

technique has to detect the mode jumps by different means. One can take advantage of the fact that a mode jump is associated with a redistribution of the carrier densities within the device. The changes in the carrier density modify the Fermi levels in the pn-junction of the active sections. This affects the junction voltage, a measurable quantity. Figure 6.2 shows a comparison of both techniques. Discontinuities of the junction voltage for increasing and decreasing $I_{P}$ coincide with the jumps in lasing wavelength. Larger values of the junction voltage correspond to a higher carrier density, explaining the observed decrease in lasing wavelength due to the change in refractive index. Parameter values, where the voltages or wavelengths are different for both measurement directions mark the hysteresis region. Hence, the measurement of junction voltage is a reasonable method for detecting mode jumps.

The recording of junction voltage also allows to verify results from simulations regarding the redistribution of carrier densities in different operation points. Such a comparison requires a relation between discontinuities in the carrier density $\Delta N$ and the voltage $\Delta V$. Under the assumption that the effective density of states in the conduction band $N_{c}$ is much smaller than $N_{v}$ in the valence band [48], the relation

$$
\Delta V=\frac{k_{B} T}{e}\left(\frac{2}{n_{t h}}+\frac{1}{\sqrt{8} N_{c}}\right) \Delta N
$$

can be deduced (Joyce-Dixon approximation). Here, $k_{B}$ denotes the Boltzmann constant, $T=300 \mathrm{~K}$ the device temperature, $e$ the electron charge, and $n_{t h}$ the carrier density at threshold. Using the parameters from the device simulation $\left(n_{t h}=\right.$ $1.6 \times 10^{24} \mathrm{~m}^{-3}, N_{c}=5.3 \times 10^{23} \mathrm{~m}^{-3}$, estimated from the effective mass [76]), the voltage change of $2.7 \mathrm{mV}$ corresponds to a carrier density variation of $\Delta N=0.055 \times 10^{24} \mathrm{~m}^{-3}$. 


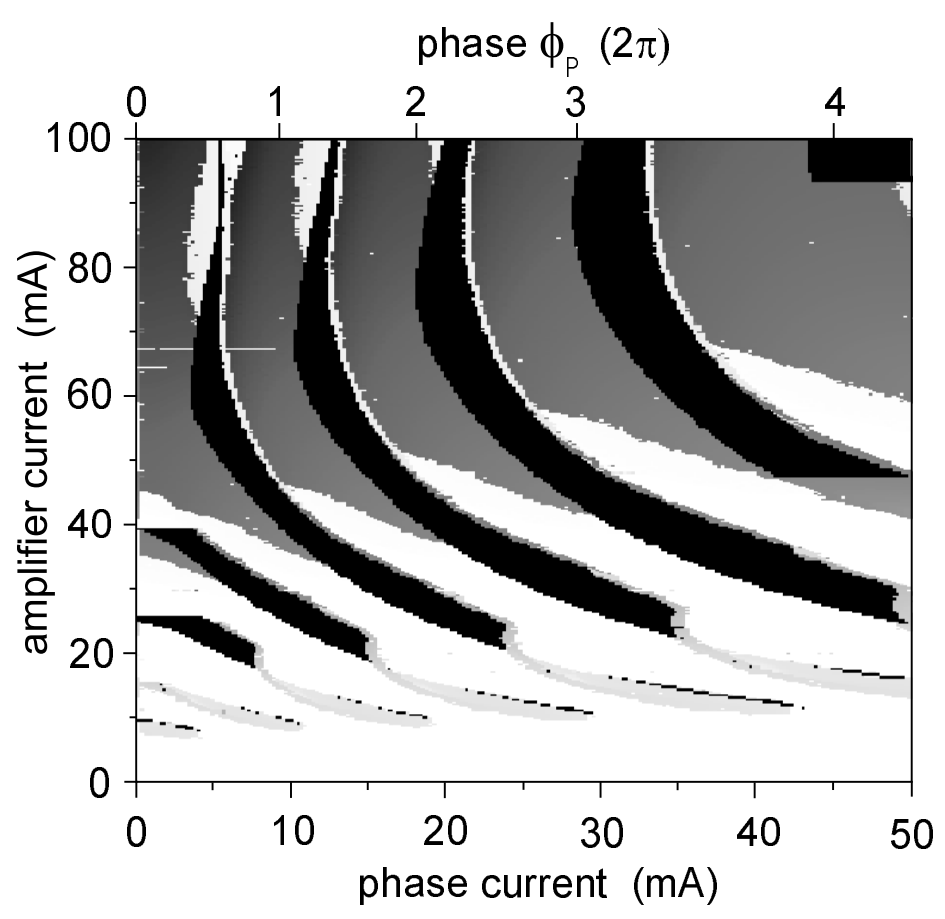

Figure 6.3: Hysteresis regions determined from bidirectional measurements of the DFB junction voltage (black areas). Gray areas show the pulsation islands for comparison.

In simulation, a carrier density jump of $\Delta N=0.12 \times 10^{24} \mathrm{~m}^{-3}$ was obtained for an equivalent $\mathrm{MB}$ pulsation area (16 mA, left island in Fig. 4.8). This is a reasonable agreement, taking into account the uncertainty in the experimental values of $n_{t h}$ and $N_{c}$.

The global hysteresis structure is obtained by repeating the measurements of junction voltage in forward and backward direction for the whole parameter range [76]. Figure 6.3 summarizes the results obtained with $I_{P}$ as primary bifurcation parameter. Areas of hysteresis are indicated in black. The hysteresis regions are superposed to the pulsation island overview from Fig. 4.7 for illustration.

Different scenarios lead to discontinuous transitions of the laser state and enable the measurement by detecting junction voltage jumps. The jumps occur, when several stable states coexist. Hence, the cusp (CU) point, indicating the lower border of coexisting laser states, can be determined from the onset of hysteresis. For the investigated device the $\mathrm{CU}$ point is located at $I_{A}=8 \mathrm{~mA}$ (first island), i.e. almost at the lower island border opposed to the bifurcation analysis. The difference can be explained by the disregard of nonlinear gain saturation, which leads to an overestimation of the RO pulsation area. Additionally, the value of $\alpha_{H}=-5$ in experiment moves the cusp point to lower $I_{A}$, as the minimum feedback strength required for the coexistence of three cw states scales with $K_{\min } \propto 1 / \sqrt{1+\alpha_{H}^{2}}$ (cf. Section 3.1).

Approaching the hysteresis area from the left, the AFL is in a pulsating state. This pulsating state becomes unstable already before the right SN branch of the fold 
structure, causing the jump to the coexisting stable mode. In the reverse direction, the left SN induces the change of the laser state. Here, the laser typically jumps into a pulsation. A verification that the jumps in reverse direction are actually induced by a SN bifurcation is given by the characteristic variation of the lasing wavelength close to the mode jumps (compare Fig. 6.1 middle column). Reason for the wavelength variation is the change of threshold gain close to the bifurcation point (cf. Fig. 3.2(b)).

Since the CU point is located at the lower border of the pulsation island, ROs show a small hysteresis $(<1 \mathrm{~mA})$ at the right island border. Towards the transition to MB pulsations hysteresis disappears due to the coincidence of homoclinic and saddle-node bifurcation. Between $20 \mathrm{~mA}$ and $60 \mathrm{~mA}$ the hysteresis width strongly increases. The width of the fold can be larger than the area of pulsations. As a result, MB pulsations can only be addressed from the left. In the reverse direction, jumps between stationary cw emission can be observed. Beyond $60 \mathrm{~mA}$, the width of the hysteresis region reduces.

The experimentally determined hysteresis structure shows a good agreement to the behavior predicted by the bifurcation diagram. It serves as a map for further investigations, indicating regions where the direction of feedback parameter variation has to be taken into account. This is especially important for the MB pulsations, where the whole island can disappear due to the fold structure (cf. Fig. 6.1, middle row). Artifacts in the fold determination in Fig. 6.3 (lower left and upper right corner) appear, when measurements do not leave the hysteresis region. This effect has to be regarded in the determination of folds, when changing $I_{A}$ as bifurcation parameter. At low injection into the amplifier section, measurements for $I_{A}$ as parameter coincide with the results obtained for $I_{P}$. However, the vertical alignment of pulsation islands for high injection disables the detection of the right fold border, i.e. the same mode-pair is observed independent of the amplifier injection. Consequently, the fold structure obtained by varying $I_{A}$ is not valid in this range.

Investigations of the hysteresis structure for devices with small modifications have proven that the fold width depends sensitively on device parameters. A laser with a phase section length of $550 \mu \mathrm{m}$, but otherwise identical parameters, has shown strongly reduced hysteresis for MB pulsations. For low amplifier currents the coincidence of the left SN branch with the border of the island has been observed. This results in a vanishing hysteresis. New dynamics is expected here, since internal or external noise can switch between different states.

The considerations in this Section only accounted for the right border of the pulsation islands. Of course, hysteresis may also occur at the left border. Later investigations will reveal that here only smooth transitions of the laser state occur, which can not be detected by a measurement of Fermi-voltage discontinuities. However, already the overview measurement in Fig. 6.1 illustrates that neither the onset of MB nor RO pulsations shows large hysteresis. 


\subsection{Hopf bifurcations}

Hopf bifurcations change the AFL dynamics from cw to pulsating emission. Depending on the feedback strength, either RO or MB pulsations are observed. Both pulsation types are now characterized.

\section{Undamped RO pulsations}

In region II of Fig. 6.1 undamped ROs are observed. The pulsations occur for appropriate combinations of feedback phase and strength. Figure 6.4 and Fig. 6.5 depict representative optical and rf spectra. The power spectrum with a frequency of $9 \mathrm{GHz}$ and a distinct second harmonic at $18 \mathrm{GHz}$ compares well with the results obtained in the numerical investigations (Fig. 5.2). The optical spectrum shows a splitting of the emission line into several subcomponents, the spacing corresponds to the $9 \mathrm{GHz}$ pulsation frequency. Frequency and spectral characteristics are typical for RO-type self-pulsations.

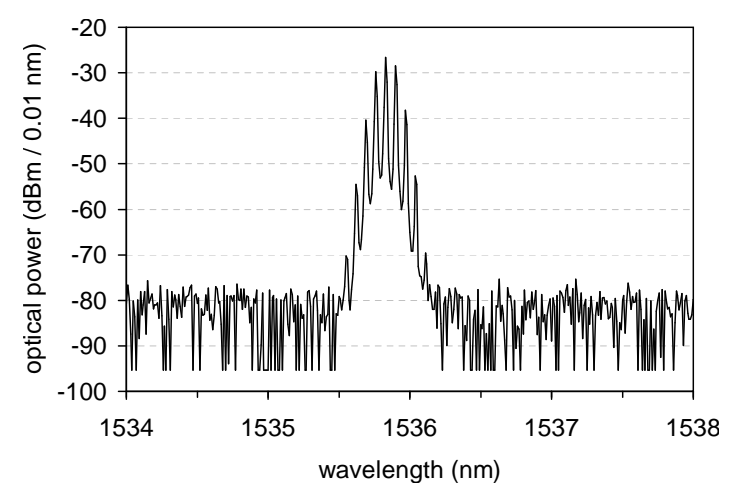

Figure 6.4: Optical spectrum of undamped ROs. The substructure of the pulsating mode corresponds to the oscillation frequency of $9 \mathrm{GHz} . \quad\left(I_{D F B}=70 \mathrm{~mA}\right.$, $I_{P}=8 \mathrm{~mA}$ and $\left.I_{A}=9.5 \mathrm{~mA}\right)$.

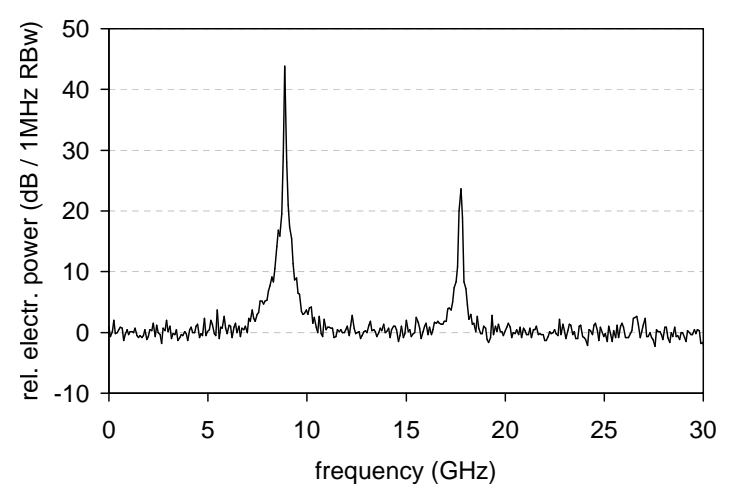

Figure 6.5: Power spectrum of undamped ROs. The linewidth is $10 \mathrm{MHz}$. A distinct second harmonic indicates the nonsinusoidal pulse shape.

From the onset behavior of the pulsation the type of Hopf bifurcation inducing the ROs can be determined. A smooth increase of oscillation amplitude without hysteresis indicates a supercritical Hopf bifurcation, whereas a jump is characteristic for the subcritical type. From the bifurcation analysis for the AFL the lower border of the $\mathrm{RO}$ island is expected to be subcritical. In order to verify this prediction, the amplifier current was tuned across the pulsation region. Figure 6.6 shows the evolution of height and position of the primary peak in the power spectrum.

The steep but continuous increase of the peak height at the lower island border $\left(I_{A}=9 \mathrm{~mA}\right)$ indicates a supercritical Hopf bifurcation causing the ROs. Together with the exponential increase of pulsation intensity the linewidth of the power spectrum reduces, indicating that the damping of the oscillations is decreased. Within the 


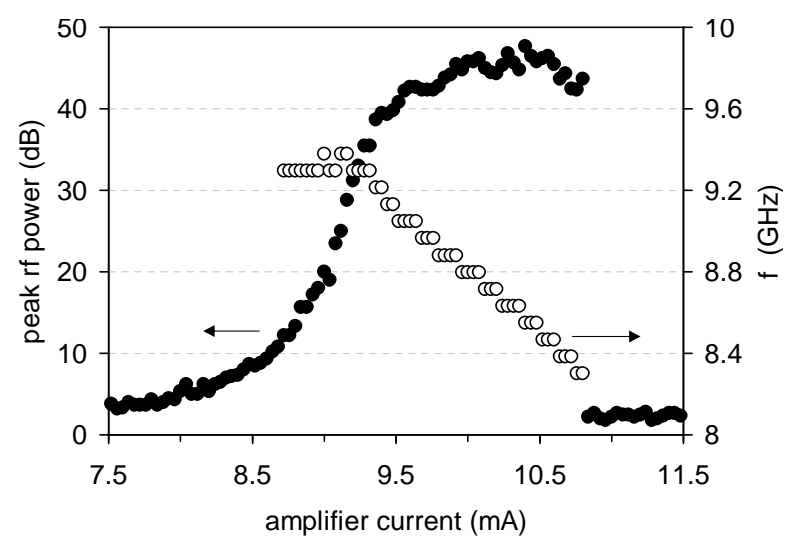

Figure 6.6: Dependency of RO rf peak power and frequency from amplifier current.

pulsation island the frequency changes continuously. After a slight increase during the undamping, a distinct slowing down is observed, which is accompanied by a further increase of the peak height.

It has been checked that below the cusp point both island borders are of Hopf type. The smooth onset and decay of oscillations indicates supercritical bifurcations.

\section{MB pulsations}

Mode beating is the second characteristic pulsation type induced by a Hopf bifurcation. Representative for the MB behavior, area $\mathrm{V}$ in Fig. 6.1 will be discussed. A magnified section of the power spectra is shown in Fig. 6.7. All considerations in this Section will focus on the dynamics below $I_{A}=46 \mathrm{~mA}$. Phenomena observed towards the island border will be treated in the following Sections. In the parameter range of interest, the power spectrum consists of a single spectral line close to $30 \mathrm{GHz}$. This

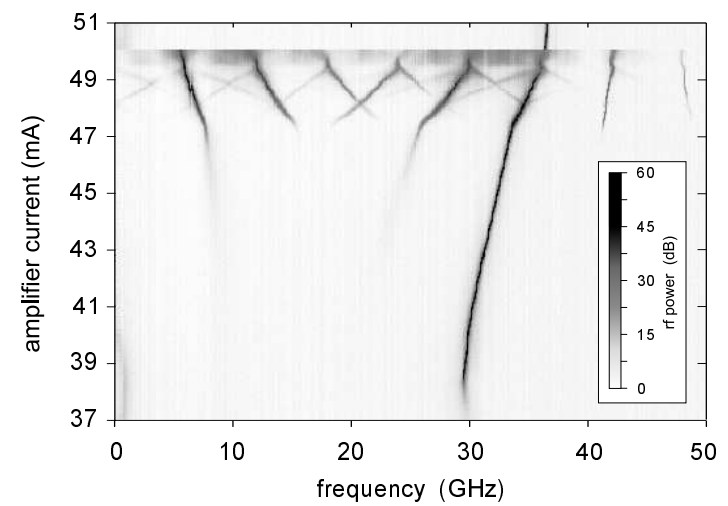

Figure 6.7: Characteristic power spectra for amplifier currents in the range from $I_{A}=$ $37 \mathrm{~mA}$ to $51 \mathrm{~mA}$. The gray scale encodes the spectral intensity. Data taken from Fig. 6.1.

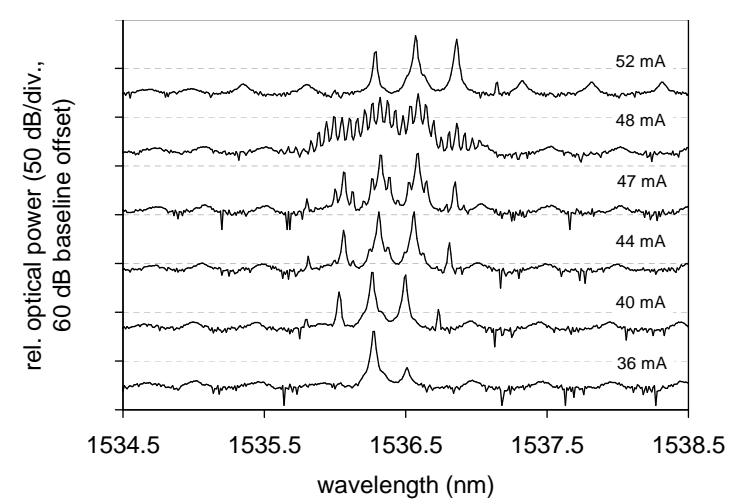

Figure 6.8: Optical spectra at selected points in Fig. 6.7. Above $I_{A}=47 \mathrm{~mA}$ complex spectra are the result of an interaction between RO and MB dynamics. 
is in good agreement with the theoretical prediction of stable MB pulsations. Possible higher harmonics are beyond the bandwidth of the spectrum analyzer. Equivalent measurements for lower MB frequencies show that only strongly suppressed second harmonics occur (suppression $>40 \mathrm{~dB}$ ). In this feedback range the beating frequency changes only moderately with the amplifier current. This observation is consistent with the decoupling of feedback strength $K$ from the amplifier current within the MB islands (see Fig. 4.8).

Typical optical spectra for MB pulsations are shown in Fig. 6.8. In case of a pure beating pulsation $\left(I_{A}=40 \mathrm{~mA}\right)$ two central peaks of comparable height are accompanied by one or more pairs of satellites. The central peaks represent the coexisting pair of optical modes. Their separation of $\sim 0.25 \mathrm{~nm}$ corresponds to the beating frequency of $30 \mathrm{GHz}$ and is considerably reduced with respect to the spacing of the ECMs. The satellites, separated from the main peaks by the same offset, are due to non-degenerate four-wave mixing of the two coexisting modes. The presence of such intense mixing products signifies a good spatial overlap of the two modes within the device and high phase stability of the MB pulsations. In accordance with a supercritical Hopf bifurcation the onset of MB pulsations is a continuous transition, smoothed by the presence of noise. The optical spectrum at $36 \mathrm{~mA}$ already shows the second mode being close to threshold. It is only weakly damped here and can collect a measurable amount of spontaneous emission. With increasing amplifier current, this peak rises quickly and approaches the height of the first one at $I_{A}=38 \mathrm{~mA}$. The peak rf power of $\mathrm{MB}$ pulsations plotted in Fig. 6.9 summarizes the onset behavior.

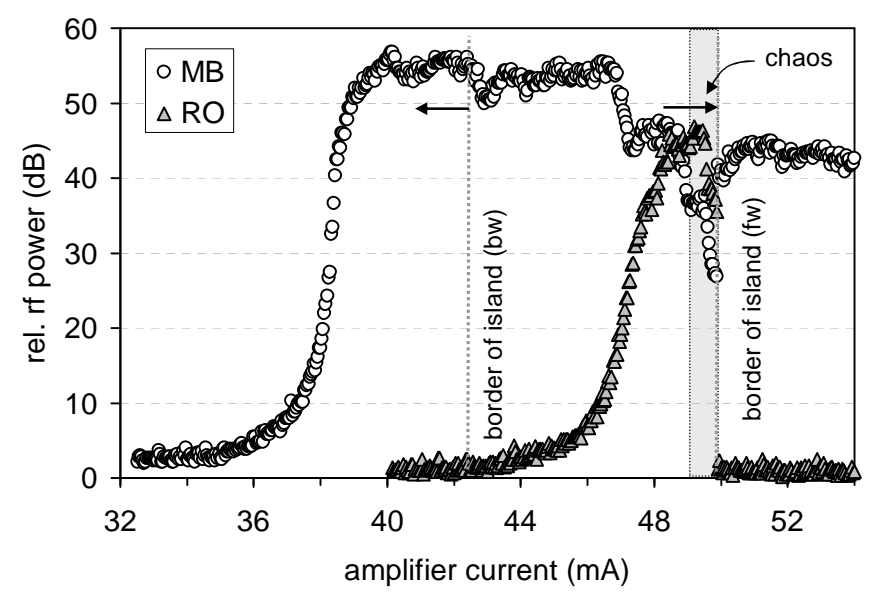

Figure 6.9: Onset behavior of MB pulsations and the transition to a torus scenario. Vertical lines mark island borders for the given direction of parameter variation (arrows). Towards the island border, chaotic emission is observed and low frequency spectral components become dominant. 


\subsection{Homoclinic bifurcation}

Another scenario bordering the pulsation island is the homoclinic bifurcation. The investigation of undamped ROs in Fig. 6.6 has shown an abrupt end of the pulsations at the upper island border. Such behavior is consistent with a homoclinic bifurcation above point A in Fig. 5.1. Due to the exponentially small region of the homoclinic, only a moderate slow down of the pulsation frequency $f$ towards this bifurcation occurs. Fluctuations, unavoidable in experiment, prevent a sufficiently smooth approach to the homoclinic and the observation of $f \rightarrow 0$. The investigation of the fold structure demonstrated that this border shows no hysteresis. This points at the coincidence of homoclinic and saddle-node bifurcation, as predicted by the bifurcation analysis. Approaching the RO island from the right between the points $\mathrm{A}$ and $\mathrm{B}$, also the onset of pulsations can occur due to the homoclinic bifurcation.

A direct evidence for the homoclinic can be given by the dynamics close to the bifurcation point. For the comparable experimental situation in a PFL, the homoclinic scenario has been verified with the demonstration of excitability in Ref. [96].

\subsection{Torus bifurcation}

The border of the MB island was investigated with respect to additional pulsations by determining the parameter regions, where a low frequency component occurs. For the measurements, the DFB injection was fixed to $70 \mathrm{~mA}$. The results are summarized in Fig. 6.10, for clarity only a single island is shown. Black areas indicate regions where at least one low frequency component in the power spectrum exceeds the noise floor by more than $10 \mathrm{~dB}$; gray areas show the MB island for comparison.

Along the border of the MB island either PD or torus pulsations are found. At low feedback strengths a large PD region is observed, which spreads over a large fraction of the island. Starting from the PD area, a narrow torus channel is emerging. The channel widens for higher feedback strengths and the MB frequency increases from 25 to $40 \mathrm{GHz}$. At the same time, a slowdown of the ROs to $6 \mathrm{GHz}$ is observed. Thus, the frequency ratio of $\mathrm{RO}$ and $\mathrm{MB}$ pulsations can be adjusted in a wide range. Both observations are in full agreement to the bifurcation analysis of the AFL.

The strong dependency of RO frequency on the feedback strength is a result of the feedback induced mode structure discussed in Section 4.3. Strong feedback makes the dispersion of mode damping close to the beating modes less distinct, i.e. modifications of the lasing wavelength induce a smaller variation of the gain. According to Eq. (3.11), this gives a lower enhancement of the RO frequency. Since the dispersion of mode damping flattens with feedback strength, an increase of MB frequency coincides with slower ROs.

The border of the MB scenario given in Fig. 6.7 serves as a good illustration of the characteristic torus behavior. A low frequency component emerges at $I_{A}=46 \mathrm{~mA}$ and coexists with the MB oscillator. The relaxation oscillation has noticeable higher harmonics and causes mirror satellites of the MB line, indicating a nonlinear interac- 


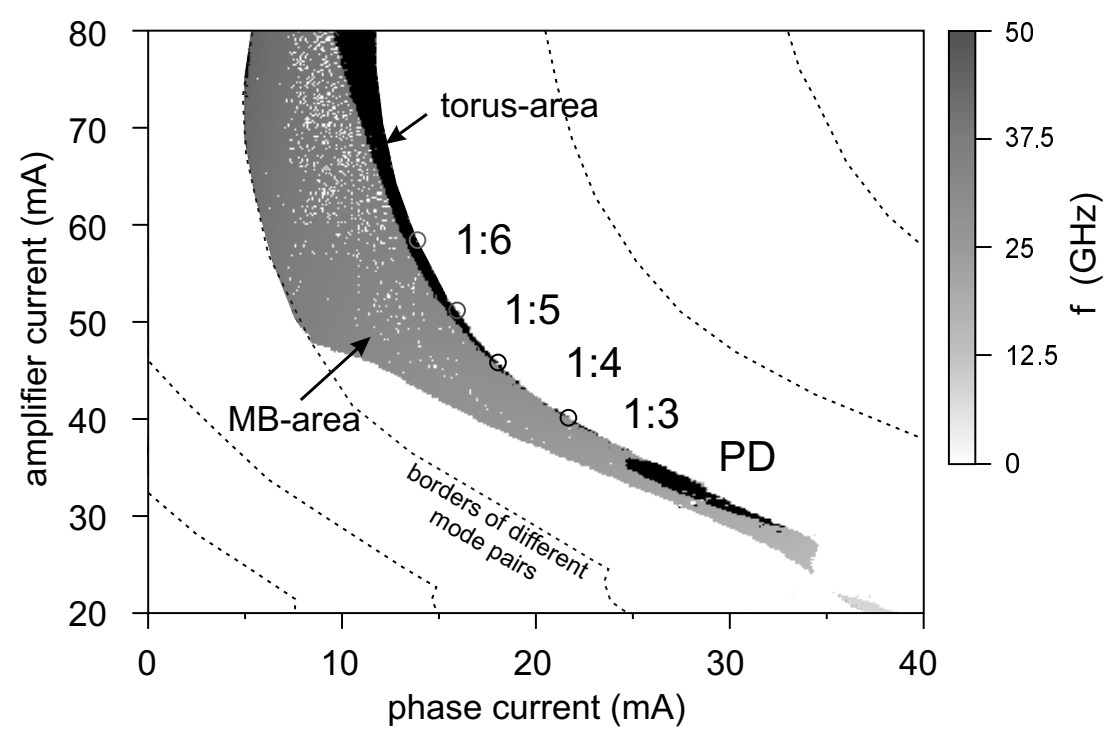

Figure 6.10: The black areas give locations of PD and torus pulsations. Results are shown for a single mode-pair. Positions of integer frequency ratios of the oscillators are denoted. Hatched lines are borders between adjacent mode-pairs.

tion between both oscillations. These phenomena are characteristic attributes of the torus bifurcation. The spectral intensities during the torus onset are shown in Fig. 6.9. A smooth increase of the RO component confirms the supercritical character. Weak shoulders of the main optical modes, already present in the spectra at $I_{A}=44 \mathrm{~mA}$ (Fig. 6.8), are precursors of this bifurcation. The shoulders become increasingly prominent and dissolve in individual sublines of the optical spectrum in the torus region. Due to the simultaneous change of both pulsation frequencies with the operation currents, different frequency ratios are obtained. This can give rise to internal synchronization phenomena, as observed just above $I_{A}=49 \mathrm{~mA}$, where the two oscillators synchronize each other at a 1:5 ratio within a $0.3 \mathrm{~mA}$ current range. 


\section{Chapter 7}

\section{The torus regime}

This Chapter characterizes the AFL behavior beyond the torus bifurcation. Resonances of $\mathrm{MB}$ and $\mathrm{RO}$ oscillations are investigated. Numerical simulations supplement the experiments by the demonstration of a torus break-up scenario. Additionally, a quasiperiodic route to chaos ending in a boundary crisis is shown.

\subsection{Resonances}

The investigation of the upper border of the MB island has shown that coexisting MB and RO pulsations appear there. Tuning the injection currents, the frequencies of both oscillations can be adjusted in a wide range. Distinct dynamical phenomena are expected, since the oscillators can dynamically readjust in order to achieve a rational frequency ratio. The amount of frequency readjustment is dependent on the coupling strength between the oscillators. Boundaries between quasiperiodic and locked motion on the torus are organized by saddle-node of limit cycle (SNOLC) bifurcations [55]. In the parameter plane of coupling strength $\varepsilon$ and the frequency ratio $\omega / \omega_{0}$ of the oscillators, the SNOLCs give rise to resonance (also called Arnold) tongues. They indicate the regions of stable $m: n$ frequency locking $(m, n \in \mathrm{N})$.

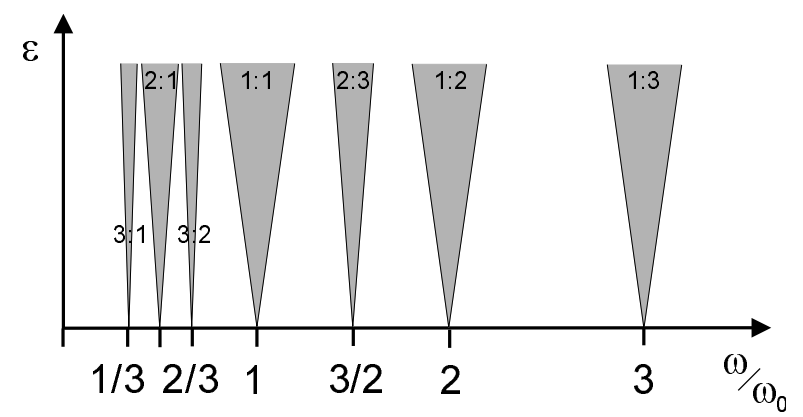

Figure 7.1: Schematic representation of Arnold tongues, the order of locking is indicated. Only locking ratios up to third order are drawn for clarity (after [73], p. 66).

Figure 7.1 schematically illustrates Arnold tongues for different locking ratios in 


\section{The torus regime}

dependence of the coupling strength $\varepsilon$. Only selected ratios of low order are shown. In general, the locking range is largest for 1:1 locking, when both oscillators have the same frequency, and decreases with increasing order of locking. It is obvious that a large number of tongues can occur. For high coupling strength they will overlap already for low rational orders and cause new dynamics.

A comparison of rf spectra for different resonances is shown in Fig. 7.2. For the strong resonances up to 1:4 a small linewidth and similar power of the spectral com-

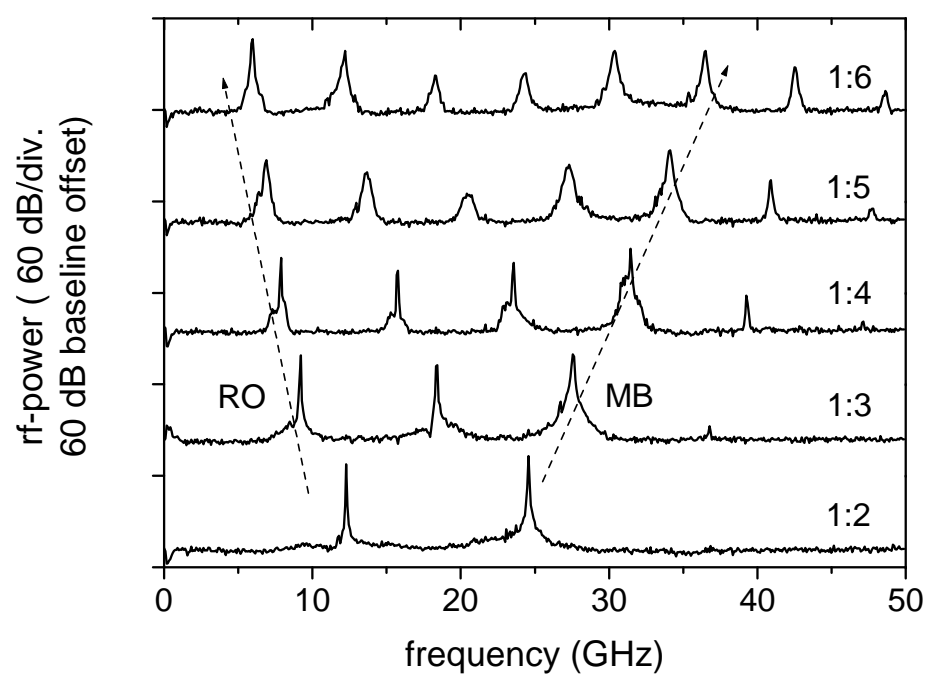

Figure 7.2: Power spectra of 1:2 and higher order resonances. The main RO- and MBcomponents are indicated. Other spectral features are a result of intermixing.

ponents is found. Higher locking ratios show a distinct noise floor around the different spectral lines. Reason for the weaker synchronization is the decreasing modulation response of the carrier density at high MB frequencies. The observation of weaker coupling for high frequency ratios corresponds to a smaller width of the Arnold tongues.

\section{Characteristic behavior of resonances}

Major modifications of RO and MB dynamics are expected due to the resonances. This Section focuses on the onset of locking and the laser behavior in the locked state. The different locking ratios presented in the previous Section were characterized. Since the general phenomena for the different locking ratios were found similar, here a detailed analysis for the 1:2 resonance is given. Results are qualitatively valid for the other resonances.

The evolution of the 1:2 resonance was investigated for a fixed phase current of $I_{P}=26.6 \mathrm{~mA}, I_{A}$ serves as bifurcation parameter. Figure 7.3(a) shows the frequency tuning behavior of the MB oscillator. At the lower border of the pulsation island the frequency is $21 \mathrm{GHz}$. The smooth increase of the rf power in Fig. 7.3(b) indicates the onset of pulsations by a supercritical Hopf bifurcation. With rising feedback, i.e. amplifier current, the MB frequency increases. A large modulation depth is indicated 

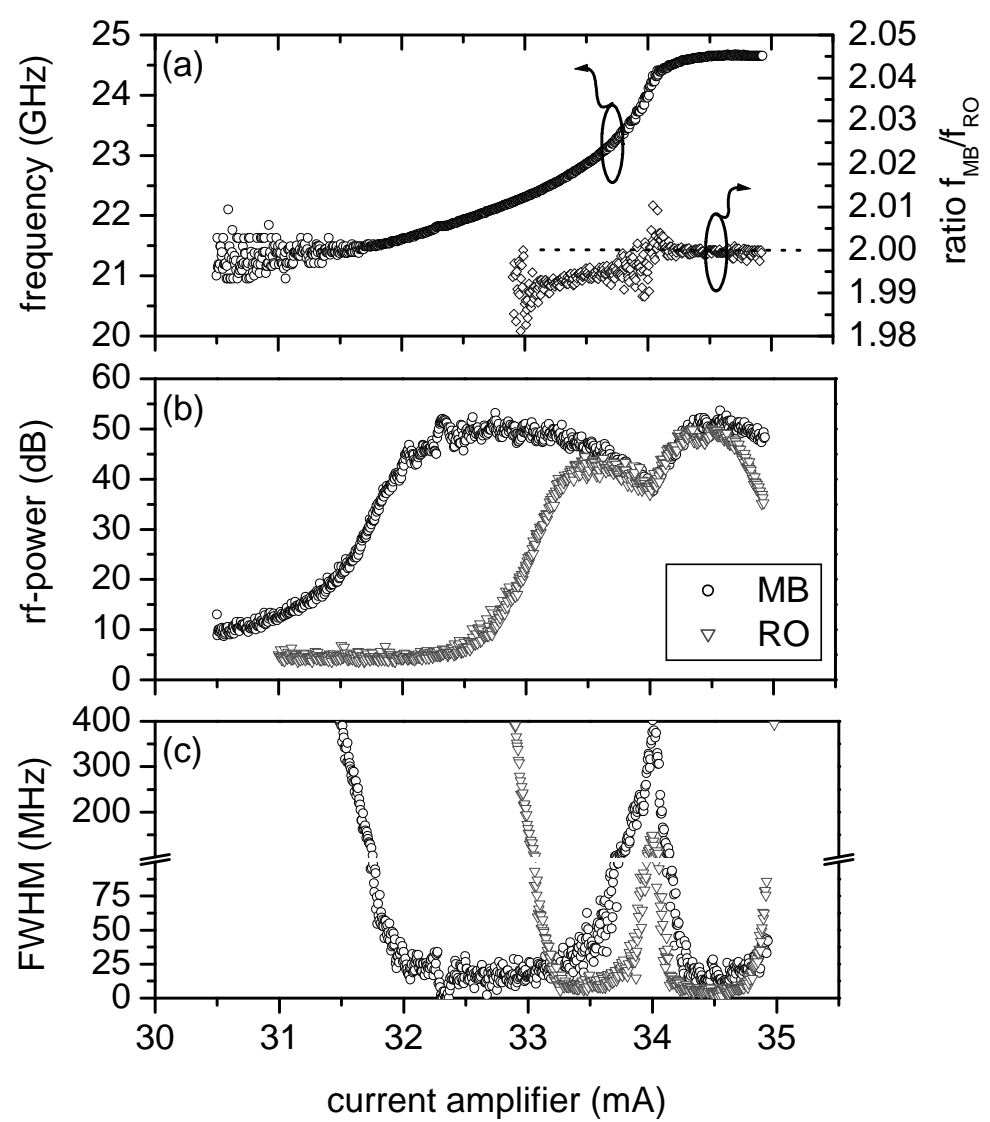

Figure 7.3: Analysis of the 1:2 resonance. The evolution of (a) MB frequency and $f_{M B} / f_{R O}$, (b) the power of both oscillators and, (c) the full-width at half-maximum (FWHM) linewidth of each frequency component is shown (please note the change in scale for (c)).

by the strong rf signal. According to the decreased damping of pulsations a smaller spectral linewidth is observed in Fig. 7.3(c). An additional frequency component due to period doubling (PD) appears at $32.5 \mathrm{~mA}$. Similar as the MB oscillations, the $\mathrm{PD}$ sets on by a supercritical bifurcation. Comparable intensities of both oscillators are found between $33.5 \mathrm{~mA}$ and $34.5 \mathrm{~mA}$ (deviations are within the linearity of the measurement equipment). At $I_{A}=34 \mathrm{~mA}$ a decoupling of the $\mathrm{MB}$ frequency from the amplifier current occurs, and the pulsation frequency remains almost constant in a range of $0.5 \mathrm{~mA}$. In the situation of unlocked oscillators, the MB frequency would increase with feedback strength whereas the RO slows down. This dependency of the pulsation frequencies is suppressed in order to maintain the locked state.

For a deeper understanding of the oscillator interaction the frequency ratio of the pulsations and the linewidth of the power spectra were analyzed (Fig. 7.3(a) and (c)). The FWHM linewidths of the spectra were determined by fitting with a Lorentzian. Two distinct areas are found: below $34 \mathrm{~mA}$ the frequency ratio of the $\mathrm{MB}$ and $\mathrm{RO}$ oscillator converges to a ratio of two, with a lag of the MB oscillations relative to the ROs. Above, the frequency ratio is fixed at exactly two. This plateau corresponds to 


\section{The torus regime}

the synchronization region. A constant ratio of $\mathrm{rf}$ linewidths indicates the entrainment of oscillators already before locking. The frequency ratio remains approximately two in the whole parameter range where both oscillators coexist.

Measurements around $I_{A}=34 \mathrm{~mA}$ in both directions have shown that there is no hysteresis at the transition to the locked state (experimental limitation $\Delta I_{A}=$ $0.001 \mathrm{~mA}$ ). In this parameter range the strong dependence of pulsation frequency on the amplifier current converts any fluctuation, e.g. by spontaneous emission noise, into a large frequency deviation. The measurements with the ESA average over the fast dynamics of the laser system and show the switching due to noise in a broadened linewidth. Once the oscillator system is in a locked state, the linewidths are strongly reduced. This results in a FWHM of $4 \mathrm{MHz}$ for the $\mathrm{RO}$ and $8 \mathrm{MHz}$ for the MB pulsations, respectively. The linewidth increases towards the border of the pulsation island, indicating a destabilization of the 1:2 resonance. However, a cascade of period doublings as a typical scenario leading into chaotic emission is not observed in experiment. This is attributed to the small width in parameter space where the PD cascade occurs. Noise induced fluctuations cause a switching to the next mode, before chaos occurs. The truncation of PD sequences in presence of noise is a well known phenomena [27]. Numerical investigations for the AFL have shown the existence of a PD cascade when spontaneous emission noise is neglected.

The recovery dynamics of the device close to the onset of $\mathrm{PD}\left(I_{A}=32 \mathrm{~mA}\right)$ was investigated to verify that the low frequency pulsations involved in the PD scenario are of RO type. For the perturbation of the laser state optical pulses with a duration of $12 \mathrm{ps}$, a repetition rate of $156 \mathrm{MHz}$, an average power of $5 \mathrm{~mW}$ (in the fiber), and a wavelength of $1550 \mathrm{~nm}$ were injected into the amplifier section of the device. When
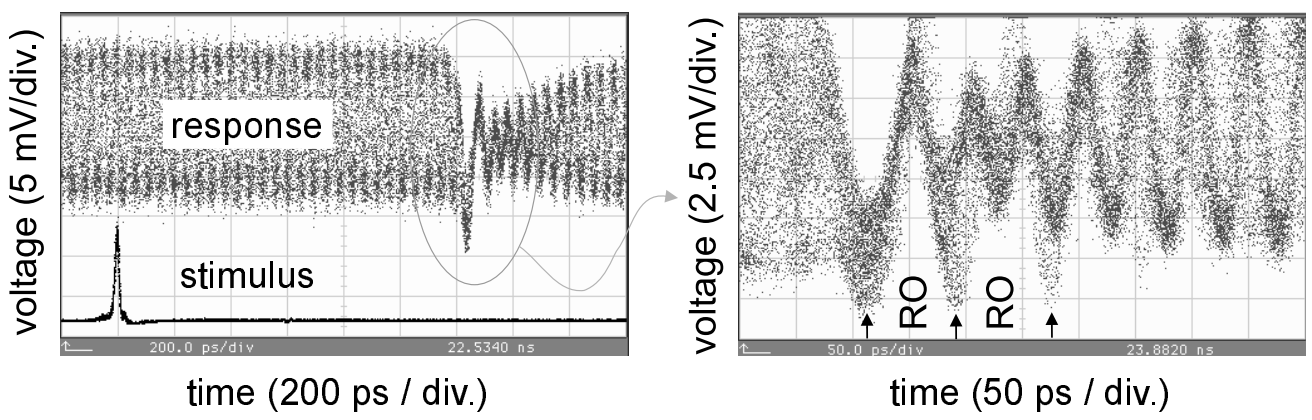

Figure 7.4: Investigation of temporal dynamics close to PD. (left) Stimulus and response of AFL in an overview. The shift of the traces is due to the experimental setup. (right) Detail of response, indicating $\mathrm{RO}$ dynamics.

a pulse enters the amplifier section, the injected light causes an instantaneous carrier depletion due to stimulated emission, equivalent to an abrupt decrease of $I_{A}$. The perturbation recovers with the time constant of the relaxation oscillations. Figure 7.4 illustrates these measurements. The left oscilloscope picture shows the injected pulse (stimulus, lower trace) and the response of the laser (upper trace). ${ }^{1}$ By the periodical

\footnotetext{
${ }^{1}$ Both traces are shifted with respect to each other due to different optical path lengths between
} 
perturbation the MB oscillations are partly synchronized and therefore visible as distinct pulse train on the oscilloscope. The device response (Fig. 7.4 right) shows that the MB trace is superposed by strongly damped relaxation oscillations. The period of the ROs is approximately $90 \mathrm{ps}$, verifying that the low frequency oscillations in the PD area are in fact due to undamped ROs.

\section{Numerical experiments}

The investigation of the PD area has shown that characteristic phenomena for interacting nonlinear oscillators occur in the AFL. In experiment, only the optical output of the device is accessible. Carrier density investigations could provide a more detailed insight to the dynamics. However, parasitic capacitances prevent the measurement of junction voltage oscillations at the frequencies of $\mathrm{RO}$ and $\mathrm{MB}$ pulsations (several $\mathrm{GHz}$ to several tens of $\mathrm{GHz}$ ).

As an alternative, numerical experiments in the torus regime using LDSL were performed. They allow for a deeper understanding of the underlying physics. Operation points for resonances are summarized in Tab. 7.1; the same laser parameters as in Section 4.5 were used. Figure 7.5 shows the results for MB pulsation, PD and different tori. Phase space projections of the carrier densities in both active sections are drawn. Additionally, Poincaré sections of the phase space trajectory specify points of local power maxima. ${ }^{2}$ LSHB was taken into account for the simulations, therefore only the carrier densities at the center of the active sections are plotted. The diagrams summarize the device dynamics over $10 \mathrm{~ns}$, except for the quasiperiodic torus, where only 2 ns are shown for clarity.

\begin{tabular}{|c|c|c|c|c|}
\hline scenario & $I_{D F B}(\mathrm{~mA})$ & $\phi_{P}(2 \pi)$ & $I_{A}(\mathrm{~mA})$ & $f_{M B}$ \\
\hline \hline MB & 70 & 0 & 19 & 25.78 \\
$1: 2$ & 70 & 0.4 & 15.4 & 23.93 \\
$1: 3$ & 70 & 0.2 & 17.8 & 25.01 \\
1:4 & 70 & 0.7 & 23.6 & 28.62 \\
1:5 & 70 & 0.2 & 30.5 & 32.67 \\
quasiperiodic & 70 & 0 & 33.4 & 33.68 \\
break-up & 70 & 0 & 35.1 & - \\
\hline
\end{tabular}

Table 7.1: Operation points for investigations in the torus region. Thermal detuning in the amplifier section and the dependency of losses on $\phi_{P}$ were neglected.

Small excursions in $n_{D F B}$ and $n_{A}$ characterize the carrier dynamics for the beating pulsation. The reason is the strong damping for frequencies above $f_{R O}$ [69]. Oscillations of the carrier densities in the active sections are phase shifted due to the propagation delay of the optical peak power. This gives rise to the non-diagonal trajectory. Moving counterclockwise around the closed curve corresponds to one period of the MB pulsation. A comparison of carrier density excursions in amplifier and DFB section shows

the signal sources and the oscilloscope.

${ }^{2}$ Other Poincaré sections, e.g. at a fixed carrier density in one of the active sections do not capture the full dynamics due to the different carrier density excursions from RO and MB. 

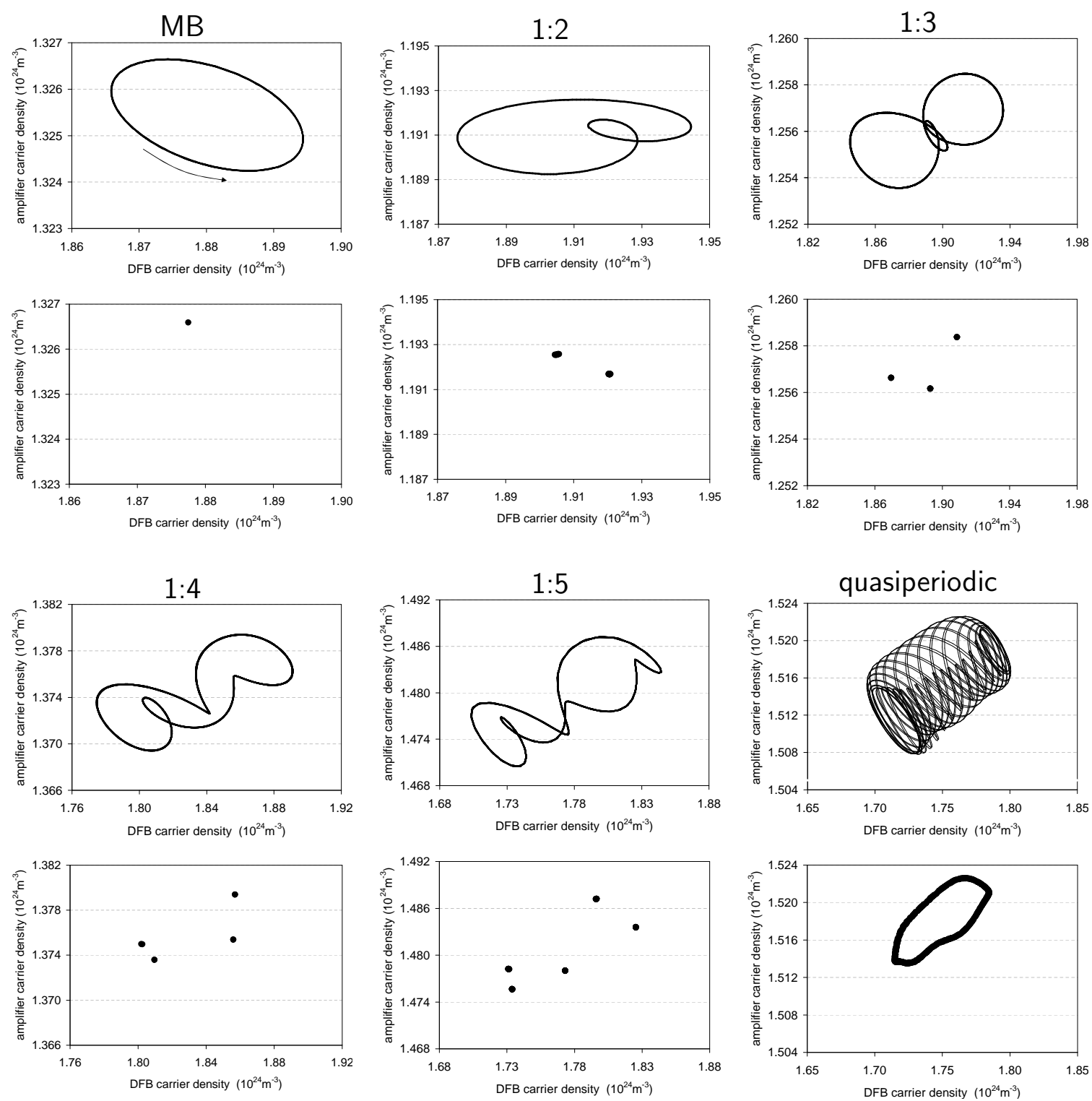

Figure 7.5: Phase space projections in the plane of $n_{D F B}$ vs $n_{A}$ and Poincaré sections for the indicated scenarios. Poincaré sections are drawn with respect to local maxima of output power.

that variations in $n_{A}$ are by approximately one order of magnitude smaller than in $n_{D F B}$.

When additional ROs become undamped, an in-phase motion - i.e. oriented along the bisecting line in the $\left(n_{D F B}, n_{A}\right)$-plane - of both carrier densities is superposed to the MB trajectory. The limit cycles of resonant states become closed orbits and the fast MB pulsations cause excursions around the RO trajectory. The DFB carrier density modulation by the relaxation oscillations is in the order of $0.1 \times 10^{24} \mathrm{~m}^{-3}$, again depending on the frequency of the RO component. 
Besides the obvious difference in frequency, the phase space trajectory also gives details about the temporal characteristics of the two oscillation types. MB pulsations are due to the beating of longitudinal modes and therefore cause sinusoidal excursions of the carrier density. The non-equidistant excursions of the MB pulsations on the limit cycle show, that the carrier build-up (lower branch of torus) is slow in comparison to the pulse emission (upper branch). This asymmetry induces strong harmonics in the power spectra.

In the general case that $\mathrm{RO}$ and $\mathrm{MB}$ pulsations have a non-integer frequency ratio, a quasiperiodic orbit occurs and the trajectory densely fills the torus in phase space. The example in Fig. 7.5 (lower right) shows a trajectory for a pulsation with a MB frequency of $33.68 \mathrm{GHz}$ and a $\mathrm{RO}$ frequency of $6.28 \mathrm{GHz}$ (only 2 ns illustrated).

\subsection{Break-up of torus}

Quasiperiodic or locked oscillations occur in the torus region, the respective behavior is organized by the Arnold tongues. When different Arnold tongues start to overlap, the dynamic situation becomes unstable and the torus starts to break up. The torus break-up is another well known route to chaos besides the PD cascade. It is therefore interesting to investigate the border of the torus region with respect to chaotic emission.
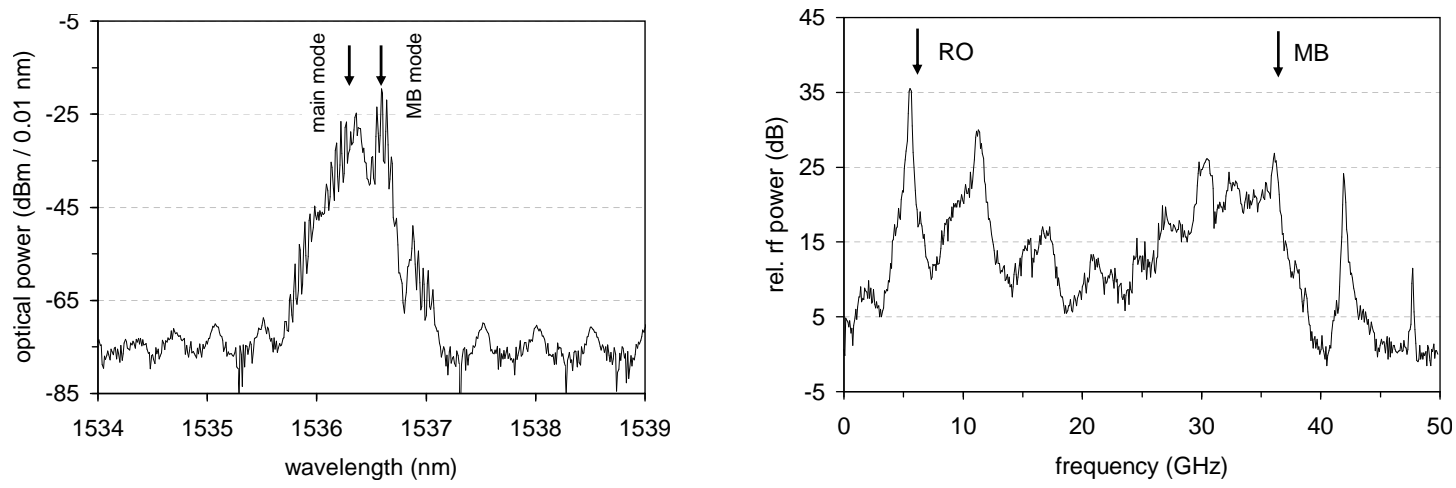

Figure 7.6: Experimentally observed break-up of torus at $I_{A}=49.88 \mathrm{~mA}$. The left panel shows the optical spectra, arrows mark the MB modes. Spectral features of the pulsation extend over several longitudinal modes. The right panel displays the corresponding power spectrum. As a consequence of the chaotic behavior a broadened spectrum is observed.

Indeed, the border of the torus area depicted in Fig. 6.7 shows irregular behavior. Typical spectra from the overview are presented in Fig. 7.6. The spectra are broadened, but still contain the main features of RO and MB pulsations. In the optical spectrum, a splitting of the coexisting beating modes due to the RO instability can be observed. Corresponding peaks occur in the power spectrum. A significant interpretation of optical and electrical spectra of chaotic behavior is difficult, as they only 


\section{The torus regime}

give an averaged description of the dynamics. With the presently available technology, a real-time measurement of the temporal characteristics is not possible because of the involved frequencies. Oscilloscopes covering the range above $5 \mathrm{GHz}$ are based on sampling techniques and therefore cannot deliver a continuous time trace.

Alternatively, simulations were performed to gain insight into the break-up dynamics. Poincaré sections in the $\left(n_{D F B}, n_{A}\right)$ carrier density plane for local optical power maxima serve as illustration. Characteristic scenarios during the torus break-up are
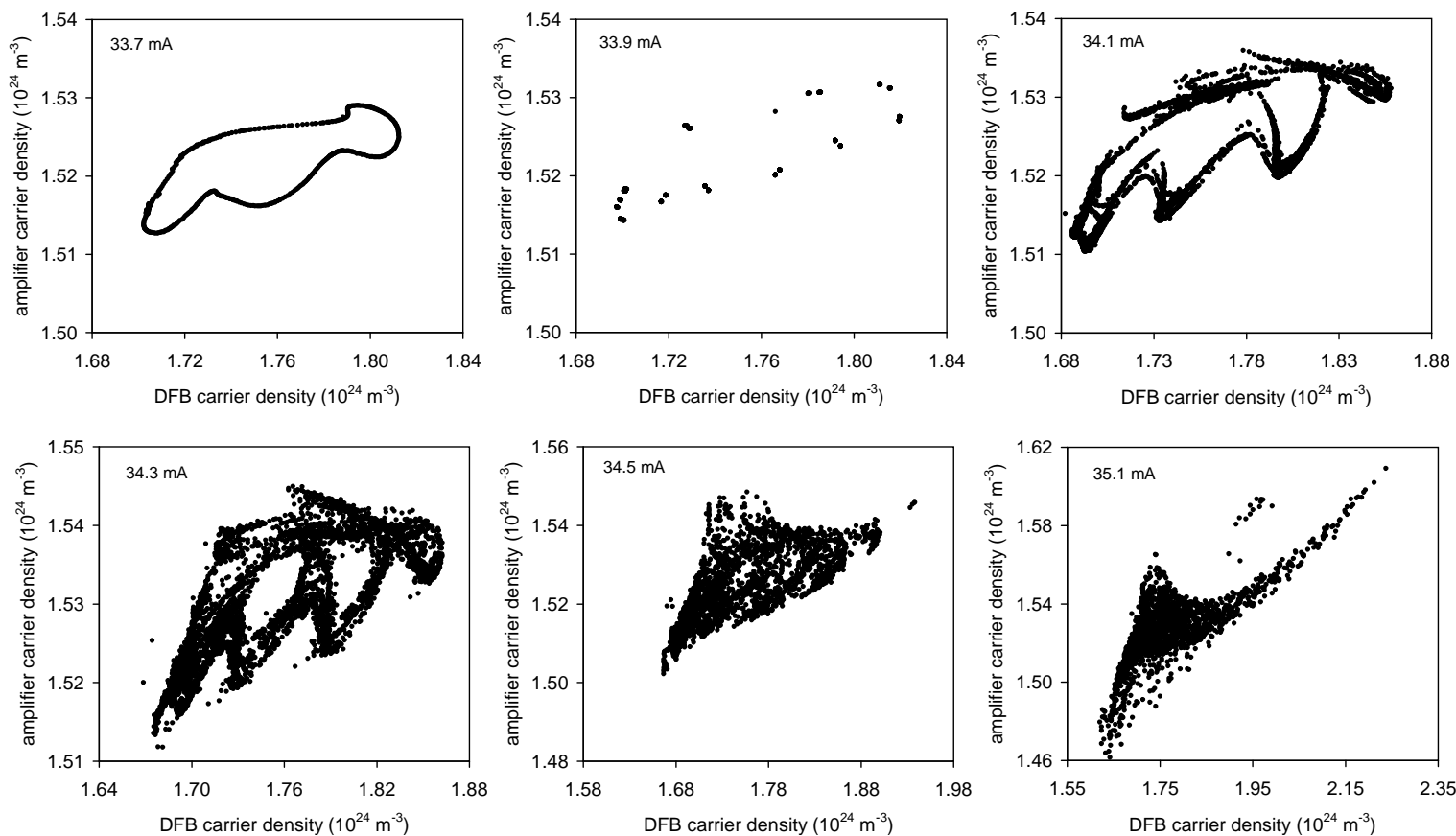

Figure 7.7: Poincaré sections showing the break-up of a torus. $I_{A}$ values are denoted in the panels. Scales are changing due to the expansion of the chaotic attractor.

summarized in Fig. 7.7. Starting point for the simulations is the quasiperiodic orbit illustrated in Fig. 7.5. From here, the torus break-up is investigated by increasing the amplifier current in small steps, i.e. the operation point approaches the border of the torus region. Already a slight increase of the amplifier current shows that the originally smooth Poincaré section becomes wrinkled. Rational frequency ratios give rise to higher order resonances, showing up as discrete points in the Poincaré sections $\left(I_{A}=33.9 \mathrm{~mA}\right)$. Towards the border of the pulsation island the torus starts to break up. The Poincaré sections become more and more fractal, until the original structure of the torus attractor is lost. During the break-up, the attractor undergoes a strong expansion in phase space. Such an expansion of the attractor is commonly referred to as 'explosion' of a chaotic set or interior crisis [77]. A similar quasiperiodic route to chaos has been shown for the long cavity regime and the scenario of low frequency fluctuations $[65,78]$. 


\subsection{Boundary crisis}

The phase space configuration underlying the torus break-up can be reconstructed from the temporal dynamics. Figure 7.8 illustrates the output power and the corresponding projection of the trajectory in phase space at $I_{A}=35.1 \mathrm{~mA}$. The power fluctuations of the device output still show the signature of RO and $\mathrm{MB}$ oscillations which underly the chaotic state. The power spectrum is similar to the experimental results given in Fig. 7.6, however, with less distinct spectral features. The phase space projection of the trajectory in Fig. 7.8 shows the ruin of the torus attractor. Trajectories with large excursion in the carrier density emerge from the destabilized attractor. They correspond to periods of suppressed laser emission, where the carrier density in the active sections can build up, before an optical burst is emitted. The associated decrease in carrier densities returns the device into a trajectory along the chaotic attractor. An example for the carrier build-up, a burst and the subsequent dynamics is highlighted in Fig. 7.8 for comparison.
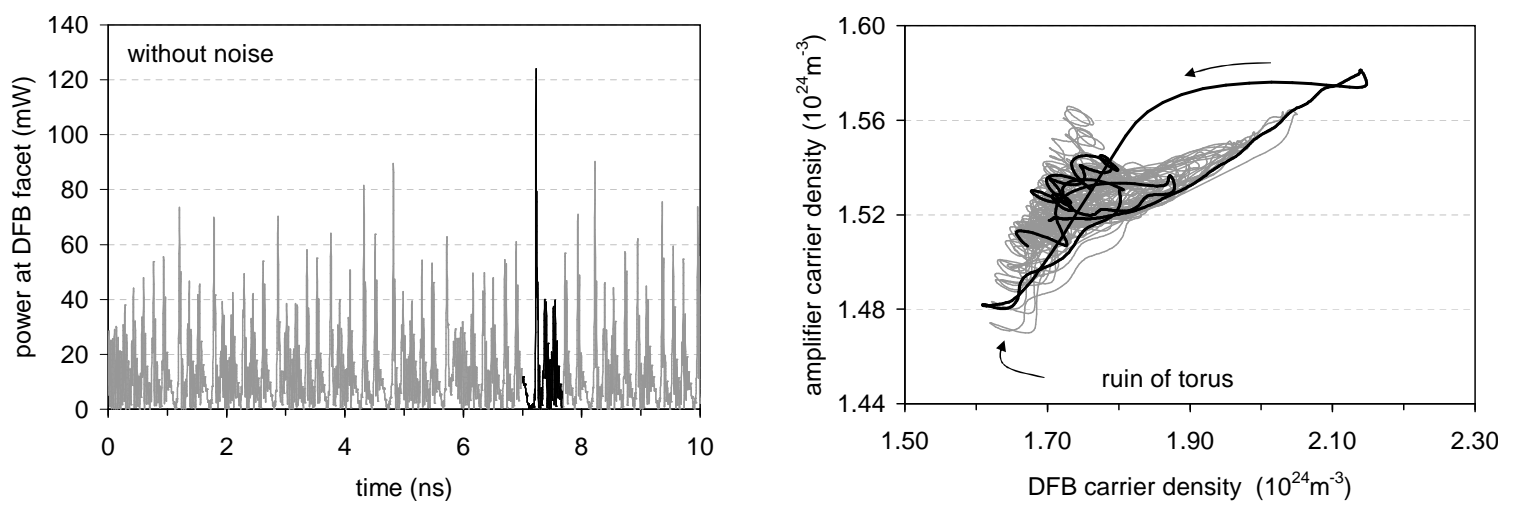

Figure 7.8: Torus break-up dynamics. The left panel shows the time trace of device output, the right panel illustrates the corresponding phase space projections.

The observation of suppressed laser emission clearly indicates that a saddle is involved in the dynamics. Trajectories coming close to the saddle point are strongly slowed down and long periods of suppressed emission occur. The situation is therefore similar to a homoclinic bifurcation. Only the regular limit cycle is replaced by a chaotic attractor, which annihilates in a homoclinic loop.

A sketch of the dynamical scenario in phase space is given in Fig. 7.9. The chaotic attractor comes close to a saddle point which now lies in the attracting region. From the saddle one unstable manifold directs back into the attractor, whereas the other leads to a different state of the system, e.g. lasing on another mode. Once the chaotic attractor collides with its attracting region, it suddenly looses stability. All trajectories now lead to the new attracting state. Such a phenomenon is commonly referred to as boundary crisis [39].

The dropouts in laser emission already indicate trajectories coming close to the saddle point. In this situation, small changes of the operation point can cause the 


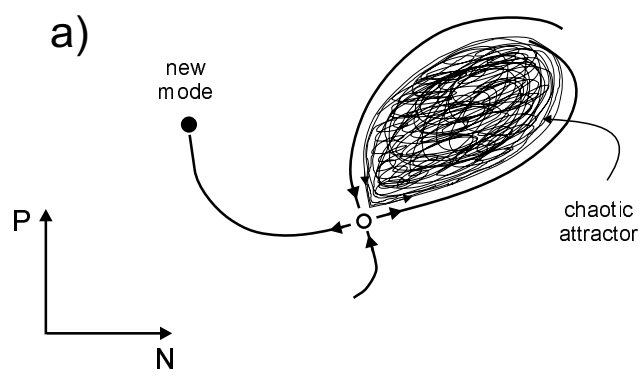

b)

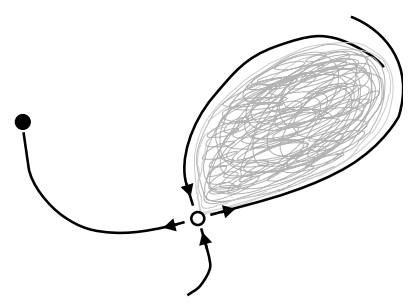

Figure 7.9: Phase space scenario giving rise to a boundary crisis. (a) A chaotic attractor and a saddle approach each other. (b) After the collision of saddle and chaotic attractor all trajectories point towards the new state, chaos has lost stability.

collision of the chaotic attractor and the saddle. To investigate the annihilation of the chaotic attractor, the simulations were repeated. Instead of changing the operation conditions, only spontaneous emission noise was included in the computations.

Figure 7.10 shows a representative time trace in presence of noise. The temporal characteristics due to the chaotic attractor remains unchanged. As soon as noise causes the collision of attractor and saddle, the laser makes a sudden transition to another stable state. In the investigated parameter range this is a MB pulsation with small amplitude. The disappearance of the chaotic attractor gives the clear evidence for a boundary crisis scenario.
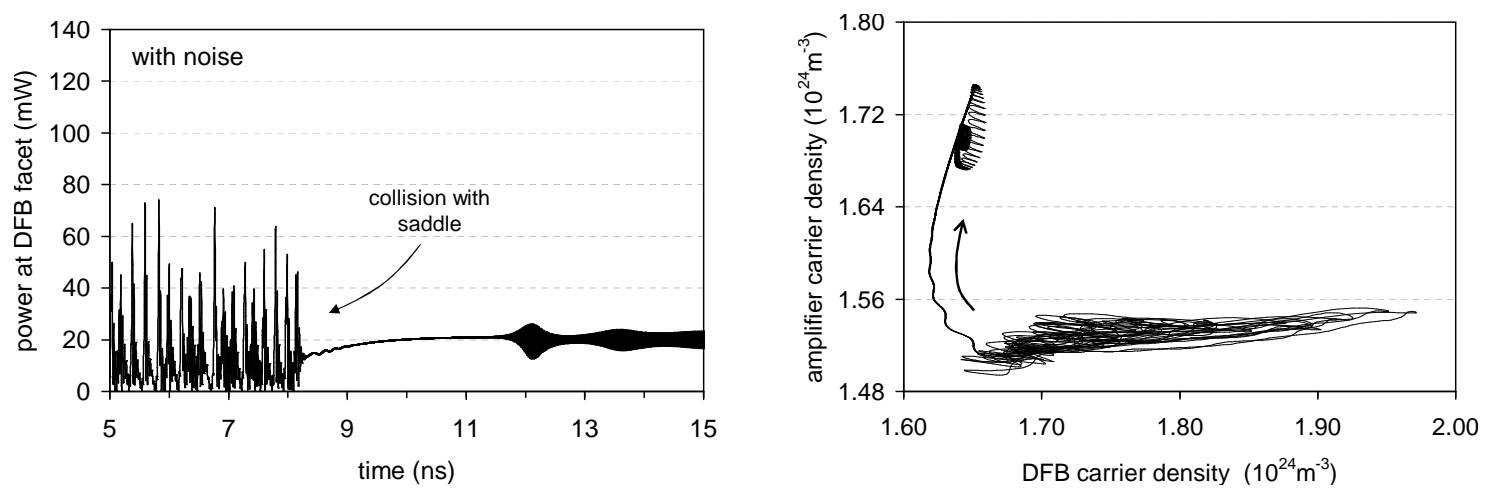

Figure 7.10: Torus break-up dynamics with spontaneous emission noise. Time trace and phase space projection are shown. Noise causes the collision of the chaotic attractor with a saddle, inducing the transition to a different laser state.

The observed impact of noise at the island border explains the small range where irregular tori can be observed in experiment. Whereas the breakup of the torus in simulation develops over a range of more than $1 \mathrm{~mA}$, in experiments only a width of $0.5 \mathrm{~mA}$ can be observed. The reason is spontaneous emission noise which induces a boundary crisis before reaching the stability border of the unperturbed chaotic attractor. Hysteresis prevents the noise induced switching between the two states for the investigated device. 
Once the hysteresis becomes small, e.g. at higher feedback strengths (cf. Fig. 6.3) or for modified device geometries, noise can switch between adjacent mode-pairs. A study of the phenomena arising from closely spaced bifurcations under the impact of external noise could be interesting. 


\section{Chapter 8}

\section{Impact of noise}

The most characteristic phenomena arising in the AFL were already presented. This Chapter gives selected examples where internal noise modifies the generic behavior. Noisy precursors are demonstrated as indicator of impending instabilities. Furthermore, noise induced irregular dynamics at the transition between RO and MB areas is demonstrated.

\subsection{Noisy precursors}

Noise is a phenomenon present in any semiconductor laser due to spontaneous emission. Modifications of the dynamics due to noise have already been demonstrated. Examples are the onset of pulsations which is smoothed by noise and the truncation of a period doubling (PD) cascade. Another generic phenomenon in nonlinear systems with noise are noisy precursors of bifurcations [93]. Precursors show up as bumps in the power spectrum, when the investigated operation point is near to a bifurcation. In such a state, the system is almost unstable and, hence, more susceptible to noise than far from the bifurcation. The frequency of the noise enhancement indicates the frequency of the instability that will occur. The nearer the bifurcation, the higher the noise intensity of the precursor. ${ }^{1}$ In this way, noise can indicate unstable or 'virtual' objects.

In the AFL, noisy precursors can be observed for RO and MB pulsations, when the operation point is close to the corresponding Hopf bifurcations. Strongly damped oscillations already indicate the pulsations far from the bifurcation point (cf. Fig. 6.1). A more complex example is given in Fig. 8.1, displaying a blow-up of regime IV in Fig. 6.1. The situation shows the continuous metamorphosis of precursors close to a $1: 3$ resonance.

For a more detailed discussion of the experimental results, the frequency and amplitude of MB pulsation and precursor are summarized in Fig. 8.2. The respective values are displayed for the MB oscillation and the low frequency spectral component of the precursors. As expected, the frequency of the MB pulsation increases with $I_{A}$. In the

\footnotetext{
${ }^{1}$ Precursor intensity also scales with the strength of noise. In a semiconductor laser it is given by the spontaneous emission, which remains fixed at its threshold value.
} 


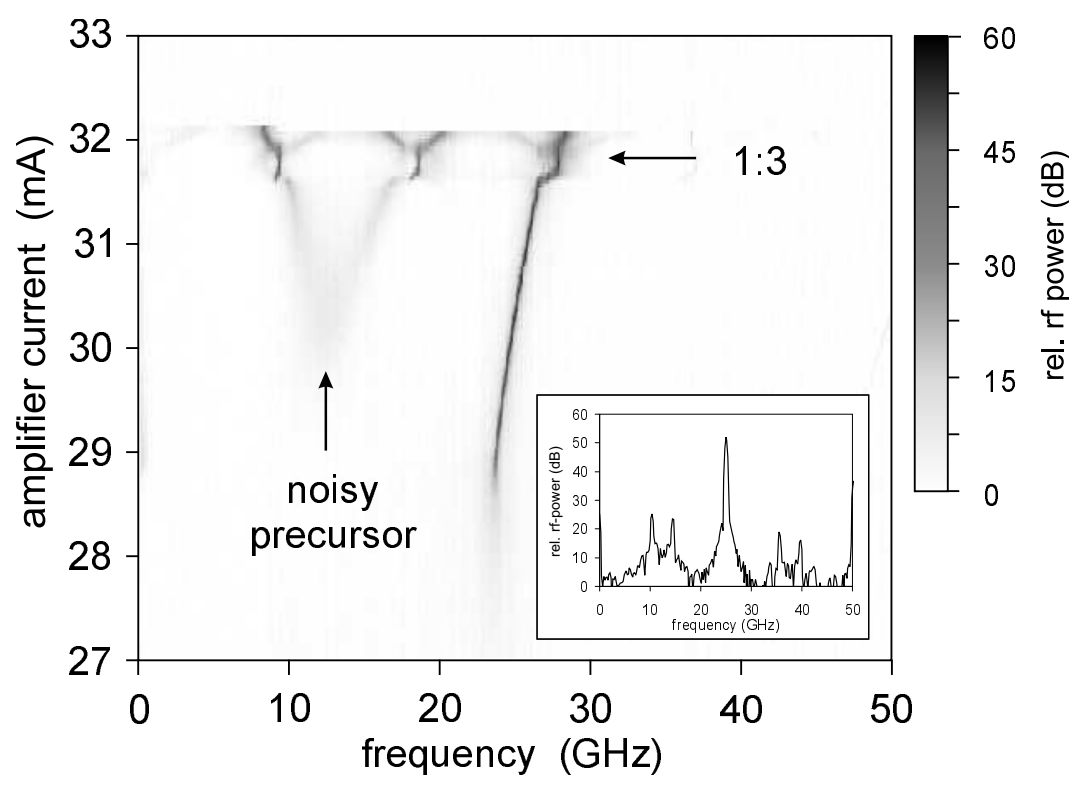

Figure 8.1: Evolution of power spectra close to a torus bifurcation. Noisy precursors of a $\mathrm{PD}$ and a subsequent torus bifurcation are observed. The inset shows a simulated spectrum, qualitatively confirming the measurement results.

$I_{A}$-range where the precursor grows out of the noise floor of the ESA, the frequency ratio between the $\mathrm{MB}$ pulsation and the precursor is two, thereby characterizing the precursor of a PD bifurcation. Since this precursor does not actually induce the PD bifurcation, such a scenario is called 'virtual' PD. The noisy signature of the virtual PD continuously changes into the precursor for a Hopf bifurcation by splitting up symmetrically around the PD location. Controlled by $I_{A}$, the frequencies gradually approach a 1:3 ratio. The intensity of the precursors increases, confirming the approach to the bifurcation point. Here, a similar jump into the locked state as for the 1:2 resonance is observed. Towards the island border, synchronization is lost and a quasiperiodic pulsation occurs. Simulations of the experimentally observed scenario have been performed. A power spectrum from simulations is shown in the inset of Fig. 8.1. It qualitatively verifies the measured spectra. Similar observations for PD and 1:3 precursors have been made in experiments with directly modulated semiconductor lasers in [49].

Both, noisy precursors and the metamorphosis of attractors are generic phenomena demonstrated theoretically for a large number of nonlinear systems like the driven damped pendulum and the Lorenz equations [93]. The verification of these phenomena in the AFL shows that the non-deterministic behavior of this laser type follows similar laws as low-dimensional systems. Hence, the vast knowledge for these systems can be applied in the investigation of AFL dynamics. 

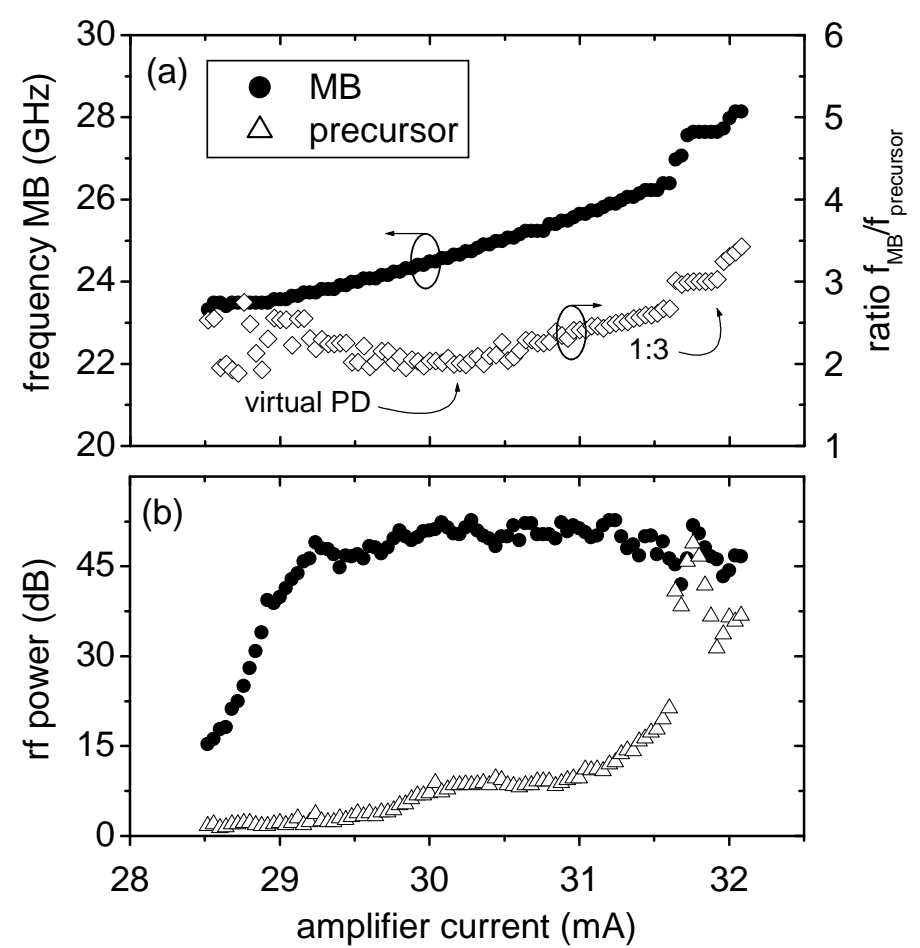

Figure 8.2: Analysis of the precursor scenario shown in Fig. 8.1. (a) Frequency of MB and frequency ratio of $\mathrm{MB}$ and precursor. (b) Spectral peak power of MB and precursor/resonance.

\subsection{Noise induced irregular dynamics}

The basic pulsation types in the AFL are undamped ROs and MB oscillations. In the idealized case of a system without noise, which was investigated in the bifurcation analysis, sharp transitions between the pulsation regimes occur. The transitions are modified by noise, which can either induce jumps or smooth the behavior at the bifurcation borders.

The upper part of the RO island has been investigated with respect to noise induced modifications. Here, numerous bifurcations are closely spaced and allow different transitions. From the bifurcation analysis an area of undamped ROs is expected. These pulsations can undergo PD and saddle-node of limit cycle bifurcations (SNOLC, dotdashed line) only when crossing the bifurcation border from the inside of the pulsation island. In the experiments, these bifurcations should either lead to discontinuous jumps of the frequency (SNOLC) or an additional spectral component at half frequency (PD). Pulsations are expected to end in homoclinic bifurcations at the right island border.

Figure 8.3 gives a measurement overview for the frequency of the main spectral component in the transition region. Spectra were recorded for decreasing $I_{P}$. Thus, all observed features lie left to the saddle-node bifurcation, which accordingly coincides with the right island border. Furthermore, by performing the measurement in backward $I_{P}$-direction, the MB pulsations are absent due to hysteresis (above $I_{A}=17.5 \mathrm{~mA}$ ). 


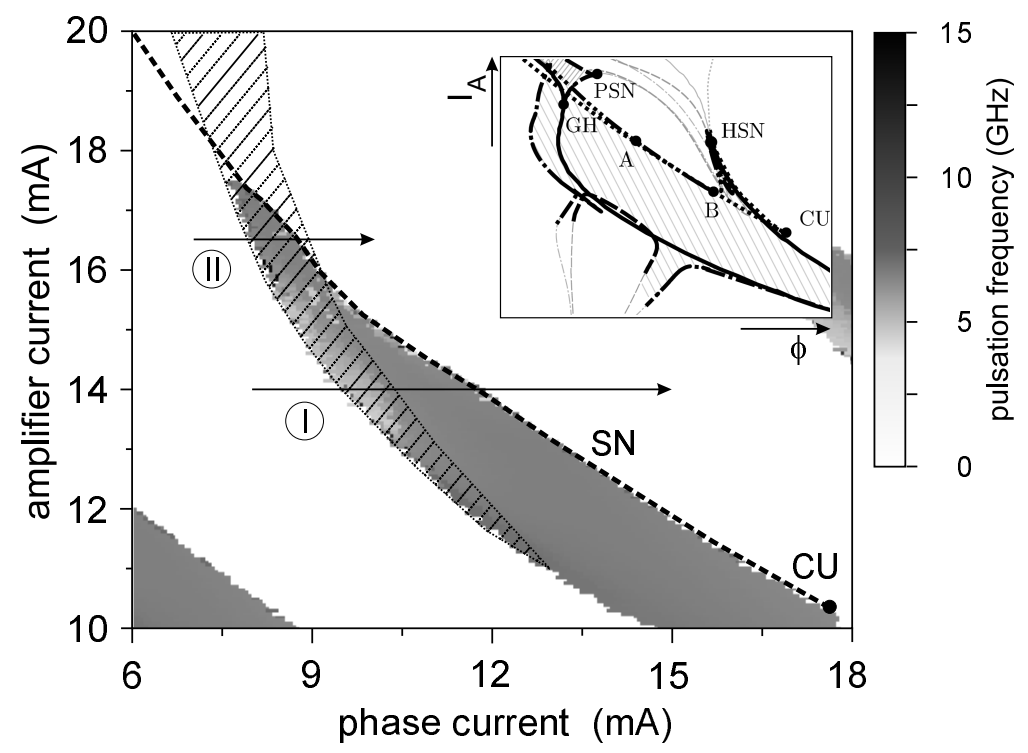

Figure 8.3: Detail of dynamics in the transition region from RO to MB pulsations. The main frequency of the power spectrum is indicated (spectral peaks exceed noise floor by more than $10 \mathrm{~dB}$ ). The hatched area gives the location of strongly damped MB pulsations coexisting with ROs. The inset shows the equivalent section of the bifurcation diagram.

The strongly varying gray scale towards the tip of the island indicates deviations of the device dynamics from the expected regular RO pulsations.

For a clarification of the experimental situation power spectra at two different amplifier currents were analyzed. In contrast to the overview measurement, the power spectra were recorded for increasing $I_{P}$ in order to detect differences induced by the direction of parameter variation. Results for $I_{A}=14 \mathrm{~mA}$ are shown in Fig. 8.4. At the left island border a broad spectrum with a central frequency of $6 \mathrm{GHz}$ is observed. Here, the laser emission is unstable and switches between $\mathrm{cw}$ and pulsating state. The inset of Fig. 8.4 illustrates the switching dropouts during a frequency sweep of the spectrum analyzer $(\sim 100 \mathrm{~ms})$. Towards the center of the pulsation island this irregular state evolves into a regular pulsation showing distinct frequency components. In addition to the fundamental frequency at $9 \mathrm{GHz}$, a spectral component with $f_{R O} / 2$ appears, indicating a PD bifurcation. A noisy transition to the adjacent $\mathrm{cw}$ state is observed at the right island border. This demonstrates absent or very small hysteresis due to the coincidence of homoclinic and SN bifurcation. Both, the observation of an onset without hysteresis at the subcritical Hopf border and the appearance of $\mathrm{PD}$ in measurement direction clearly indicate different behavior than predicted by the bifurcation analysis. We attribute these modifications to the presence of noise.

Increasing the feedback strength, the regular pulsations disappear. Typical power spectra towards the tip of the RO island are illustrated in Fig. $8.5\left(I_{A}=16.5 \mathrm{~mA}\right)$. In the pulsation island, a strong broadening of the spectra around the characteristic $\mathrm{RO}$ frequencies is observed. This shows the irregularity of the device output in this parameter range. Compared to the measurements at lower $I_{A}$ the width of the unstable 


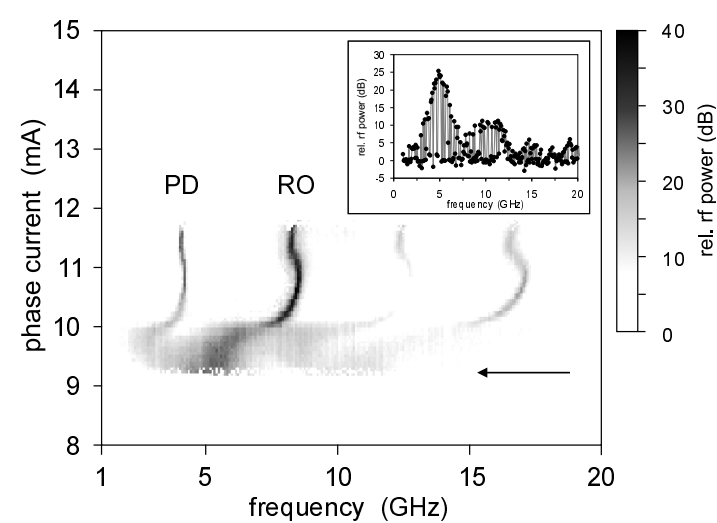

Figure 8.4: Spectral intensity along I in Fig. 8.3. The inset shows the rf spectrum at the left border of the pulsation area (marked by arrow).

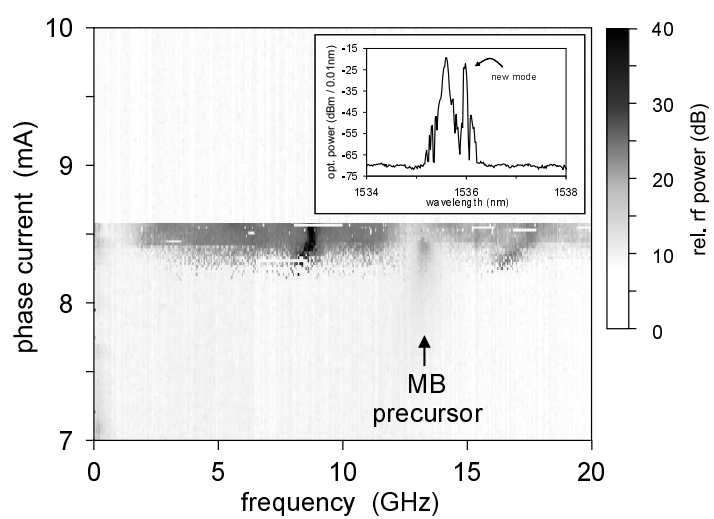

Figure 8.5: Spectral intensity along II. A MB precursor occurs at the left island border. At the right border, a new mode coexists with the pulsating state (see optical spectrum in the inset).

onset region is increased. Intermittent lasing of the adjacent mode occurs at the right island border (inset in Fig. 8.5).

A reason for the more complicated behavior than expected from the bifurcation diagram is the coexistence of a precursor of the MB pulsation. Already before the onset of irregular dynamics, a stable MB precursor with smoothly increasing intensity can be observed (Fig. 8.5). A detailed investigation of the transition region with respect to MB precursors gives the hatched area in Fig. 8.3. Frequency as well as intensity of the precursor increase with the amplifier current, allowing the identification of the MB origin. The area where MB precursors are found coincides with the destabilization region of $\mathrm{RO}$ pulsations. This indicates that the irregular behavior is due to the coexistence of both oscillator types. It has been demonstrated for the torus scenario in the MB regime that coexisting oscillators are a possible source of irregular dynamics. The same situation arises here, however, with RO oscillations and a concurrent MB precursor which are both acting in the same frequency range. The additional PD and SNOLC bifurcations further complicate the dynamical constellation here. Simulations of the behavior in the transition region for a slightly modified AFL geometry in [81] have shown similar results regarding PD and irregular dynamics.

Besides the demonstration that new phenomena are being introduced by noise, this investigation also shows that the bifurcation analysis presented in Section 5.1 cannot cover all subtle details of dynamics. This is already clear from the simplifications made for the bifurcation analysis and the limitation to co-dimension one bifurcations. Noise induces simultaneous fluctuations of several device parameters, therefore more complex bifurcations can occur. Nevertheless, already the location of the basic bifurcation types allows to predict where the possible impact of noise is high. 


\section{Chapter 9}

\section{Locking of AFL dynamics}

The internal dynamics of the AFL has been the focus of this work. External perturbations lead to a modification of the dynamics. With respect to the technical application of the AFL periodic perturbations are of main interest. This Chapter demonstrates that pulsations can be locked to external optical signals. The basic characteristics of the synchronization process are introduced and locking is demonstrated for MB pulsations as well as for a PD scenario.

\subsection{Locking of regular pulsations}

External perturbations - regular or irregular - have been neglected in the investigations so far. To interact with the internal device dynamics, a perturbation has to couple to the carrier density. For the AFL this can be achieved in two ways. The straightforward method is a direct modulation of the injection current of the active sections. Since the employed laser hetero-structure and the bonding wires have large capacitances, a direct broadband modulation is very inefficient and requires strong rf-signals (in the order of $1 \mathrm{~W}$ ). The injection of optical signals is an alternative method of coupling high frequency perturbations to the carrier density of the laser.

Optical locking can be achieved in two different ways. Coherent injection employs an external wavelength exactly matched to the lasing mode. The device dynamics is significantly modified already by very low injection power $(\mu \mathrm{W})$. Due to interference effects, the polarization of the injected signal has to be controlled. ${ }^{1}$ Another possibility is non-coherent injection. Here, the injected signal can be positioned arbitrarily within the gain spectrum of the laser. If the gain medium in the laser shows equal TE and TM gain, the impact of fluctuating polarization can be neglected. The drawback of incoherent locking is that a locking power in the $\mathrm{mW}$-range is required. Additionally, large values of the average injected power may shift the operation point of the device.

For the application of AFL dynamics mainly the locking to periodic signals is of interest. A special case are data signals, where distinct pulses are missing in order to

\footnotetext{
${ }^{1}$ Actually, for coherent locking to periodic signals two locking processes occur: the optical wave as well as the periodic modulations can synchronize.
} 
(a)

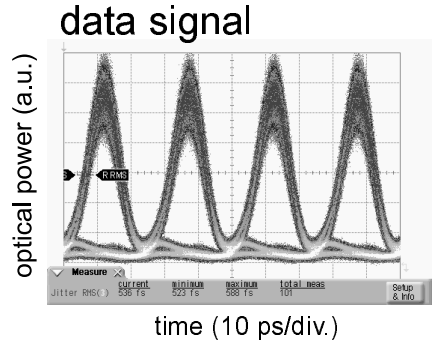

(b)

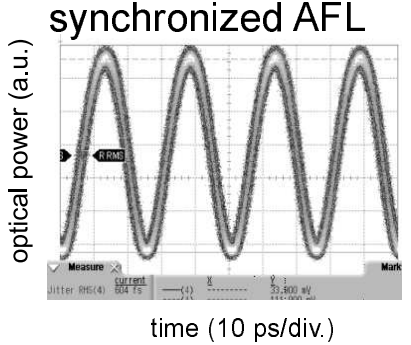

(c) rf-spectra

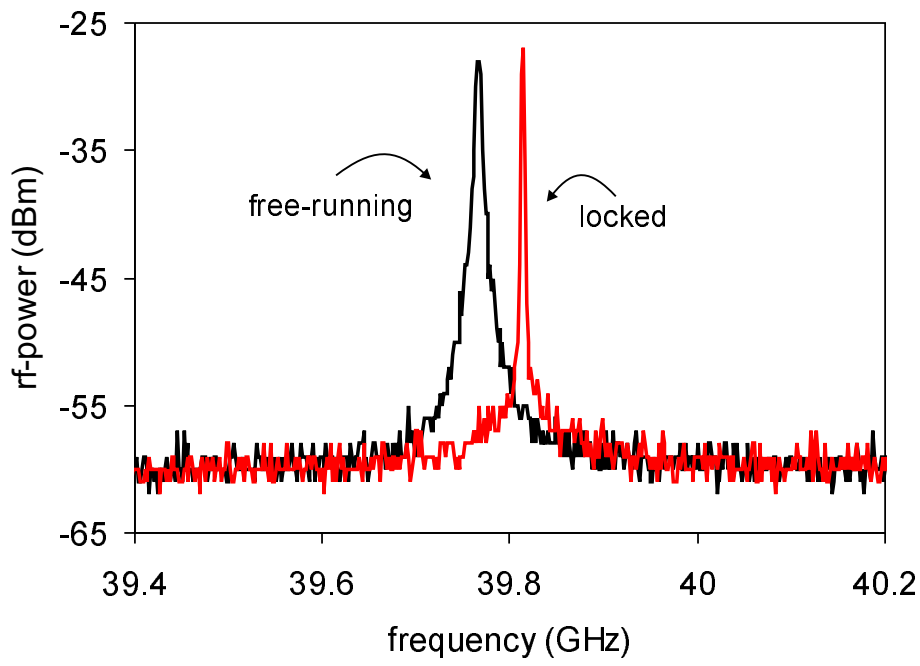

Figure 9.1: Locking to periodic signals. (a) Injected data signal, $39.81 \mathrm{Gbit} / \mathrm{s}$, PRBS $2^{31}-1$. (b) Pulse trace of synchronized AFL. (c) Corresponding power spectra of MB pulsation in free running and locked state.

encode information. An important functionality required for signal processing is clock recovery, i.e. the extraction of the exact frequency and phase of bit streams. This allows a clocked processing of optical data signals without conversion from the optical into the electrical domain.

Figure 9.1(a) shows an optical signal with a data rate of $39.81 \mathrm{Gbit} / \mathrm{s}$, triggered to a frequency of $39.81 \mathrm{GHz} / 4 .^{2}$ The wavelength is adjusted to $1550 \mathrm{~nm}$. Using this data signal, the locking behavior of a MB pulsation was investigated. For the extraction of the clock signal, a free running pulsation with a frequency close to the incoming data rate is adjusted $\left(I_{D F B, P, A}=70,8,75.4 \mathrm{~mA}\right)$. The data signal is injected into the amplifier section of the AFL (typical locking power $\sim 1 \mathrm{~mW}$ ). Since the wavelength of the data signal lies within the gain bandwidth of the amplifier, the injected light is amplified and causes a modulation of the carrier density. The periodic modulation of the carrier density synchronizes the free running pulsation.

Important for the functionality of clock extraction is that once the pulsation is synchronized, the locked state is maintained over sequences where only zeroes are injected. Phase deviations due to spontaneous emission noise are reset by subsequent pulses (ones). In this way, the clock of the injected data signal is recovered with respect to frequency and phase. The fixed phase relation of locking signal and the recovered clock allows the monitoring of the pulse trace emitted by the AFL with a sampling oscilloscope (here an Agilent 86100 was used). Figure 9.1(b) depicts a locked MB oscillation, illustrating the sinusoidal shape of the beating pulsation. The timing jitter

\footnotetext{
${ }^{2}$ The statistics of real data signals is simulated by pseudo-random bit sequences (PRBS). These sequences contain all possible combinations of zeros and ones. Here a pattern length of 31 bits is used, commonly referred to as PRBS $2^{31}-1$.
} 
of the recovered clock signal is $600 \mathrm{fs}$, i.e. in the same order of magnitude as for the injected data signal. Frequency spectra of free running and locked state are shown in Fig. 9.1(c) for comparison. The locking is indicated by the adjustment of the free running pulsation frequency to the injected data rate and a narrowing of the spectral line due to the reduction of phase fluctuations.

\section{Locking cones}

It was shown in Refs. [19, 29] that the locking behavior of different self-pulsating laser types can be described with the theory for injection locked electrical oscillators. Hence, the locking behavior of the AFL is given by the Adler [1] equation

$$
\begin{aligned}
\frac{d \phi(t)}{d t}+\left(\omega_{1}-\omega_{o}\right) & =-\Omega_{A} \sin \left[\phi(t)-\phi_{1}(t)\right], \\
\Omega_{A} & =\frac{\omega_{0}}{Q} \frac{P_{1}}{P_{0}} .
\end{aligned}
$$

Here, $\phi$ and $\phi_{1}$ denote the phase of the locked oscillator and the injected signal; $\omega_{0}$ and $\omega_{1}$ are the frequencies of the free running oscillator and the injected signal, respectively. $P_{0}$ and $P_{1}$ give the power of both signals and $Q$ describes the quality factor of the free running oscillator, i.e. the ratio of center frequency and the oscillator linewidth. Typical values of $10 \mathrm{MHz}$ linewidth and $40 \mathrm{GHz}$ center frequency correspond to $Q=$ 4000. From Eq. (9.1) it can be deduced that the locking range $\omega_{\text {lock }}$, where the AFL frequency adjusts to the injected signal, varies with the injected power as $\Delta \omega_{\text {lock }}=2 \Omega_{A}$. Therefore, the locking range increases linearly with the injected optical power [29], resulting in characteristic cones in the plane of frequency vs injected power. The linear increase with the signal power is due to the locking of the optical power instead of the electrical fields in electronic oscillators.
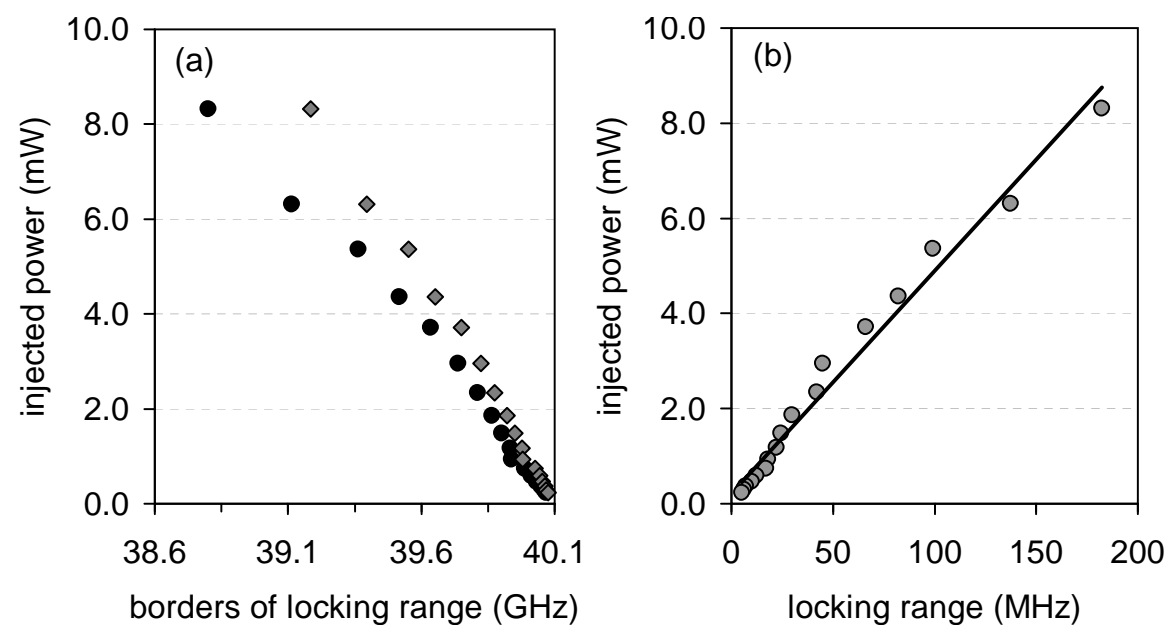

Figure 9.2: Locking cone of $40 \mathrm{GHz}$ MB pulsation. (a) Borders of locking range; (b) width of locking range. 


\section{Locking of AFL dynamics}

An example for a locking cone is given in Fig. 9.2. Here, a sinusoidal optical signal with a wavelength of $1550 \mathrm{~nm}$, adjustable power and a tunable frequency in the range of $40 \mathrm{GHz}$ were employed. The measurements verify that the locking range increases linearly with the injected power. An upper limit for the locking power is given by the shift of the laser operation point out of the stability range of the MB pulsations. ${ }^{3}$ The observed locking cone is equivalent to an Arnold tongue for a 1:1 locking ratio. Besides the linear increase of the locking range, an additional shift of the locking region center occurs. This is an effect induced by the gain suppression in the amplifier section due to the incoming data signal. The corresponding decrease in feedback strength lowers the MB frequency. From the application point of view, this limits the power dynamic range where the self-pulsations can be synchronized without readjusting the operation currents. Similar observations have been made for other types of self-pulsating lasers in Ref. [88].

Several experiments using the AFL $[18,20,21]$ for optical data processing have proven that this laser type is a promising candidate for all-optical clock recovery in data network applications.

\subsection{Superharmonic locking of PD}

The locking investigations in the previous Section have verified that the synchronization behavior obeys the laws for injection locked oscillators. The synchronization results from the power spectrum component having similar frequency as the fundamental frequency of the free running oscillator. In the typical synchronization scenario, a locking signal with frequencies below (subharmonic) or equal to the free running oscillator is used. In the case of a subharmonic data signal, the locking process mainly relies on the harmonics of the signal. Harmonics are generated due to nonlinearities, either during the signal generation, the transmission over a fiber, or when injecting sufficiently strong signals into the laser. These nonlinearities, however, do not generate subharmonics. Accordingly, the locking to a superharmonic signal $\left(f_{\text {lock }}=\mathrm{n} \cdot f_{S P}, \mathrm{n} \in \mathrm{N}\right)$ could not be observed for the AFL. On the other hand, the investigation of the resonances showed that $\mathrm{MB}$ and $\mathrm{RO}$ oscillations strongly interact, leading to mutual synchronization. This gives rise to the question if the internal synchronization mechanisms can be employed for superharmonic locking of RO pulsations.

For clarification, the PD scenario was investigated regarding its locking behavior to external signals. An operation point well within the range where both internal oscillators mutually synchronize was adjusted $\left(I_{A}=34.5 \mathrm{~mA}\right.$, for other device parameters see Section 7.1). A sinusoidal optical signal with a frequency of $24.6 \mathrm{GHz}$, an average power of $3 \mathrm{~mW}$ and a wavelength of $1550 \mathrm{~nm}$ was injected into the AFL as master signal. The suppression of a residual $12.3 \mathrm{GHz}$ component was larger than $30 \mathrm{~dB}$, hence excluding a direct synchronization of the ROs in the PD state. Reflections of the locking signal at the AFL facet are suppressed with an optical filter (3 $\mathrm{nm}$ bandwidth). Therefore,

\footnotetext{
${ }^{3}$ Another practical limitation is given by the facet damage threshold. It is reached at $30 \mathrm{~mW}$ optical injection power.
} 


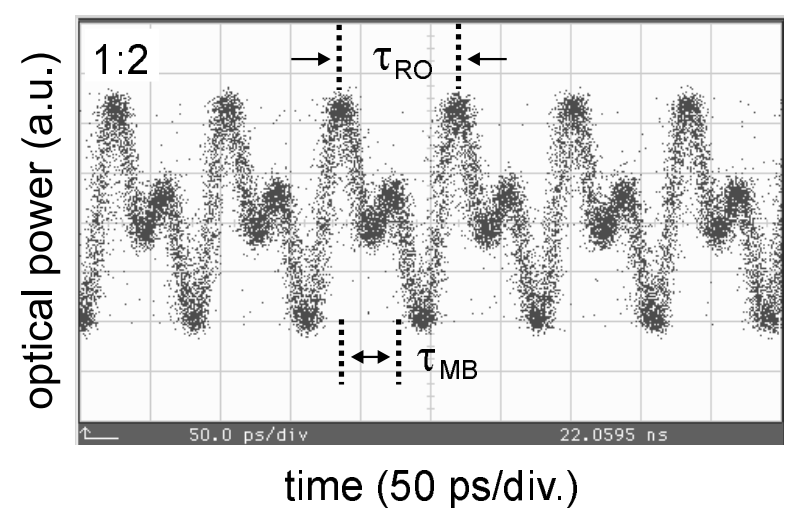

Figure 9.3: Locking of 1:2 scenario to optical master signals with a frequency equal to $f_{M B}$. The subharmonic RO oscillation is synchronized due to the internal oscillator interaction.

only the AFL dynamics is monitored by the sampling oscilloscope. Figure 9.3 shows the synchronized device output. One distinct pulse train is a clear indication that both oscillators are simultaneously locked to the master signal. The periodicity of the envelope corresponds to the $\mathrm{RO}$ frequency, with an additional amplitude modulation by the MB. The MB is directly locked by the injected signal frequency. Due to the internal locking mechanisms the RO pulsation also becomes synchronized and a clear pulse train is measured by the oscilloscope.

The locking behavior was also investigated for other resonant tori. For these experiments, the frequency of the master signal was adjusted with respect to the MB frequency, the other parameters of the sinusoidal signal remained unchanged. Compared to the 1:2 scenario, the synchronization for higher-order resonances becomes noisier. Nevertheless, the periodicity of a locked RO pulsation component is clearly visible. This result is in full agreement to the observations for the free running linewidths in the resonances (Fig. 7.2), where noise and the decreasing coupling for high frequency ratios led to a noise floor of the spectral components.

Thus, the AFL allows for functionalities beyond the all-optical extraction of the clock signal. Utilizing the internal synchronization mechanisms, all-optical frequency division becomes feasible. In addition to the importance for technical applications, the investigations in this Chapter show that the interaction of internal dynamics with external perturbations opens a new field of delayed feedback dynamics research. 


\section{Chapter 10}

\section{Summary and Outlook}

The present work demonstrates that lasers with monolithically integrated feedback cavities in the non-zero short delay limit provide complex dynamics. For the investigations a novel device type has been developed: the active feedback laser (AFL). This laser type allows the controlled preparation of the characteristic dynamics in the very short feedback cavity regime.

First, the occurrence of non-trivial dynamics in lasers with very short feedback delay was shown with a passive feedback laser (PFL). The PFL allowed the experimental identification of the generic pulsations types: undamped relaxation oscillations (RO) and beating of a pair of longitudinal modes (mode beating, MB). However, it turned out that adjusting the dynamics in the PFL is strongly limited by the lack of independent control mechanisms for feedback phase and strength.

As a consequence, the AFL was developed. The combination of separate phase and amplifier sections enables the adjustment of feedback phase and strength. The amplification in the feedback section can compensate for optical losses and the whole range of feedback strengths becomes available. Therefore, the dynamics in the very short feedback cavity regime could be investigated employing a single device.

Characteristic instabilities of single-mode states are undamped ROs. They originate from the intrinsic dispersion of the feedback section and have frequencies slightly enhanced with respect to the solitary relaxation oscillations. At higher feedback strength, MB phenomena are observed due to the coexistence of longitudinal compound-cavity modes. The frequency of the beating pulsations depends on the feedback strength and can be tuned in a wide range.

Phenomena typical for nonlinear systems organize the regions of RO and MB pulsations. Hysteresis occurs due to the coexistence of several laser states. The transition from stationary lasing to RO and $\mathrm{MB}$ pulsations are induced by supercritical Hopf bifurcations. RO pulsations loose their stability either by Hopf or homoclinic bifurcations returning the laser into a stable continuous wave emission. Period doubling and torus bifurcations represent the borders of the beating pulsation areas. The physical mechanism underlying the torus scenario is the coexistence of MB pulsations and ROs of the compound device. In the torus regime, several phenomena characteristic for coexisting oscillators were observed. Resonances occur for rational frequency ratios. 
They can be identified by a decoupling of the dynamics from the control parameter and a significant reduction in linewidth. Additionally, the break-up of the torus towards the island border could be demonstrated. A boundary crisis scenario was identified by simulations.

Modifications of the intrinsic dynamics can arise from internal or external perturbations. Noisy precursors and irregular dynamics at the transition from RO to $\mathrm{MB}$ are the result of spontaneous emission noise. The synchronization to periodic external signals was verified. It is the prerequisite for the application of AFLs for all-optical clock recovery or frequency division.

In summary, the most important discovery of this thesis is that active feedback makes the short feedback cavity regime accessible for experiments. Thereby, the active feedback laser can serve as miniature laboratory for the verification of nonlinear dynamics. A step towards the thorough understanding of phenomena in delayed feedback lasers was made.

Although the present work has given a comprehensive overview on the characteristic dynamics of the AFL, several issues are promising candidates for further investigations.

Aiming for a technical application of the AFL, e.g. in communication networks, the device dynamics has to be tailored with respect to the particular demands. Key issues are the extension of the pulsation frequency range and a minimization of the timing jitter. The transfer of the AFL concept to vertical cavity structures may be attractive, since it allows a simple coupling to optical fibers. Chaotic dynamics in the AFL makes it suitable for the application as a robust and compact source for secure communication.

Apart from the technical use, the AFL can also serve for fundamental studies of nonlinear dynamics. Simulations and experiments have shown that the location of bifurcations sensitively depends on the device parameters. Controlling the location of the bifurcations with the device parameters would allow for a direct 'design' of dynamical scenarios. Potentially interesting are situations, where hysteresis disappears due to the coincidence of bifurcations. Here, distinct phenomena can be expected. One example is excitability at the homoclinic border of the RO island. Additionally, coherence resonance phenomena can arise when a torus border coincides with a saddlenode bifurcation. In such scenarios, external noise is expected to increase the regularity of the laser emission. 



\section{Appendix A}

\section{Appendix}

\section{A.1 Device parameter used for simulation}

\begin{tabular}{|c|l|c|c|c|l|}
\hline & explanation & \multicolumn{3}{|c|}{ values } & unit \\
\hline$\kappa$ & index coupling coefficient & 130 & 0 & 0 & $\mathrm{~cm}^{-1}$ \\
$l$ & section length & 200 & 350 & 250 & $\mu \mathrm{m}$ \\
$\sigma$ & cross-section of AZ & 0.45 & & 0.45 & $\mu \mathrm{m}^{2}$ \\
$g^{\prime}$ & effective differential gain & 9 & & 9 & $10^{-17} \mathrm{~cm}^{2}$ \\
$\alpha_{H}$ & Henry factor & -5 & & -5 & \\
$\alpha$ & internal absorption & 25 & {$[20,40]$} & 25 & $\mathrm{~cm}^{-1}$ \\
$\delta$ & static detuning & 402.7 & & {$[0,160]$} & $\mathrm{cm}^{-1}$ \\
$I$ & current injection & 70 & & {$[0,100]$} & $\mathrm{mA}^{18}$ \\
$n_{t r}$ & transparency carrier density & 1 & & 1 & $10^{18} \mathrm{~cm}^{-3}$ \\
$A$ & recombination coefficient & 0.3 & & 0.3 & $10^{9} \mathrm{~s}^{-1}$ \\
$B$ & recombination coefficient & 1 & & 1 & $10^{-10} \mathrm{~cm}^{3} \mathrm{~s}^{-1}$ \\
$C$ & recombination coefficient & 1 & & 1 & $10^{-28} \mathrm{~cm}^{6} \mathrm{~s}^{-1}$ \\
$\varepsilon$ & nonlinear gain compression & 3 & & 3 & $10^{-18} \mathrm{~cm}^{3}$ \\
$U_{F}^{\prime}$ & diff. Fermi level separation & 1 & & 1 & $10^{-19} \mathrm{~V}^{3} \mathrm{~cm}^{3}$ \\
$R_{s}$ & series resistivity & 5 & & 5 & $\Omega$ \\
$\bar{g}$ & Lorentzian height & 200 & 0 & 200 & $\mathrm{~cm}^{-1}$ \\
$\bar{\gamma}$ & Lorentzian half width & 23.84 & & 23.84 & $\mathrm{rad} / \mathrm{ps}^{-1}$ \\
$\bar{\omega}$ & Lorentzian central frequency & 2.384 & & 2.384 & $\mathrm{rad} / \mathrm{ps}^{-3}$ \\
$\lambda_{0}$ & central wavelength & 1540 & & & $\mathrm{~nm}$ \\
$R$ & power reflectivity & & & 0.3 & \\
$v_{g}$ & group velocity & & $c / 3.8$ & & \\
\hline
\end{tabular}

Table A.1: Parameter values used for the DFB, phase tuning (P), and amplifier (A) sections. Deviations are noted in the text. 


\section{A.2 List of Abbreviations}

\begin{tabular}{|c|c|}
\hline AFL & active feedback laser \\
\hline AR & anti-reflection \\
\hline ASE & amplified spontaneous emission \\
\hline $\mathrm{CC}$ & coherence collapse \\
\hline CSA & communication signal analyzer (fast sampling oscilloscope) \\
\hline $\mathbf{c w}$ & continuous wave \\
\hline dc & direct current \\
\hline DFB & distributed feedback \\
\hline DQS & dispersive Q-switching \\
\hline $\mathrm{EC}(\mathrm{M})$ & external cavity (mode) \\
\hline ECL & external cavity laser \\
\hline EDFA & erbium doped fiber amplifier \\
\hline ESA & electrical spectrum analyzer \\
\hline FP & Fabry-Perot \\
\hline FWHM & full-width at half maximum \\
\hline LCR & long cavity regime \\
\hline LFF & low frequency fluctuations \\
\hline LK & Lang-Kobayashi \\
\hline LSHB & longitudinal spatial hole burning \\
\hline MB & mode beating \\
\hline $\operatorname{ML}(\mathrm{L})$ & mode locked (laser) \\
\hline OSA & optical spectrum analyzer \\
\hline PD & period doubling \\
\hline PFL & passive feedback laser \\
\hline RBw & resolution bandwidth \\
\hline rf & radio frequency \\
\hline RIN & relative intensity noise \\
\hline rms & root mean square \\
\hline $\mathrm{RO}$ & relaxation oscillation \\
\hline SCR & short cavity regime \\
\hline SL & semiconductor laser \\
\hline $\mathrm{SN}(\mathrm{OLC})$ & saddle-node (of limit cycle) \\
\hline SP & self-pulsation \\
\hline SPL & self-pulsating laser \\
\hline TE & transversal electric \\
\hline $\mathrm{TM}$ & transversal magnetic \\
\hline TWE & traveling-wave equation \\
\hline
\end{tabular}




\section{A.3 Modifications of TWE-model}

A brief description of the theory for DFB lasers is presented in Chapter 2. In reality, several effects modify the basic behavior. The TWEs can be expanded to account for effects like spontaneous emission noise, gain dispersion, nonlinear gain, and longitudinal spatial hole burning (LSHB) [97].

\section{Modifications of optical equations}

The optical field in a laser is described by two coupled-wave equations [84]

$$
\begin{aligned}
& \left(-i v_{g}^{-1} \partial_{t}-i \partial_{z}+\beta\right) E^{+}+\kappa^{+} E^{-}=F_{s p}^{+} \\
& \left(-i v_{g}^{-1} \partial_{t}+i \partial_{z}+\beta\right) E^{-}+\kappa^{-} E^{+}=F_{s p}^{-}
\end{aligned}
$$

Variables in these equations are chosen according to Chapter 2. In order to take into account spontaneous emission noise, the TWEs are supplemented by Langevin noise sources $F_{s p}^{ \pm}$. Gain dispersion and nonlinear gain suppression are introduced by a modified propagation parameter

$$
\beta=\left(i+\alpha_{H}\right) \frac{g}{2}-i \frac{\alpha_{0}}{2}+\delta-i D
$$

The real constant $\delta$ gives the background wavenumber relative to the Bragg-resonance. Nonlinear gain-suppression modifies the gain model as

$$
g\left(n, \varepsilon|E|^{2}\right)=\frac{g^{\prime}\left(n-n_{t r}\right)}{1+\varepsilon|E|^{2}} .
$$

$n$ and $n_{t r}$ denote the carrier density and the density at transparency, respectively. By $\varepsilon$ the gain becomes dependent on the optical intensity and saturates for high values. The operator $D$ in the propagation constant incorporates the gain dispersion, which can have a strong impact on the mode selection in the laser. $D$ is defined as

$$
D E^{ \pm}=\frac{\bar{g}}{2}\left(E^{ \pm}-p^{ \pm}\right)
$$

and for the polarization $p^{ \pm}$a single-oscillator model is used:

$$
-i \partial_{t} p^{ \pm}=-i \bar{\gamma}\left(E^{ \pm}-p^{ \pm}\right)+\bar{\omega} p^{ \pm}
$$

where $\bar{\omega}$ is the resonance frequency taken relative to the central frequency $2 \pi c / \lambda_{0}, g-\bar{g}$ the off-resonance gain, and $\bar{\gamma}$ measures the gain bandwidth [7].

\section{Inhomogeneous carrier distribution due to LSHB}

When LSHB is taken into account the carrier density equation has to be modified. A continuous spatial variation of the carrier density $n$ is regarded by the equation

$$
\partial_{t} n=j-R(n)-\frac{c}{n_{g}} \operatorname{Re}\left[E^{*}(g+2 D) E\right] .
$$




\section{A. Appendix}

$R(n)=A n+B n^{2}+C n^{3}$ accounts for the spontaneous recombination. The stimulated recombination term is not averaged over the section, hence, the density can vary with the longitudinal position $z$. In the stimulated recombination term neither carrier diffusion nor forward-backward wave interferences (e.g. $\sim E^{+} \beta E^{-}$) appear. The spatial period of the latter is shorter than the carrier diffusion length by more than an order of magnitude and thus not transferred to the carrier density. On the other hand, after having dropped these mixed terms, the stimulated recombination varies on a scale much longer than the diffusion length, with the consequence that diffusion can be dropped, too. Due to the variation of stimulated recombination along the cavity, the injection rate $j$ in Eq. (A.6) also becomes inhomogeneous, ensuring a constant voltage along the laser contact. This is regarded by $[6,24,33,34]$

$$
j(z, t)=\frac{I}{e V}-\frac{U_{F}^{\prime}}{e V R_{s}}(n(z, t)-\bar{n}(t)) .
$$

The first term describes the average injection rate which is modified by the effects of carrier self-distribution (second term). The differential Fermi-level separation $U_{F}^{\prime}$ and the series resistivity $R_{s}$ are treated as constant parameters, $n(z, t)$ is the time dependent local carrier density, and $\bar{n}$ denotes the average density in the laser section. The transverse confinement factor $\Gamma$ is already incorporated in $g^{\prime}, \varepsilon$, and $\bar{g}$. For multisection lasers the characteristics of the different sections (phase/amplifier section) and appropriate boundary conditions have to be taken into account. 


\section{A.4 Stability of AFL dynamics}

A single AFL is investigated throughout this work. Additionally, for most investigations the DFB injection remains fixed at $70 \mathrm{~mA}$ to simplify measurements. This raises the question, whether the investigations are also valid for varied device geometry and how the DFB injection influences the dynamics.

In order to show that moderate variations of device geometry (i.e. remaining in the very short cavity regime) have no major impact on the general AFL behavior, a modified device has been realized. The laser was cleaved from another wafer than previously investigated devices. Hetero-structure and the basic device layout remained unchanged. Deviating parameters are a lasing wavelength of $1550 \mathrm{~nm}$ and a coupling coefficient of $\kappa=140 \mathrm{~cm}^{-1}$. The section lengths are $210 \mu \mathrm{m}, 550 \mu \mathrm{m}$, and $250 \mu \mathrm{m}$ for laser, phase and amplifier section, respectively. By the longer feedback cavity, the spacing of ECMs becomes smaller and mode beating (MB) frequencies are lower. A detailed investigation of the relation between upper frequency limit and feedback cavity length is given in [9].

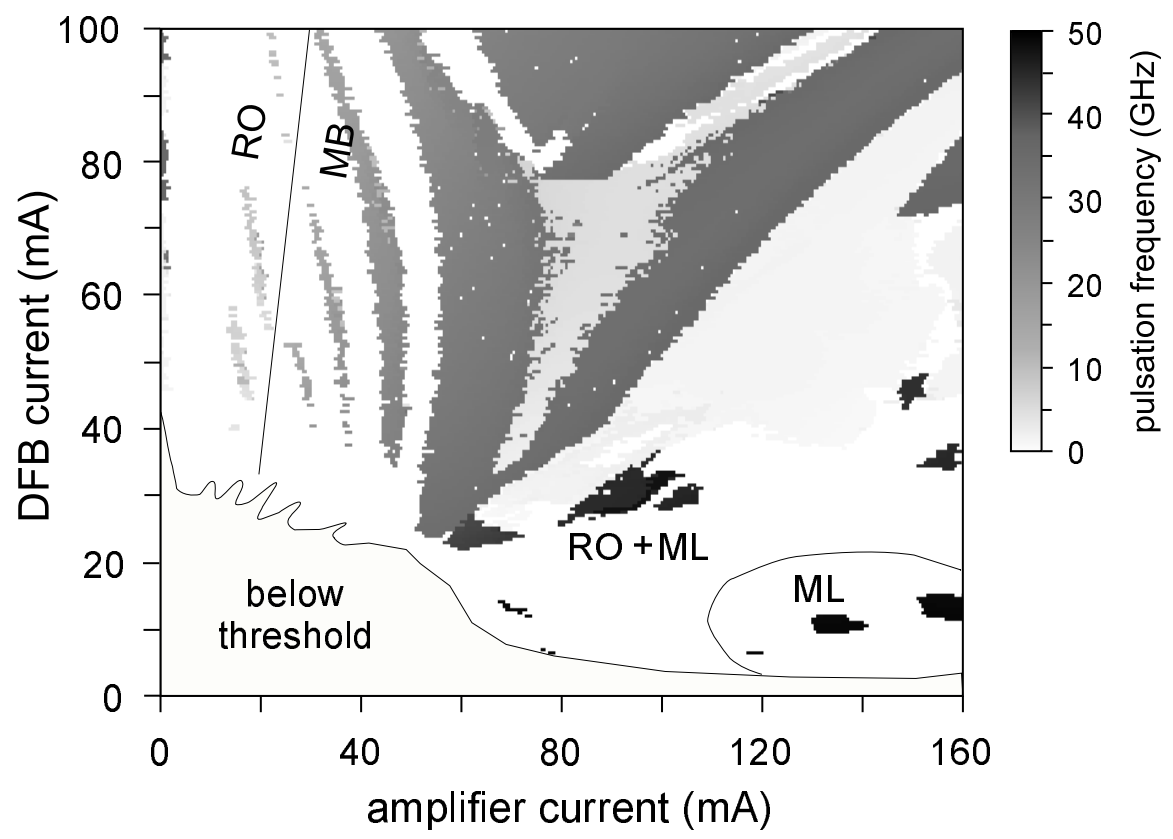

Figure A.1: Overview measurement in the plane of $I_{A}$ and $I_{D F B} . \mathrm{RO}$ and MB pulsations are observed. For low injection into the DFB section passive mode-locking (ML) occurs (indicated pulsations exceed the noise level by more than $20 \mathrm{~dB}$ ). Current combinations where the laser is below threshold are indicated.

Figure A.1 summarizes the characteristics of the modified device. The measurement was performed as described in Section 4.4.2, here with $I_{D F B}$ and $I_{A}$ as parameters, $I_{P}$ was fixed to $6 \mathrm{~mA}$. This allows to asses the impact of the DFB bias on the device dynamics. The lasing threshold of the compound-cavity is indicated, illustrating the effect of two gain sections in the laser cavity: the threshold becomes strongly dependent 


\section{A. Appendix}

on the distribution of the total injected current within the active sections. Changing $I_{P}$ shifts the threshold within a few $\mathrm{mA}$.

Above threshold, mainly the expected dynamical regimes can be identified. At low amplifier currents $\left(I_{A}<20 \mathrm{~mA}\right)$ undamped relaxation oscillations (ROs) occur. Their frequency increases with the injected DFB current. Higher feedback strengths result in the typical MB pulsations, until above $80 \mathrm{~mA}$ the torus dynamics and chaotic emission dominates. The onset of the irregular regime strongly depends on the DFB current. An increased injection into the DFB section effects an increased photon density. As a result, gain suppression in the amplifier section is induced, which has to be counteracted by higher $I_{A}$.

For low $I_{D F B}$ and strong injection into the amplifier section an additional operation regime occurs. Here, the DFB section looses its role of defining a single mode as organizing center for the AFL dynamics. It mainly acts as a mirror to the feedback cavity, which becomes the lasing part of the AFL. In this operation regime several longitudinal modes are coupled, giving rise to a passively mode-locked operation [3]. The observed pulsation frequency is $48.7 \mathrm{GHz}$, indicating an effective cavity length defined by phase and amplifier plus a penetration depth into the DFB grating of $60 \mu \mathrm{m}$. The stable synchronization of the locked modes results in a FWHM of the rf spectrum of $3 \mathrm{MHz}$. Differences between the mode-locked state and MB pulsations become clear when the injection values are modified: a change of operation currents allows only a frequency variation of $100 \mathrm{MHz}$ before the mode-locked state breaks down. It has been demonstrated in Ref. [13] that the pulses in mode-locked operation have a width of $\sim 6 \mathrm{ps}$, i.e. considerably shorter than MB pulses with the same frequency. Towards higher injection into the DFB section the mode-locked state becomes unstable and concurrent ROs occur.

In summary, neglecting the DFB current as parameter is reasonable, as long as the DFB section defines a single lasing mode. The main impact of a cavity length variation is a change of the upper limit for pulsation frequencies. 


\section{Bibliography}

[1] R. Adler. A study of locking phenomena in oscillators. Proc. IRE, 34:1380-1385, June 1946.

[2] P. Andrekson, N. Olsson, T. Tanbun-Ek, R. Logan, D. Coblentz, and H. Temkin. Novel technique for determining optical loss of individual semiconductor laser. Electron. Lett., 28:171-172, 1992.

[3] E. A. Avrutin, J. Marsh, and E. Portnoi. Monolithic and multi-GigaHertz modelocked semiconductor lasers: Constructions, experiments, models and applications. IEE Proc.-Optoelectron., 147(4):251-278, 2000.

[4] U. Bandelow. Theorie longitudinaler Effekte in 1.55 $\mu \mathrm{m}$ Mehrsektions DFB Laserdioden. PhD thesis, Humboldt-Universität zu Berlin, 1994.

[5] U. Bandelow, R. Schatz, and H.-J. Wünsche. A correct single-mode photon rate equation for multi-section lasers. IEEE Photon. Technol. Lett., 8(5):614-616, 1996.

[6] U. Bandelow, H. Wenzel, and H.-J. Wünsche. Influence of inhomogeneous injection on sidemode suppression in strongly coupled DFB semiconductor lasers. Electron. Lett., 28(14):1324-1325, 1992.

[7] U. Bandelow, M. Wolfrum, M. Radziunas, and J. Sieber. Impact of Gain Dispersion on the Spatio-temporal Dynamics of Multisection Lasers. IEEE J. Quantum Electron., 37(2):183-189, 2001.

[8] U. Bandelow, H.-J. Wünsche, B. Sartorius, and M. Möhrle. Dispersive self Qswitching in DFB-lasers: Theory versus experiment. IEEE J. Sel. Topics in Quantum Electron., 3:270-278, 1997.

[9] S. Bauer, O. Brox, M. Biletzke, J. Kreissl, M. Radziunas, B. Sartorius, and H.-J. Wünsche. Speed Potential of Active Feedback Lasers. In Proceedings of CLEOe 2003, page CC1M, 2003.

[10] S. Bauer, O. Brox, C. Bornholdt, J. Slovak, J. Kreissl, and B. Sartorius. SelfPulsating Multi-Section DFB Lasers and Their Applications. In Proceedings of IEEE LEOS 2003 Annual Meeting, Tucson, Arizona, volume 1, pages 45-46, 2003. 
[11] S. Bauer, O. Brox, J. Kreissl, G. Sahin, and B. Sartorius. Optical Microwave Source. Electron. Lett., 38:334-335, 2002.

[12] S. Bauer, O. Brox, J. Kreissl, B. Sartorius, M. Radziunas, J. Sieber, H.-J. Wünsche, and F. Henneberger. Nonlinear dynamics of semiconductor lasers with active optical feedback. Phys. Rev. E, 69:016206, 2004.

[13] S. Bauer, B. Hüttl, O. Brox, M. Biletzke, J. Kreissl, B. Sartorius, and H.-J. Wünsche. Unifying mode-locking and self-pulsations in a laser with active feedback. Proceedings of the OAA2004, page OTuD2, 2004.

[14] B. R. Bennet, R. A. Soref, and J. A. del Alamo. Carrier-Induced Change in Refractive Index of InP, GaAs, and InGaAsP. IEEE J. Quantum Electron., 26(1):113$122,1990$.

[15] A. Betke. Erregbarkeit in Mehrsektions-Halbleiterlasern. Master's thesis, Humboldt-Universität zu Berlin, 2003.

[16] J. R. Biard, W. N. Carr, and B. S. Reed. Analysis of a GaAs Laser. Trans. Metallurg. Soc. AIME, 230:286-290, 1964.

[17] O. Brox. Optische Signalverarbeitung mit DFB-Laser mit kurzer optischer Rückkopplung. PhD thesis, TU Berlin, 2004.

[18] O. Brox, S. Bauer, M. Biletzke, H. Ding, J. Kreissl, H.-J. Wünsche, and B. Sartorius. Short Feedback DFB for $40 \mathrm{GHz}$ Clock Recovery: Noise Induced Intensity Fluctuations and Jitter. In Proceedings of the OFC2004, Los Angeles, California, page MF55, 2004.

[19] O. Brox, S. Bauer, C. Bobbert, M. Biletzke, J. Kreissl, H.-J. Wünsche, and B. Sartorius. Timing Stability of Injection-Locked $40 \mathrm{GHz}$ Self-Pulsating DFB-Lasers. In Proceedings of the ECOC2003, Rimini, Italy, page Tu4.5.3, 2003.

[20] O. Brox, S. Bauer, C. Bobbert, G. Bramann, J. Kreissl, B. Sartorius, M. Schmidt, K. Schuh, B. Junginger, and E. Lach. 160 to 40 Gb/s Demultiplexing Using a SelfPulsating Laser Based Clock Recovery. In Proceedings of the OFC2003, Georgia, Atlanta, volume 1, pages 105-106, 2003.

[21] O. Brox, S. Bauer, J. Kreissl, M. Möhrle, B. Sartorius, and H.-J. Wünsche. DFBLasers with Amplified Optical Feedback for Tunable High-Frequency Generation. In Trends in Optics and Photonics Series - Optical Amplifier and Their Applications, volume 77, page OWC2, 2002.

[22] O. Brox, S. Bauer, M. Radziunas, M. Wolfrum, J. Sieber, J. Kreissl, B. Sartorius, and H.-J. Wünsche. Tunable High-Frequency Generation in DFB-Lasers with Amplified Feedback. IEEE J. Quantum Electron., 39(11):1381-87, 2003. 
[23] J. Carroll, J. Whiteaway, and D. Plumb. Distributed Feedback Semiconductor Lasers. IEE, London, 1998.

[24] Y. Champagne and N. McCarthy. Influence of axially varying quasi-Fermi-level separation of the active region on spatial hole burning in distributed feedback lasers. J. Appl. Phys., 72:2110-2118, 1992.

[25] L. C. Coldren and S. W. Corzine. Diode Lasers and Photonic integrated Circuits. John Wiley and Sons, New York, 1995.

[26] P. Correc. Stability of phase-shifted DFB lasers against hole burning. IEEE J. Quantum Electron., 30(11):2467-2476, 1994.

[27] M. W. Derstine, H. M. Gibbs, F. A. Hopf, and D. L. Kaplan. Bifurcation gap in a hybrid optically bistable system. Phys. Rev. A, 26(6):3720-3722, 1982.

[28] E. J. Doedel, A. R. Champneys, T. F. Fairgrieve, Y. A. Kuznetsov, B. Sandstede, and X. Wang. AUTO97, Continuation and bifurcation software for ordinary differential equations, 1998.

[29] G.-H. Duan and G. Pham. Injection locking properties of self pulsation in semiconductor lasers. IEE Proc. Optoelectr., 144(4):228-234, 1997.

[30] A. A. Duarte and H. G. Solari. Stability properties of the monochromatic spectrum in a double cavity laser. Phys. Rev. A., 60:2403, 1999.

[31] J. Dubbeldam and B. Krauskopf. Self-pulsation of lasers with saturable absorber: dynamics and bifurcations. Opt. Comm., 159:325-338, 1999.

[32] K. J. Ebeling. Integrierte Optoelektronik. Springer Verlag, Berlin, 1989.

[33] P. G. Eliseev and A. E. Drakin. Self-distribution of the current in laser diodes and its possible use for reducing the nonlinearity of the active medium. IEEE $J$. Quantum Electron., 26:299-302, 1996.

[34] P. G. Eliseev, A. G. Glebov, and M. Osinski. Current Self-Distribution Effect in Diode Lasers: Analytic Criterion and Numerical Study. IEEE J. on Sel. Top. in Quantum Electron., 3(2):499-506, 1997.

[35] K. Engelborghs, T. Luzyanina, and D. Roose. Numerical bifurcation analysis of delay differential equations using DDE-BIFTOOL. ACM Trans. on Math. Software, 28(1):1-21, 2002.

[36] T. Erneux, F. Rogister, A. Gavrielides, and V. Kovanis. Bifurcation to mixed external cavity mode solutions for semiconductor lasers subject to external feedback. Opt. Comm., 183:467-477, 2000. 
[37] I. Fischer, G. H. M. Tartwijk, A. M. Levine, W. Elsäßer, E. O. Göbel, and D. Lenstra. Fast Pulsing and Chaotic Itinerancy with a Drift in the Coherence Collapse of Semiconductor Lasers. Phys. Rev. Lett., 76:220, 1996.

[38] T. Fukushima, R. Nagarajan, M. Ishikawa, and J. E. Bowers. High-Speed Dynamics in InP Based Multiple Quantum Well Lasers. Jpn. J. Appl. Phys. Pt. I, 32(1A):70-83, 1993.

[39] C. Grebogi, E. Ott, and J. A. Yorke. Chaotic Attractors in Crisis. Phys. Rev. Lett., 48(22):1507-10, 1982.

[40] J. Guckenheimer and P. Holmes. Nonlinear Oscillations, Dynamical Systems, and Bifurcations of Vector Fields. Springer Verlag, Berlin, 1983.

[41] H. Haken. Synergetics. Springer-Verlag, Berlin, 1983.

[42] B. W. Hakki and T. L. Paoli. Gain spectra in GaAs double hetero-junction injection lasers. J. Appl. Phys., 46:1299-1306, 1975.

[43] R. N. Hall, G. E. Fenner, J. D. Kingsley, T. J. Soltys, and R. O. Carlson. Coherent Light Emission From GaAs Junctions. Phys. Rev. Lett., 9(9):366-368, 1962.

[44] G. Harris. Worldwide market 2005. Opto Ef Laser Europe, April 2002.

[45] T. Heil, I. Fischer, W. Elsäßer, and A. Gavrielides. Dynamics of semiconductor lasers subject to delayed optical feedback: The short cavity regime. Phys. Rev. Lett., 87:243901, 2001.

[46] T. Heil, I. Fischer, W. Elsäßer, B. Krauskopf, K. Green, and A. Gavrielides. Delay dynamics of semiconductor lasers with short external cavities: Bifurcation scenarios and mechanisms. Phys. Rev. E, 67:066214, 2003.

[47] I. D. Henning and J. V. Collins. Measurements of the Semiconductor Linewidth Broadening Factor. Electron. Lett., 19(22):927-929, 1983.

[48] W. B. Joyce and R. W. Dixon. Analytic Approximation of the Fermi Energy of an Ideal Fermi Gas. Appl. Phys. Letters, 31(5):354-56, 1977.

[49] Y. H. Kao and H. T. Lin. Persistent properties of period doubling in directly modulated semiconductor lasers. Phys. Rev. A, 48(3):2292-2298, 1993.

[50] S.-B. Kim and J.-S. Cho. Sampled Grating Conventional DFB Lasers with Fabricability and High-Immunity to the Spatial-Hole Burning. IEEE Photon. Technol. Lett., 9(5):560-562, 1997.

[51] O. Kjebon, R. Schatz, S. Lourdudoss, S. Nilsson, and B. Stalnacke. Modulation response measurements and evaluation of MQW InGaAsP laser of various designs. Proc. SPIE, 2684:138-152, 1996. 
[52] H. Kogelnik and C. V. Shank. Coupled-Wave Theory of Distributed Feedback Lasers. J. Appl. Phys., 43:2325-2335, 1972.

[53] K. Komori, S. Arai, Y. Suematsu, I. Arima, and M. Aoki. Single-Mode Properties of Distributed-Reflector Lasers. IEEE J. Quantum Electron., 25(6):1235-1244, 1989.

[54] N. Korneyev, M. Radziunas, H.-J. Wünsche, and F. Henneberger. Bifurcations of a DFB Laser with Short Optical Feedback: Numerical Experiment. Proceedings of SPIE, 4986(9):480-489, 2003.

[55] B. Krauskopf. Bifurcation analysis of laser systems. In B. Krauskopf and D. Lenstra, editors, AIP Conf. Proc., volume 548, page 1. AIP Melville, New York, 2000.

[56] M. Kuznetsov. Theory of Wavelength Tuning in Two-Segment Distributed Feedback Lasers. IEEE J. Quantum Electron., 24(9):1837-1844, 1988.

[57] Y. Kuznetsov. Elements of Applied Bifurcation Theory. Springer Verlag, Berlin, 1995.

[58] R. Lang and K. Kobayashi. External optical feedback effects on semiconductor injection properties. IEEE J. Quantum Electron., 16:347-355, 1980.

[59] C. H. Lee, T. H. Yoon, and S. Y. Shin. Period doubling and chaos in a directly modulated laser diode. Appl. Phys. Lett., 46(1):95-97, 1990.

[60] A. J. Lowery. Dynamics of SHB-induced mode-instabilities in uniform DFB semiconductor laser. Electron. Lett., 29:1852-1853, 1993.

[61] P. Mandel, C. Etrich, and K. Otsuka. Laser rate equations with phase-sensitive interactions. IEEE J. Quantum Electron., 29:836-843, 1993.

[62] C. Masoller and N. B. Abraham. Stability and modulation properties of a semiconductor laser with weak optical feedback from a distant reflector. Quantum Semiclass. Opt., 10:519-534, 1998.

[63] M. Möhrle, U. Feiste, J. Hörer, R. Molt, and B. Sartorius. Gigahertz Self-Pulsation in $1.5 \mu \mathrm{m}$ Wavelength Multisection DFB Lasers. IEEE Photon. Technol. Lett., 4(9):976-978, 1992.

[64] M. Möhrle, B. Sartorius, C. Bornholdt, S. Bauer, O. Brox, A. Sigmund, R. Steingrüber, M. Radziunas, and H.-J. Wünsche. Detuned Grating Multisection-RWDFB Lasers for High Speed Optical Signal Processing. IEEE J. on Sel. Top. Quantum Electron., 7:217-223, 2001.

[65] J. Mørk, B. Tromborg, and J. Mark. Chaos in Semiconductor Lasers with Optical Feedback: Theory and Experiment. IEEE J. Quantum Electron., 28(1):93-108, 1992. 
[66] G. Morthier and P. Vankwikelberge. Handbook of Distributed Feedback Laser Diodes. Artech House, 1997.

[67] J. Ohtsubo. Chaos synchronisation and chaotic signal masking in semiconductor lasers with optical feedback. IEEE J. Quantum Electron., 38(9):1141-1154, 2002.

[68] M. Osinski and J. Buus. Linewidth broadening factor in semiconductor lasers An overview. IEEE J. Quantum Electron., 23:9-29, 1987.

[69] K. Petermann. Laser diode modulation and noise. Kluwer Academic Publishers, 1991.

[70] K. Petermann. External Optical Feedback Phenomena in Semiconductor Lasers. IEEE J. on Sel. Top. in Quantum Electron., 1(2):480-489, 1995.

[71] D. Pieroux, T. Erneux, B. Haegeman, K. Engelborghs, and D. Roose. Bridges of Periodic Solutions and Tori in Semiconductor Lasers Subject to Delay. Phys. Rev. Lett., 87(19):193901, 2001.

[72] D. Pieroux and P. Mandel. Low-frequency fluctuations in the Lang-Kobayashi equations. Phys. Rev. E, 68:036204, 2003.

[73] A. S. Pikovsky, M. G. Rosenblum, and J. Kurths. Synchronization, A Universal Concept in Nonlinear Sciences. Cambridge University Press, Cambridge, 2001.

[74] W. S. Rabinovich and B. J. Feldman. Spatial Hole Burning Effects in Distributed Feedback Lasers. IEEE J. Quantum Electron., 25(1):20-30, 1989.

[75] M. Radziunas and H.-J. Wünsche. Dynamics of multi-section DFB semiconductor laser: Traveling wave and mode approximation models. In Proceedings of SPIE, volume 4646, pages 27-37, 2002.

[76] J. Renaudier. Fast Characterisation Method for Multi-Section Semiconductor Lasers for Optical Signal Processing. Master's thesis, ENST De Bretagne - France, and HHI, Berlin, Germany, 2002.

[77] C. Robert, K. T. Alligood, E. Ott, and J. A. Yorke. Explosions of chaotic sets. Physica D, 144:44-61, 2000.

[78] T. Sano. Antimode dynamics and chaotic itinerancy in the coherence collapse of semiconductor lasers with optical feedback. Phys. Rev. A, 50(3):2719-2726, 1994.

[79] R. Schatz. Longitudinal Spatial Instability in Symmetric Semiconductor Lasers due to Spatial Hole Burning. IEEE J. Quantum Electron., 28(6):1443-1449, 1992.

[80] J. Sieber. Numerical bifurcation analysis for multi-section semiconductor lasers. SIAM J. of Appl. Dyn. Sys., 1(2):248-270, 2002. 
[81] J. Sieber, M. Radziunas, and K. R. Schneider. Dynamics of Multisection Semiconductor Lasers. Mathematical Modelling and Analysis, 9(1):51-66, 2004.

[82] A. A. Tager and K. Petermann. High-Frequency Oscillations and Self-Mode Locking in Short External-Cavity Laser Diodes. IEEE J. Quantum Electron., 30(7):1553-1561, 1994.

[83] R. W. Tkach and A. R. Chraplyvy. Regimes of feedback effects in 1.5- $\mu \mathrm{m}$ Distibuted Feedback Lasers. IEEE Journ. Lightwave Technol., LT-4(11):1655-1661, 1986.

[84] B. Tromborg, H. E. Lassen, and H. Olesen. Travelling Wave Analysis of Semiconductor Lasers. IEEE J. Quantum Electron., 30(5):939-956, 1994.

[85] V. Tronciu, H.-J. Wünsche, J. Sieber, K. Schneider, and F. Henneberger. Dynamics of single mode semiconductor lasers with passive dispersive reflectors. Opt. Comm., 182:221228, 2000.

[86] O. Ushakov, S. Bauer, O. Brox, H.-J. Wünsche, and F. Henneberger. SelfOrganization in Semiconductor Lasers with Ultrashort Optical Feedback. Phys. Rev. Lett., 92(4):043902, 2004.

[87] G. H. M. van Tartwijk and G. P. Agrawal. Laser instabilites: a modern perspective. Prog. in Quant. Electron., 22:43-122, 1998.

[88] A. Vizzino, M. Gioannini, and I. Montrosset. Dynamic simulation of clock recovery with self-pulsating three-section distributed-feedback lasers. IEEE J. Quantum Electron., 38(12):1580-1586, 2002.

[89] C. O. Weiss and R. Vilaseca. Dynamics of Lasers. Wiley, Weinheim, 1991.

[90] H. Wenzel. Green's Function Based Simulation of the Optical Spectrum of Multisection Lasers. IEEE J. Sel. Top. in Quantum Electron., 9(3):865-871, 2003.

[91] H. Wenzel, U. Bandelow, H.-J. Wünsche, and J. Rehberg. Mechanisms of fast self pulsations in two-section DFB lasers. IEEE J. Quantum Electron., 32(1):69-79, 1996.

[92] S. Wieczorek, B. Krauskopf, and D. Lenstra. A unifying view of bifurcations in a semiconductor laser subject to optical injection. Opt. Comm., 172:279-295, 1999.

[93] K. Wiesenfeld. Virtual Hopf Phenomenon: A new precursor of period doubling bifurcations. Phys. Rev. A, 32(3):1744-1751, 1985.

[94] M. Wolfrum and D. Turaev. Instabilities of lasers with moderately delayed optical feedback. Opt. Comm., 212(1-3):127-138, 2002.

[95] H.-J. Wünsche. Equivalence of the LK and TWE approach in the weak feedback limit. unpublished. 
[96] H.-J. Wünsche, O. Brox, M. Radziunas, and F. Henneberger. Excitability of a Semiconductor Laser by a Two-Mode Homoclinic Bifurcation. Phys. Rev. Lett., 88:023901, 2002.

[97] H.-J. Wünsche, M. Radziunas, S. Bauer, O. Brox, and B. Sartorius. Modeling of Mode Control and Noise in Self-Pulsating PhaseCOMB Lasers. IEEE J. on Sel. Top. Quantum Electron., 9(3):857-864, 2003. 


\section{Lebenslauf}

Name

Nationalität

Familienstand

09/1979-07/1983

08/1983-06/1992

07/1992-06/1993

\section{Universität}

11/1993-02/1999 Studium der Physik an der Universität Regensburg.

Diplomarbeit: "In situ Wachstumsanalyse von MBE-SchichtSystemen mit RDS und RHEED", am Lehrstuhl Prof. Dr. W. Gebhardt.

\section{Berufserfahrung}

04/1999-

Stefan Bauer

geboren am 24. Januar 1973 in Vilshofen

deutsch

verheiratet

Schule

Grundschule Ortenburg

Gymnasium Vilshofen

Wehrdienst

Wissenschaftlicher Mitarbeiter am Fraunhofer-Institut

für Nachrichtentechnik, Berlin.

Arbeitsgebiete: Entwicklung und Charakterisierung von

Mehrsektions-Halbleiterlasern, optische Nachrichtentechnik,

3R-Regeneration. 



\section{Danksagung}

Diese Arbeit wurde erst durch die Hilfe vieler Kollegen möglich. Es ist mir eine Freude, allen zu danken, deren Unterstützung zum Gelingen beigetragen hat. Mein besonderer Dank gilt ...

...Prof. Dr. Fritz Henneberger für die Bereitschaft, diese externe Promotion zu betreuen.

... Dr. Hans-Jürgen Wünsche als unermüdlicher und stets hilfsbereiter Diskussionspartner, zu Tages- und zu Nachtzeiten. Seine Ideen und die fachliche Hilfe haben massgeblich zum Gelingen beigetragen.

... Prof. Dr. Oliver Benson und Prof. Dr. Wolfgang Elsäßer für die Gutachten.

... Dr. Bernd Sartorius und Dr. Hans-Peter Nolting, die den Freiraum für diese Arbeit gewährten, um sie neben den Projektverpflichtungen durchzuführen.

... Alcatel Corporate Research Center als Industriepartner.

... Olaf Brox und Dr. Detlef Hoffmann als Kollegen, die immer mit Rat und Tat zur Seite standen und auch abwegigen Ideen gegenüber aufgeschlossen waren.

...Dr. Jochen Kreissl, der unzählige Wafer realisierte. Durch ihn wurden Ideen in kürzester Zeit zu funktionsfähigen Lasern.

... Matthias Biletzke für seine Hilfe in allen praktischen Belangen.

... Dr. Jan Sieber und Dr. Matthias Wolfrum, die mit ihren mathematischen Modellen

der Laserphysik zu Leibe rückten und so ein tieferes Verständnis der experimentellen Ergebnisse ermöglichten.

... Dr. Mindaugas Radziunas für Simulationen und das Programm LDSL.

...den Teilnehmern des Seminars Mathematische Photonik, insbesondere Dr. Klaus Schneider, Dr. Lutz Recke, Dr. Uwe Bandelow und Dr. Hans Wenzel. Die Diskussionen im Seminar und in den obligatorischen Nachsitzungen waren vielfach Anregung zu weiteren Untersuchungen.

... Oleg Ushakov und Nikolay Korneyev, die an der HU Arbeiten durchführten, für die am HHI keine Zeit blieb.

... allen Bewohnern der dritten Etage.

... Carsten Bornholdt für die Versorgung mit der jeweils neuesten Generation an Messgeräten.

... allen übrigen Kollegen im Projektteam, Dr. Christiana Bobbert, Gero Bramann, Prof. Dr. Ulrike Busolt, Hao Ding, Dr. Martin Möhrle, Mathias Mönster, PierreOlivier Ntongmo, Jeremie Renaudier, Gabriel Sahin, Juraj Slovak und Dr. Tolga Tekin. ... meiner Familie für die langjährige Unterstützung, insbesondere meiner Frau Birgit. 



\section{Selbständigkeitserklärung}

Hiermit erkläre ich, die vorliegende Arbeit selbständig ohne fremde Hilfe verfasst und nur die angegebene Literatur und Hilfsmittel verwendet zu haben.

Stefan Bauer 